\title{
The somatosensory system: Exploration of digit-area somatotopy and feature-based attention
}

\section{Dissertation}

in partial fulfillment of the requirements for the degree

"Doctor rerum naturalium (Dr. rer. nat.)"

in the Neuroscience Program (Faculty of Biology) at Georg-August-Universität Göttingen

submitted by

Meike Annika Schweisfurth

from Langenfeld (Rheinland)

Göttingen, 2013 

Doctoral thesis committee: Prof. Dr. Jens Frahm (First referee)

Biomedizinische NMR Forschungs $\mathrm{GmbH}$

Max-Planck-Institut für biophysikalische Chemie

Am Faßberg 11

37077 Göttingen

Prof. Dr. Stefan Treue (Second referee)

Cognitive Neuroscience Laboratory

Deutsches Primatenzentrum

Kellnerweg 4

37077 Göttingen

Prof. Dr. Christiane Thiel

Carl von Ossietzky Universität Oldenburg

Fak. V, Institut für Psychologie

AG Biologische Psychologie

26111 Oldenburg

Dr. Renate Schweizer (Advisor)

Biomedizinische NMR Forschungs $\mathrm{GmbH}$

Max-Planck-Institut für biophysikalische Chemie

Am Faßberg 11

37077 Göttingen

External examiners:

PD Dr. Peter Dechent

MR-Forschung in der Neurologie und Psychiatrie Universitätsmedizin Göttingen

Robert-Koch-Str. 40

37075 Göttingen

Prof. Dr. Alexander Gail

Sensorimotor Group

Deutsches Primatenzentrum

Kellnerweg 4

37077 Göttingen

Dr. Igor Kagan

Decision and Awareness Group

Deutsches Primatenzentrum

Kellnerweg 4

37077 Göttingen

Date of the oral examination: $10^{\text {th }}$ of June, 2013 

Herewith I declare that I have written this thesis independently and with no other aids and sources than quoted.

Göttingen, $8^{\text {th }}$ of May 2013

Meike Schweisfurth 

To my family 


\section{Acknowledgements}

First of all, I am greatly thankful to Jens Frahm and Stefan Treue for giving me the opportunity to work in their laboratories, letting me not only do research under excellent laboratory conditions but also profit from their outstanding mentorship, experience, knowledge, and advice. In this context, I would also like to deeply thank Renate Schweizer for being a very committed and enthusiastic advisor and mentor, who was always there for advice, suggestions, and encouragement.

My thanks also go to Christiane Thiel from Oldenburg for helpful questions and suggestions during progress committee meetings. Also, I am grateful to Steffen Katzner from Tübingen for support with and partial programming of the LATER analysis and helpful comments concerning the psychophysics manuscript.

I would also like to thank all my colleagues. At the MPI, Tibor Auer helped me upon FSL problems, Sylke Wallbrecht in administrative issues, and Kurt Böhm by "re-animating" my overloaded computer. Having spent most of my working hours at the MPI, I would like to thank my colleagues there who really made work a fun place - with table soccer, lunch, soccer, walks, break entertainment, and mostly with helpful and joyful discussions. At the DPZ, I would like to thank Ralf Brockhausen for programming my MWorks plugins, Klaus Heisig for the foot-pedal build-up, Vera Marks for introducing me into MWorks, and Beatrix Glaser and Karin Peinemann for administrative help. Although having worked less at the DPZ, I am also grateful to my colleagues there, who always warmly integrated me in discussions and many fun events; especially the retreat has been a great scientific and group experience.

My thanks also go to the ones who made it possible for me to visit exciting conferences and courses in attractive countries as Spain, Canada, China, and the USA during my PhD education. For a great time during these conferences, my thanks mainly go to Renate Schweizer, Tibor Auer, Peter Dechent, Carsten SchmidtSamoa, and Sonia Baloni.

I would also like to thank the Neurosenses program for financial and educational support during my dissertation. My thanks also go to the International Max Planck Research School for Neurosciences (Michael Hoerner and Sandra 
Drube), not only for their financial and administrative support during my master and $\mathrm{PhD}$ studies but also for giving me as trained mathematician the chance to switch to the fascinating field of neuroscience. In this context, I would also like to thank Fred Wolf and my family for the encouragement to take this step.

I am also thankful to the five practical students (Philipp Ulbrich, Annika Graß, Kirsten Emmert, Julio Santos Viotti, and Julian Geiger) that I supervised during the last three years. They not only gave me the chance to develop and improve my teaching skills but also helped me with excellent questions and fruitful discussions.

I would also like to thank Irene Böttcher-Gajewski who took professional pictures of my equipment used for the paper and this thesis.

Not to forget, I am also grateful to my subjects, who participated in my experiment with never-ending patience and engagement.

Also, I am very thankful to Renate Schweizer, Sabine Hofer, Tim Schweisfurth, and Cliodhna Quigley for proofreading parts of this thesis and supporting me with helpful comments.

I would like to thank my personal environment that greatly helped me in finding a healthy work-life balance. I am greatly thankful to my amazing friends who helped me with both their strong encouragement and understanding for my work but also with great distraction from my work. For the latter, I also would like to thank all those who made my choirs Unicante and Procant not only possible but also a fun experience.

My final and warmest thanks go to my family, mainly to my parents and my brother Tim. I would like to thank them for their complete understanding, their strong encouragement, and their everlasting love. Thank you for everything! 



\section{Contents}

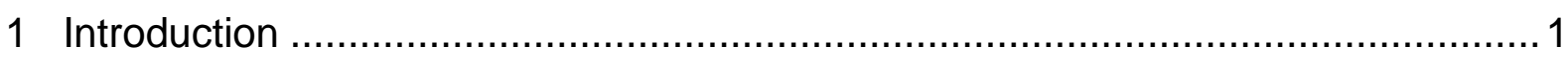

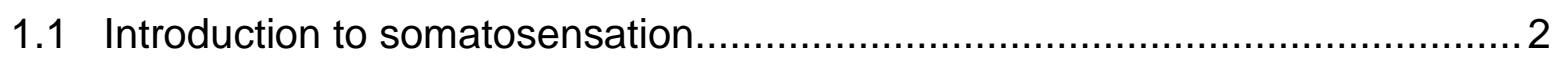

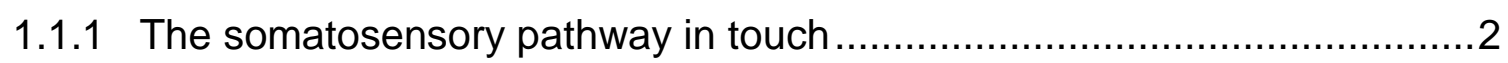

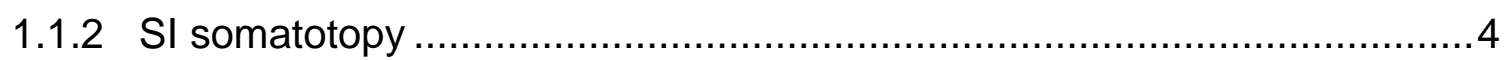

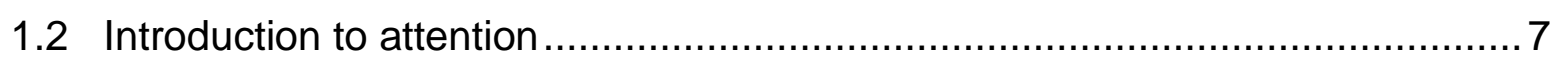

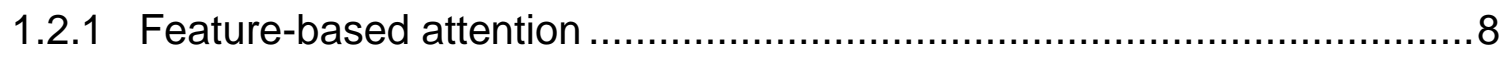

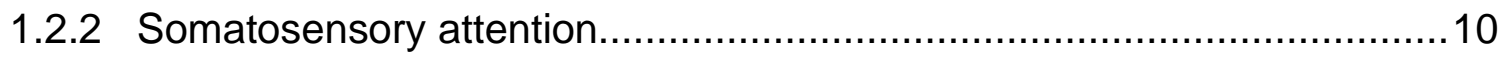

1.3 Summary of the topics of this thesis ..................................................... 12

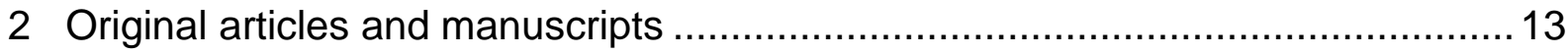

2.1 Functional MRI indicates consistent intra-digit topographic maps in the little but not the index finger within the human primary somatosensory cortex ............. 15

2.2 Functional MRI reveals individual variations in the complete map of human digit phalanges in the primary somatosensory cortex.......................................23

2.3 Feature-based attentional modulation of orientation perception in

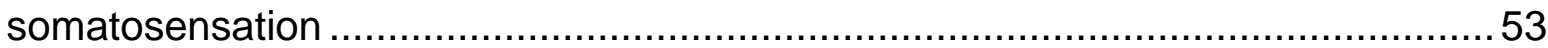

2.4 Exploration of feature-based somatosensory modulation of responses to

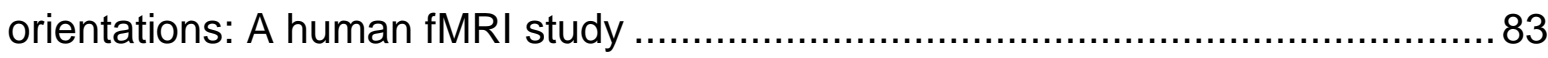

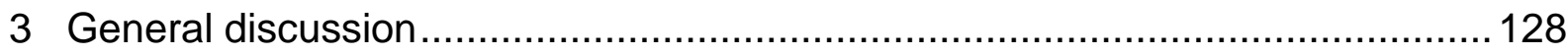

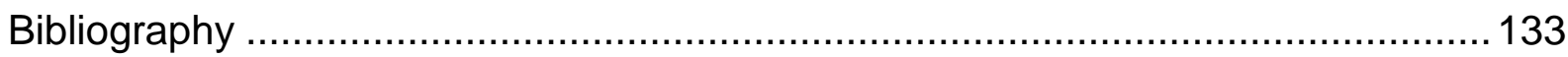

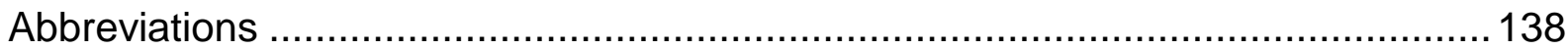

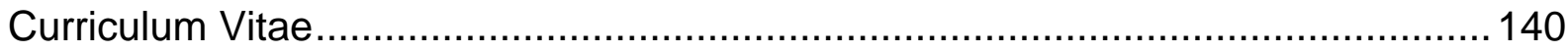





\section{Introduction}

Even though the skin is by far the largest sensory organ that we humans possess and immensely important for coping with everyday life, the somatosensory system has been studied far less than the visual or auditory system. Hence, many basic and important questions still remain to be explored. This thesis is devoted to the exploration of several features of the human somatosensory system. With behavioral and systemic tools, light is shed into basic questions of touch processing, thereby contributing to the presently comparatively scarce body of knowledge about somatosensation.

This thesis comprises four studies, focusing on two different aspects of the human sense of touch. The first two studies investigate whether and how the peripheral digit-area topography is reflected in the cortical digit and phalanx representations, exploring the presence of across-digit and intra-digit somatotopy and characterizing the individual human all-phalanx digit map in unprecedented detail. Studies three and four explore the existence of global feature-based attention in the somatosensory domain, for the first time assessing the issue both with behavioral tools and functional imaging.

In this section, a general introduction into the somatosensory system and relevant attentional mechanisms is given, focusing on feature-based attention and any kind of somatosensory attention. The second section forms the main part of this thesis, containing one original article, one submitted, and two prepared manuscripts. In the third section, the findings of this thesis are shortly summarized and put into a more general context. 


\subsection{Introduction to somatosensation}

The somatosensory system is special among the sensory systems (for a review, see Gardner, 2010). Firstly, its receptors are not densely localized at one or two body sites as in vision, audition, olfaction, and gustation, but spread across the whole body. Via external (at the body surface) and internal (within our body) somatosensory receptors, we are provided with information both about our external (environment) and internal (body) world. Second, the somatosensory system can be further subdivided into four different modalities, which together can evoke the most pleasant but also the most unpleasant human sensations. Proprioception exploits information from internal and external stretch receptors to keep track of body posture and extremity position. Thermoreceptors within the skin allow for detection of temperature changes. The sensation of pain is caused by noxious stimuli to nociceptors, free nerve endings in the skin. Finally, the sense of touch allows us via mechanoreceptors to describe the properties of objects standing in direct contact with our skin -another speciality of the somatosensory compared to other sensory systems. This thesis is entirely devoted to this somatosensory modality - to touch.

\subsubsection{The somatosensory pathway in touch}

External tactile information enters the body via mechanoreceptors in the skin. Via different architectures, these mechanoreceptors can be further subdivided by their receptive-field sizes and by their response properties to long-lasting stimuli. Whereas the larger Ruffini's endings and Pacinian corpuscles lie deep within the dermis, the smaller Merkel's disks and Meissner's corpuscles are located at the border between epidermis and dermis or just below. The high spatial acuity for discrimination of stimuli mainly results from these smaller receptors (Johansson and Vallbo, 1978). While the slowly-adapting Merkel's disks and Ruffini's endings generate a sustained response to a static stimulus, the fast-adapting Meissner's and Pacinian corpuscles rather respond to vibratory than static stimuli; low-frequency $(2-40 \mathrm{~Hz})$ information is primarily encoded by Meissner's, high-frequency information $(60-400 \mathrm{~Hz})$ by Pacinian corpuscles (for an overview, see Gardner et al., 2000).

Tactile information is mediated from the skin to the cortex via the dorsal column - medial lemniscal pathway. Each skin mechanoreceptor incorporates 
unmyelinated axonal branches with mechanosensitive ion channels. These primary afferent axons (type $A B$ ) of dorsal root ganglion cells enter the spinal cord via the dorsal root and ascend via the dorsal column to the dorsal column nuclei between spinal cord and medulla. Here, the first synapse of the pathway is located. Then, the consecutive axons decussate and ascend the medial lemniscus until they synapse onto neurons in the ventral posterior nucleus of the thalamus. These then project to Brodmann area (BA) $3 \mathrm{~b}$ and 1 of the (with respect to the sensory stimulus) contralateral primary somatosensory cortex (SI, Jones and Friedman, 1982; Gardner, 1988), located at the postcentral gyrus, posterior to the central sulcus (for an overview, see Gardner et al., 2000).

The primary somatosensory cortex consists of BA $3 a, 3 b, 1$, and 2, arranged in parallel along the anterior-to-posterior axis of the postcentral gyrus (see Fig. 1). Generally, BA $3 a$ is assumed to lie deep within the fundus of the central sulcus, $\mathrm{BA} 3 \mathrm{~b}$ at the anterior bank, BA 1 at the crown, and BA 2 at the posterior bank of the postcentral gyrus (Brodmann, 1909; Vogt and Vogt, 1919; Geyer et al., 2000). However, more recent cytology of post-mortem human brains revealed a high variability in the relative location and extent of Sl's Brodmann areas along the

A The somatosensory cortex

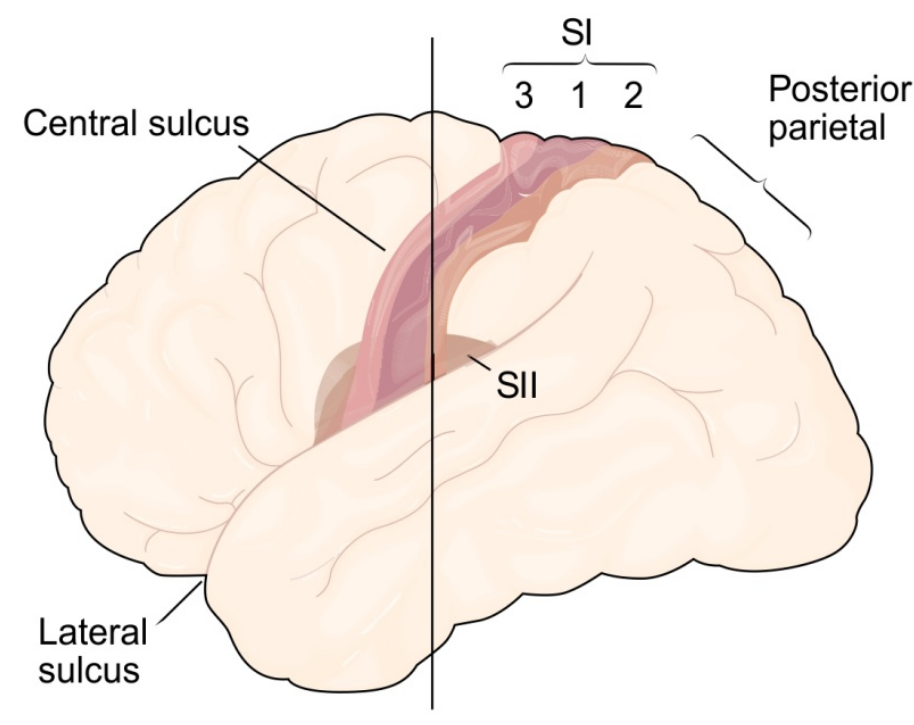

B Coronal section through SI and SII

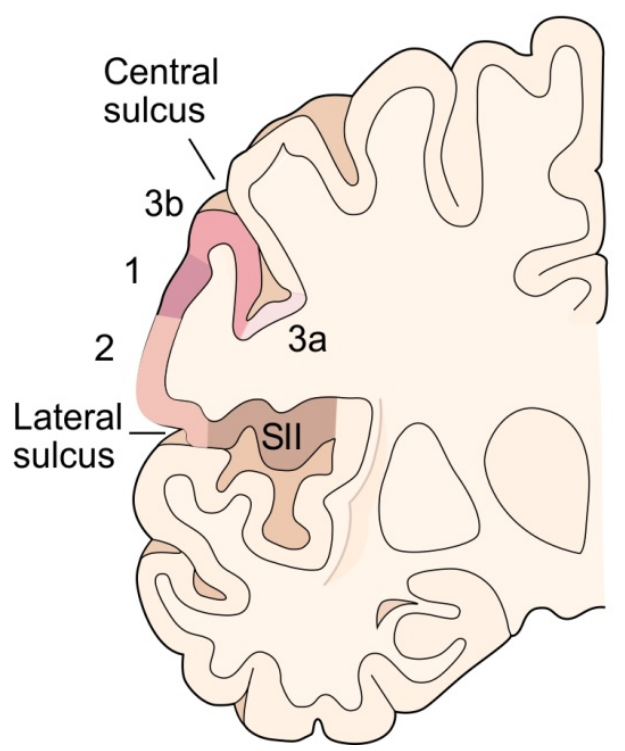

Figure 1. Somatosensory areas of the cerebral cortex. (A) Lateral view of the brain, illustrating the primary (SI) and secondary (SII) somatosensory cortex. (B) Coronal section through the postcentral gyrus, showing the cytoarchitectonically different Brodmann areas $3 \mathrm{a}, 3 \mathrm{~b}, 1$, and 1 of $\mathrm{SI}$ as well as their positions relative to SII. Adapted from Figure 23-10 (Gardner and Johnson, 2012). 
postcentral gyrus (Geyer et al., 1999). Because an in-vivo method for distinguishing the four Brodmann areas in individual subjects is still missing, current functional imaging studies still use the above description for region-of-interest definition. The different BAs in $\mathrm{SI}$ are involved in different functional tasks. Whereas activation in BA $3 a$ is only evoked by proprioceptive information, the other areas all respond to tactile information (with BA 2 responding to both touch and proprioception). BA 1 and 2 receive strong input from $B A 3 b$ and are assumed to be involved in higherorder processing (Gardner, 1988; Garraghty et al., 1990; Eskenasy and Clarke, 2000). Receptive fields tend to increase in size and complexity from the more anterior to the more posterior Brodmann areas (Sur et al., 1980; Gardner, 1988). From SI, the information is passed on via two different pathways (for a review, see Gardner and Johnson, 2010): The ventral pathway projects to the second somatosensory cortex (SII), located in the parietal operculum, and is important in tactile object recognition. The dorsal one incorporates the motor cortex as well as posterior parietal areas (BA 5, 7, 39, 40) and is involved in movement-guiding sensorimotor planning.

\subsubsection{SI somatotopy}

As this thesis mainly focuses on the primary somatosensory cortex, this paragraph will provide more detail about its functional organization.

In SI, the sense of touch is represented in a somatotopic way, meaning that areas next to each other on the skin are also represented next to each other at the cortex. Because different humans possess a very similar body topology, the arrangement of body-part representations in SI is very consistent across subjects. This could be shown by Penfield and coworkers in neurosurgical operations on epilepsy patients (see Fig. 2; Penfield and Boldrey, 1937; Penfield and Rasmussen, 1950). However, the map has some discontinuities; the upper proximity, for example, is represented between face and head. Areas of high importance for touch sensation and hence high mechanoreceptor density, as the fingers and the lips, present with an over-proportionally large cortex representation. By combining these features, the SI somatotopy is often described by Penfield's so-called "Sensory homunculus", the "little man" in our somatosensory cortex. In later electrophysiological recordings in several monkey species, this somatotopic representation was confirmed (Merzenich 
Figure 2. Penfield's somatosensory homunculus. This schematic of Brodmann area 1 of the primary somatosensory cortexreflects the cortical neighborhood relationships between body parts. Those parts of the body that are crucial for tactile discrimination (as the lips and the digits) have a higher peripheral receptor density and overproportionally large cortical representations in $\mathrm{SI}$, as visualized in this schematic. Figure 18-10 (Amaral, 2000).

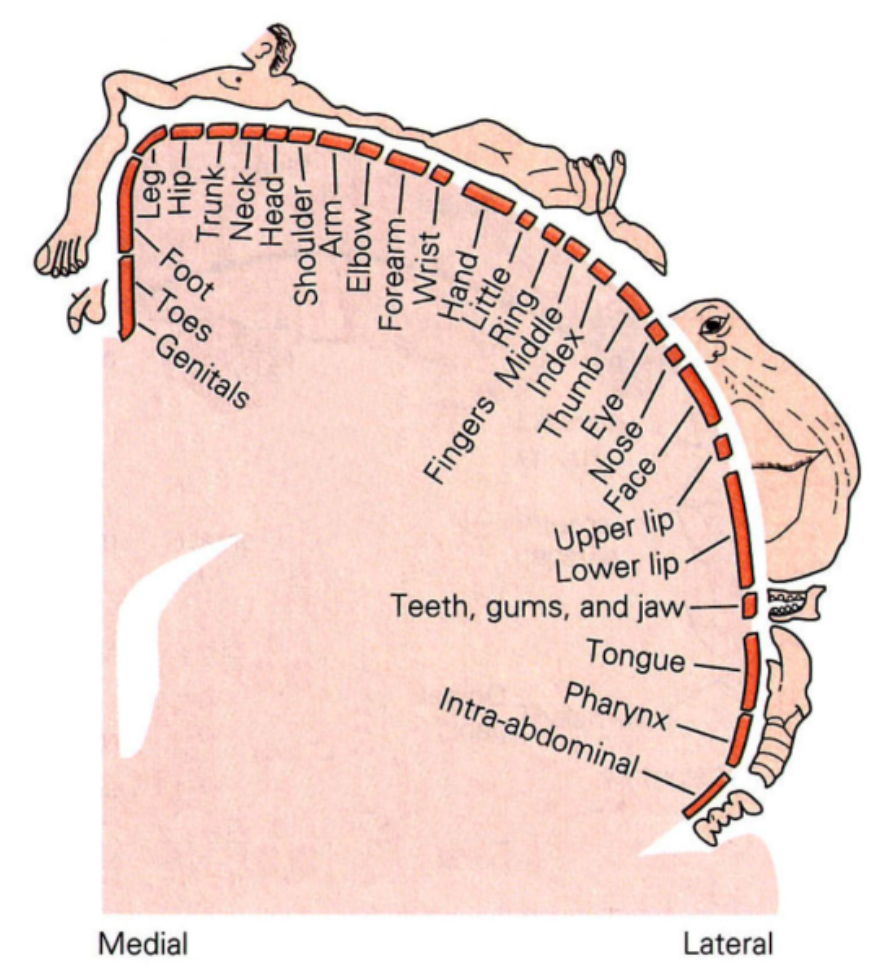

et al., 1978; Kaas et al., 1979; Nelson et al., 1980). These monkey studies, however, did not only observe a single sensory representation in SI but found at least two, one in BA $3 b$ and one in BA 1.

For the digits, Penfield and colleagues observed a medial-to-lateral succession of the human cortical digit representations from little finger (D5) to thumb (D1) (see Fig. 3; Penfield and Boldrey, 1937; Penfield and Rasmussen, 1950). This pattern has been corroborated in human imaging studies mapping the five fingertips (e.g. Nelson et al., 2008; Schweizer et al., 2008; Sanchez-Panchuelo et al., 2010; Martuzzi et al., 2012), some of these observing it in BA 3b, 1, and 2,

Figure 3. Schematic illustration of the SI across-digit somatotopy. Within SI, the digits are generally shown to be represented in ordered medial-to-lateral succession from D5 to D1. Adapted from Fig. 23-7 A (Gardner and Kandel, 2000).

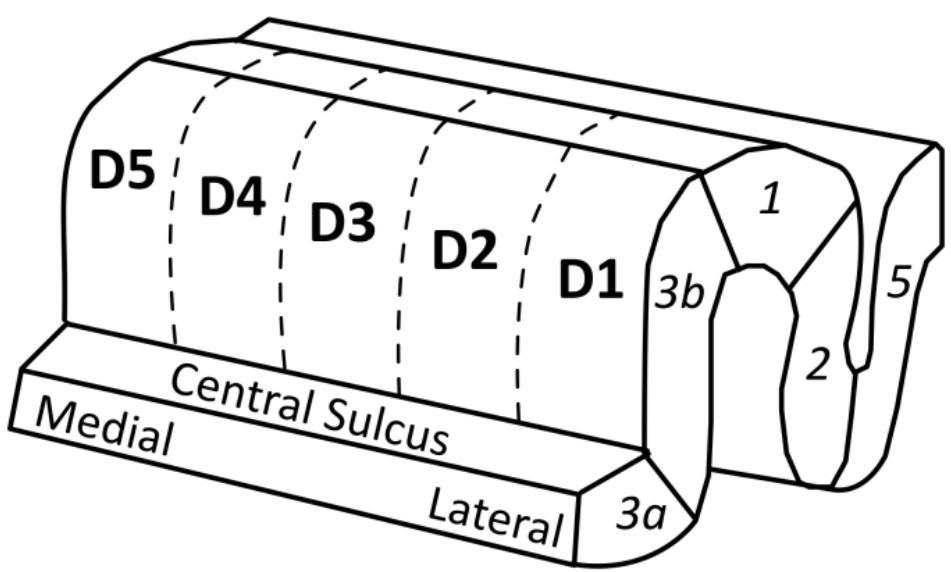


with increasing overlap and decreasing clarity from the former to the latter area (Martuzzi et al., 2012). Electrophysiological non-human primate studies (Merzenich et al., 1978; Kaas et al., 1979; Nelson et al., 1980; Iwamura et al., 1983a,b) also found this digit succession, observing it both in BA $3 b$ and 1.

By mapping the phalanges within each digit, several (Merzenich et al., 1978; Kaas et al., 1979; Nelson et al., 1980) but not all (Iwamura et al., 1983a,b) of these monkey studies further found rostral-to-caudally ordered within-digit representations from the fingertip ( $p 1)$ to the proximal phalanx (p3) in BA $3 b$ and mirror-reversed in BA 1. In humans, the existence of such an intra-digit somatotopy is still under debate (see e.g. Blankenburg et al., 2003; Overduin and Servos, 2004; Schweisfurth et al., 2011; Sanchez-Panchuelo et al., 2012). The first two chapters of this thesis are devoted to further exploration of this issue. 


\subsection{Introduction to attention}

In every moment in life, our sensory systems are bombarded with a tremendous amount of stimuli from the external world. Due to energetic restrictions of our brain, only a tiny amount of this incoming information can be processed up to awareness. In order to successfully perform in everyday life, the behaviorally most relevant information should be selected. Attention is thought to be the most important mechanism for filtering this information out of the gigantic amount of input.

At least two very different influences define which sensory signals are selected as behaviorally relevant. Incoming sensory signals contribute by bottom-up aspects (for a review, see Mulckhuyse and Theeuwes, 2010) through automatic allocation of attention to high-contrast stimuli, realized by the hard-wired center surround structure of receptive fields of sensory-cortex neurons. On the other hand, top-down influences like voluntary attention play a crucial role in tagging a stimulus as relevant. This mechanism allows for the intriguing possibility to voluntarily influence the processing in our sensory cortices, solely based on our current state of mind, reached via prior experiences and future expectancies concerning our internal and external world. Combining sensory-driven bottom-up and voluntary top-down attention, an integrated, sparse saliency map is computed, strongly highlighting the stimuli of presumed behavioral importance (for a review, see Treue, 2003).

Both bottom-up (or exogenous) and top-down (or endogenous) attention have been observed in numerous studies and with different techniques, mostly for the visual and auditory modality. In psychophysical studies, attentional effects are usually reflected by an increase in accuracy or a decrease in reaction time (e.g. Posner et al., 1978). In functional magnetic resonance imaging (fMRI) studies, a higher signal is observed for attended versus unattended stimuli (e.g. JohansenBerg et al., 2000). Electrophysiologically, directing attention into a cell's receptive field (spatial attention) modulates the firing rate of the neuron; generally, attention to the cell's preferred stimulus increases it, whereas attention to the unpreferred stimulus decreases it (feature-based attention; for a review, see Treue, 2001).

Although attentional modulation is already found as early as in the primary visual cortex, attentional influences are generally assumed to increase along the processing hierarchy in the visual system (for a summary, see Maunsell and Cook, 2002). 
Attention can be directed to very different targets. The most studied attentional phenomenon is spatial attention where attention is directed to the location of a stimulus. It is usually described as an attentional spotlight, leading to increased sensitivity at an attended compared to unattended location, allowing us to better process information entering from that location, at the expense of stimuli at other locations. The second attentional category is object-based attention where attention is directed to an entire object (for example a face), which leads to more efficient processing of attended as well as unattended features of that object (O'Craven et al., 1999). As third category, attention can be feature-based, as described in more detail in the next paragraph. This thesis is devoted to the exploration of feature-based attention.

\subsubsection{Feature-based attention}

In feature-based attention (for a review, see Treue, 2007), a certain feature (e.g. vertical) out of a certain stimulus dimension (e.g. orientation) is chosen as behaviorally-relevant and hence attended (see Fig. 4). Imagine an animal in the jungle, not willing to end as a tiger's prey. For this animal it makes sense to constantly watch out for vertical stripes to be able to instantly flee upon arrival of a tiger. Closer to our own everyday life, a friend will be easier to find in a crowd, if we

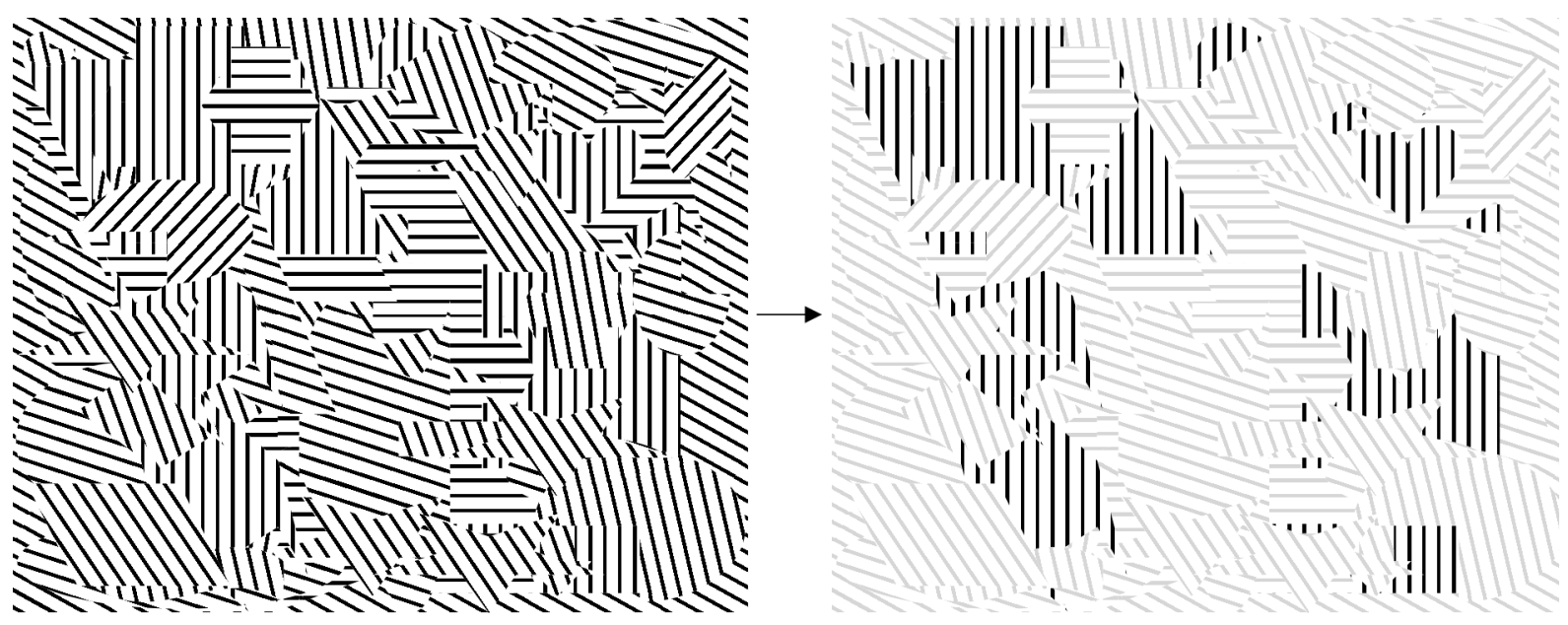

Figure 4. Abstract illustration of the effect of feature-based attention. Searching for a heart in a graphic crowded with orientations is a difficult task. Knowing that the heart is of vertical orientation, all objects with that feature are attended and hence more efficiently processed, allowing for localization of the heart at the upper right. 
for example know the color of his jacket. The effectiveness of visual feature-based attention can be impressively demonstrated by inattentive blindness, where subjects watching a video are unable to perceive a black gorilla entering the scene for several seconds, purely due to attending only to the feature white (Simons and Chabris, 1999). This phenomenon also highlights the drawbacks of attentional selection, letting us miss potentially relevant objects or events.

For the visual modality, research in the last two decades found evidence for feature-based attention with several different techniques, including human psychophysics and functional imaging as well as monkey electrophysiology. The strongest cortical effects of feature-based attention have been reported in those visual areas that are specialized for processing of the attended stimulus dimension (Saenz et al., 2002; Stoppel et al., 2011).

Feature-based attention in vision was found to be a global phenomenon. In many studies of feature-based attention, simultaneous spatial attention was directed to a well-defined location in space. Crucially, feature-based attention did not only lead to better processing of the attended feature at this attended location; its effects could even be observed at unattended locations as distant as the opposite hemifield. Behaviorally, this would allow an animal to rapidly and sensitively detect a tiger on the prowl even if it approaches from an unexpected direction. This global effect of attention was first shown using monkey electrophysiology (Treue and MartinezTrujillo, 1999). Here, attention to a cell's preferred feature at a distant location led to higher firing rates of the neuron compared to when the unpreferred feature was attended at the same distant location. These findings are well described by the feature-similarity gain model (Treue and Martinez-Trujillo, 1999), stating that featurebased attention modulates the firing rate of a neuron in a multiplicative way, with the sign as well as the magnitude of the attentional gain modulation depending on the similarity between the currently attended feature and the neuron's own feature preference. On the population level, however, non-multiplicative modulation can be observed (Martinez-Trujillo and Treue, 2004). Global feature-based attention was reported also in humans by fMRI (Saenz et al., 2002; Serences et al., 2006) and psychophysical studies (Rossi and Paradiso, 1995; Saenz et al., 2003). 


\subsubsection{Somatosensory attention}

As this dissertation focuses on the exploration of the tactile modality, in this paragraph an overview on the knowledge on tactile attention is given. So far, most research in somatosensory attention has been devoted to spatial attention and to the effects of attending versus ignoring a stimulus. In contrast to the visual modality, far more questions are still under debate or not even investigated.

The question whether orienting attention to a tactile stimulus leads to a higher cortical signal compared to ignoring the same stimulus has been the focus of many tactile-attentional imaging studies. In SII, attentional effects have repetitively been shown by $\mathrm{FMRI}$, positron emission tomography (PET), and magnetoencephalography (MEG) (Johansen-Berg et al., 2000; Hämäläinen et al., 2000; Nelson et al., 2004; Sterr et al., 2007; Burton et al., 2008). The existence of attentional effects as early as in $\mathrm{SI}$ is supported by most but not all imaging studies (for a discussion on that issue, see Johansen-Berg et al., 2000). Electrophysiological non-human primate studies corroborate the existence of attentional effects both for SI and SII (Hsiao et al., 1993; Hyvärinen et al., 1980). However, the evidence for hierarchical augmentation of attentional effects is sparser and less clear in the somatosensory compared to the visual system (for a review, see Johansen-Berg, 2000).

Spatial tactile attention has been extensively explored in psychophysical studies, mostly exploiting variants of the well-established Posner paradigm (Posner, 1978). In this paradigm, subjects have to detect or discriminate targets at one out of (at least) two locations, one of which is far more probable than the other, as indicated by a previously presented cue. If faster reaction times or higher accuracy rates (depending on the exact experimental setting) are then observed for the cued compared to the uncued location, this effect is generally interpreted as spatial attention (e.g. Posner, 1978; Posner, 1980). For tactile discrimination tasks, spatial attention was revealed by numerous studies reporting faster reaction times (Posner, 1978; Spence et al., 2000; Forster and Eimer, 2005; Chica et al., 2007; Van Ede et al., 2012) or higher accuracy rates (Sathian and Burton, 1991; Van Ede et al., 2012) for targets at the cued compared to the uncued location, well in accordance with visual findings. Looking at the cortical aspects of spatial attention, event-related potential (ERP) studies could show modulation of early somatosensory components 
upon attending to one hand versus the other (e.g. Desmedt and Robertson, 1977). Such modulation was also found upon attending to one finger versus another within the same hand, and- to a lower degree- even upon attending to one phalanx versus another within the same finger (Eimer and Forster, 2003), giving evidence for a gradient of tactile spatial attention. Along similar lines, it could be shown that the somatotopic SI digit map can be modulated purely by changes in the attentional state (e.g. Noppeney et al., 1999; Buchner et al., 2000; Braun et al., 2002), e.g. with attention to individual fingers leading to a larger inter-digit distance compared to joint attention to all fingers (by MEG, Braun et al., 2002).

Non-spatial attributes of attention have only very rarely been explored for the tactile domain. Recent ERP studies (Gillmeister et al., 2009, 2013) found evidence for object-based attention in touch. Selective attention to certain stimulus dimensions like orientation or frequency was also explored in several studies (Hoechstetter et al., 2000; Burton et al., 1999, 2008; Sinclair et al. 2000), which revealed decreased reaction times for valid compared to neutral cueing of the target's stimulus dimension (Burton et al., 1999; Sinclair et al. 2000), but did not find any cortical effects of attention (Burton et al., 1999, 2008). In strong contrast to the visual modality, feature-based attention (i.e. the effect of focusing on a well-defined feature (e.g. vertical) within a chosen stimulus dimension (e.g. orientation)) has so far only been tackled by one pure ERP study by Forster and Eimer (2004), giving a first hint to a global mechanism of feature-based attention similar as observed in vision.

Tactile feature-based attention plays an important role in everyday life. Imagine that you search for your key in your bag, which often has to be done in complete darkness; attending to key-like riffles or to metal-like sensations then should help to find the key. In the darkness, an immobile object can only be determined by its continuous features along with its shape; for lighter objects, also proprioceptive information can be used for object grouping (Gillmeister et al., 2010). The third and fourth study of this thesis explore feature-based attention in touch. 


\subsection{Summary of the topics of this thesis}

This thesis explores two different aspects of the somatosensory system: Digitarea somatotopy and tactile feature-based attention.

The first two reported studies focus on digit-area somatotopy. While the presence of a medial-to-lateral succession of the fingertip representation in the primary somatosensory cortex has been confirmed in humans and many non-human primate species, there is still an on-going debate about the existence of an ordered and consistent intra-digit somatotopy across human subjects. The first two studies reported in this dissertation explore this issue. Whereas the first study focuses on the relative position between tip and base representations of index and little finger, the second study provides the first complete mapping of the SI digit area of individual subjects and hence addresses the issues of both intra-digit and across-digit somatotopy.

The third and fourth study of this dissertation explore feature-based attention in the somatosensory system, which has hardly been addressed in the literature. The third study reported in this thesis to our knowledge provides the first investigation of behavioral correlates of feature-based attention in touch, while the fourth study is the first to employ functional MRI to address the issue of tactile feature-based attention. 


\section{Original articles and manuscripts}

This chapter contains the following research articles and manuscripts:

\subsection{Functional MRI indicates consistent intra-digit topographic maps in the} little but not the index finger within the human primary somatosensory cortex Schweisfurth MA, Schweizer R, Frahm J (2011) Neurolmage 56: 2138-2143 Author contribution: SM, SR, and FJ designed the experiment. SM implemented the experiment and collected and analyzed the data. SM and SR wrote the manuscript; FJ edited the manuscript.

\subsection{Functional MRI reveals individual variations in the complete map of} human digit phalanges in the primary somatosensory cortex Schweisfurth MA, Frahm J, Schweizer R (prepared for submission) Author contribution: SM and SR designed the experiment. SM implemented the experiment, collected and analyzed the data. SM and SR wrote the manuscript; FJ edited the manuscript.

\subsection{Feature-based attentional modulation of orientation perception in somatosensation}

Schweisfurth MA, Katzner S, Schweizer R, Treue S (prepared for submission) Author contribution: TS, SM, and SR designed the experiment. SM implemented the experiment and collected and analyzed the data. SM and KS implemented the LATER analysis. SM wrote the manuscript; KS and TS edited the manuscript.

\subsection{Exploration of feature-based somatosensory modulation of responses to orientations: A human fMRI study \\ Schweisfurth MA, Treue S, Frahm J, Schweizer R (prepared for submission) Author contribution: SM, TS, and SR designed the experiment. SM implemented the experiment and collected and analyzed the data. SM wrote the manuscript; SR and FJ edited the manuscript.}





\subsection{Functional MRI indicates consistent intra-digit topographic maps in the little but not the index finger within the human primary somatosensory cortex}

In this chapter, BA $3 \mathrm{~b}$ intra-digit topography was explored solely for the tips and bases of index and little finger, allowing for a high number of averages per stimulation-site condition. Interestingly, intra-digit topographic maps consistent across subjects were found for the little but not the index finger. This different level of across-subjects consistency might be related to the more individual use of the index compared to the little finger in everyday life. The chapter is included as published in Neurolmage. 


\title{
Functional MRI indicates consistent intra-digit topographic maps in the little but not the index finger within the human primary somatosensory cortex ${ }^{i}$
}

\author{
Meike A. Schweisfurth ${ }^{\mathrm{a}, \mathrm{b}, *}$, Renate Schweizer ${ }^{\mathrm{a}}$, Jens Frahm ${ }^{\mathrm{a}}$ \\ a Biomedizinische NMR Forschungs GmbH am Max-Planck-Institut für biophysikalische Chemie, 37070 Göttingen, Germany \\ ${ }^{\mathrm{b}}$ Cognitive Neuroscience Laboratory, German Primate Center, Kellnerweg 4, 37077 Göttingen, Germany
}

\section{A R T I C L E I N F O}

\section{Article history:}

Received 12 January 2011

Revised 10 March 2011

Accepted 14 March 2011

Available online 21 March 2011

\section{Keywords:}

fMRI

Human

Index finger

Intra-digit somatotopy

Little finger

\begin{abstract}
A B S T R A C T
This study explored the question of intra-digit somatotopy of sensory representations in the little and index finger of 10 subjects using tactile stimulation of the fingertip (p1) and base (p4) and functional magnetic resonance imaging (fMRI) at $1.5 \mathrm{~mm}$ isotropic spatial resolution. The Euclidian distances between $\mathrm{p} 1 \mathrm{and} \mathrm{p} 4$ peak representations in Brodmann area $3 \mathrm{~b}$ resulted in $5.0 \pm 0.7 \mathrm{~mm}$ for the little finger and $6.7 \pm 0.5 \mathrm{~mm}$ for the index finger. These non-collocated representations were found to be consistently ordered across subjects for the little but not the index finger. When using separate distances for medial-lateral, anterior-posterior, and inferior-superior orientations, p4 was $1.9 \pm 0.7 \mathrm{~mm}$ medial to $\mathrm{p} 1 \mathrm{for}$ the little finger in agreement with findings in macaque monkeys, whereas no consistent intra-digit somatotopy across subjects was found for the index finger. This discrepancy could point to differences in the map-forming processes based on sensory input. On the behavioral level it may be attributed to our everyday use of the hand, for which $\mathrm{p} 4$ of the index finger plays a much less important role than p4 of the little finger, which is located at the outer border of the hand.

(c) 2011 Elsevier Inc. All rights reserved.
\end{abstract}

\section{Introduction}

More than 70 years ago, Penfield and co-workers described the socalled homunculus in human primary somatosensory cortex (SI) (Penfield and Boldrey, 1937; Penfield and Rasmussen, 1950), a schematic drawing of the neuronal representation of the contralateral body surface, which in particular shows an enlarged finger area with a somatotopic mediolateral succession of the finger representations from the little finger to the thumb (d1-d5). This pattern has also been shown for non-human primates (Iwamura et al., 1983a, 1983b; Kaas et al., 1979; Paul et al., 1972) and has been reproduced in humans noninvasively by fMRI (Kurth et al., 2000; Nelson and Chen, 2008; SanchezPanchuelo et al., 2010; Schweizer et al., 2008). Cytoarchitectonically, SI consists of the anterior-to-posterior arranged Brodmann areas (BAs) 3a, 3b, 1, and 2 (Brodmann, 1909; Vogt and Vogt, 1919). For BA 3b a complete mediolateral somatotopy was reported for both the body surface of monkeys (Kaas et al., 1979; Paul et al., 1972) and the fingers of humans (Nelson and Chen, 2008; Schweizer et al., 2008).

Electrophysiological recordings revealed an intra-digit somatotopy in rostrocaudal direction in several non-human primate species, where rostral and caudal refer to the anterior and posterior direction along a flattened cortex. In anesthetized macaque (Kaas et al., 1979; Nelson

\footnotetext{
is Conflict of interest: None declared.

* Corresponding author at: Meike Schweisfurth, Biomedizinische NMR Forschungs GmbH, 37070 Göttingen, Germany. Fax: + 495112011729.

E-mail address: mschwei1@gwdg.de (M.A. Schweisfurth).
}

et al., 1980; Paul et al., 1972) and owl monkeys (Merzenich et al., 1978) the phalanx representations of a digit in BA 3b showed the fingertip (p1) in a most rostral position, followed by the second (p2) and third phalanx (p3). Despite this similarity, there is a species-specific divergence in the position of the representations that refer to the bases of the fingers, i.e. the areas of the palm beneath each finger (here denoted as p4). In owl monkeys, the volar bases are represented as part of the succession of the phalanges, caudal to the representation of $\mathrm{p} 3$ of each finger, along the border between BA 3b and BA 1 (Merzenich et al., 1978). In macaques, on the contrary, the volar bases were found lateral to the representations of d1 and d2 and medial to d5 (Nelson et al., 1980). Moreover, studies in awake monkeys showed further differences as, for example, a less pronounced intra-digit somatotopy in BA 3b (Iwamura et al., 1983a, 1983b).

Because the human postcentral gyrus is strongly folded, an intradigit somatotopy as observed in monkeys would be expected to run from inferior ( $\mathrm{p} 1$ ) to superior locations ( $\mathrm{p} 3$ or $\mathrm{p} 4$ ). The few reports of intra-digit somatotopy in human subjects in BA $3 \mathrm{~b}$ led to only limited consistency. Using magnetoencephalography (MEG) and vibrotactile stimulation of the phalanges of the index finger and palm Hashimoto and co-workers could not find statistically significant differences in location (Hashimoto et al., 1999a, 1999b). Later, Tanosaki and Hashimoto applied electric stimulation and reported $\mathrm{p} 3$ for the middle finger to be $2.3 \mathrm{~mm}$ lateral to $\mathrm{p} 1$ (Tanosaki and Hashimoto, 2004), while Hlushchuk and co-workers found that $\mathrm{p} 1$ was located $3 \mathrm{~mm}$ inferior to p3 for pneumatic stimulation (Hlushchuk et al., 2004). Using fMRI, Overduin and Servos (2004) detected intra-digit phase bands in BA 3b, 
but were not able to test for a consistent somatotopy across subjects due to limited spatial resolution. A further fMRI study used electric stimulation and again reported a pattern with p1 activation $5 \mathrm{~mm}$ inferior to $\mathrm{p} 3$ for the middle finger in individual subjects (Blankenburg et al., 2003). Interestingly, p4 was found to be located superior to p3 (in the group analysis), which would be in line with intra-digit somatotopic arrangements as in owl monkeys (Merzenich et al., 1978).

The present fMRI study further explored the question of intra-digit somatotopy at high spatial resolution. The distal phalanx and base of the index (d2) and little finger (d5) were subjected to tactile stimulation. The following questions were addressed: First, is the difference between BA $3 b$ fingertip and base representation for the index and/or little finger consistent along any direction across subjects, or in other words, is there an intra-digit somatotopic arrangement? And second, if this is not the case, is the representation of the fingertip nevertheless distinct from that of the base in individual subjects?

\section{Materials and methods}

MRI

Ten healthy subjects ( 5 women, range 18 to 30 years, mean 25 years) were recruited and determined to be right-handed according to the Edinburgh Inventory using a cut-off value of 33 (Oldfield, 1971). Before each examination informed written consent was obtained from each subject.

Subjects underwent magnetic resonance imaging (MRI) at $3 \mathrm{~T}$ (TIM Trio, Siemens Healthcare, Erlangen, Germany) using a 32-channel head coil. A sagittal T1-weighted 3D MPRAGE (magnetization-prepared rapid gradient-echo) image (repetition time $(\mathrm{TR})=2530 \mathrm{~ms}$, echo time $(\mathrm{TE})=$ $3.4 \mathrm{~ms}$, flip angle $=7^{\circ}$, acquisition matrix $=256 \times 256,160$ partitions, resolution $=1 \times 1 \times 1 \mathrm{~mm}^{3}$, total acquisition time $\left.(\mathrm{TA})=10: 49 \mathrm{~min}\right)$ was recorded and used as anatomical localizer. The motor hand knob was individually identified at the central sulcus of each subject (Yousry et al., 1997).

For functional imaging 19 double-oblique transverse-to-sagittal and transverse-to-coronal sections were positioned perpendicular to the wall of the central sulcus (cutting it in mediolateral direction) at the expected SI hand area. The sections covered the whole depth of the postcentral gyrus. Functional scans were acquired using a gradient-echo EPI sequence at $1.5 \times 1.5 \times 1.5 \mathrm{~mm}^{3}$ resolution $(\mathrm{TR}=2000 \mathrm{~ms}$, $\mathrm{TE}=36 \mathrm{~ms}$, flip angle $=70^{\circ}$, acquisition matrix $=128 \times 128$, field of view $=192 \times 192 \mathrm{~mm}^{2}$, partial Fourier factor $=6 / 8$ ). Identical intrasubject slice orientations between functional runs were ensured by applying the AutoAlign Scout program (provided by the manufacturer) prior to each run. Subjects underwent two MRI sessions, where the first session comprised anatomical and functional localizer scans and the second session focused on the four functional runs central to the question explored.

\section{Tactile stimulation and functional paradigms}

Tactile stimuli were delivered by a piezo-electric stimulation device (Piezostimulator, QuaeroSys, St. Johann, Germany). The system consists of a control unit plus five freely movable stimulation modules. Each of these modules contains an eight-dot Braille display $(2 \times 4$ matrix $)$ at the end of the top face, in which neighboring pins are divided by $2.5 \mathrm{~mm}$ covering an area of $2.5 \times 7.5 \mathrm{~mm}^{2}$ (Fig. 1A). In each cycle two randomly chosen pins were raised by $1.5 \mathrm{~mm}$. The stimulation frequency was set to $32 \mathrm{~Hz}$ (stimulation duration $=10.4 \mathrm{~ms}$, inter-stimulus inter$\mathrm{val}=20.8 \mathrm{~ms}$ ), eliciting mainly a sense of flutter which is transmitted by Meissner's corpuscles (Mountcastle et al., 1972; Talbot et al., 1968; McGlone and Reilly, 2010).

During functional imaging, subjects were keeping the hand relaxed and pronated. Each Braille display was positioned centrally below the respective part of the finger with the pin matrix oriented along the

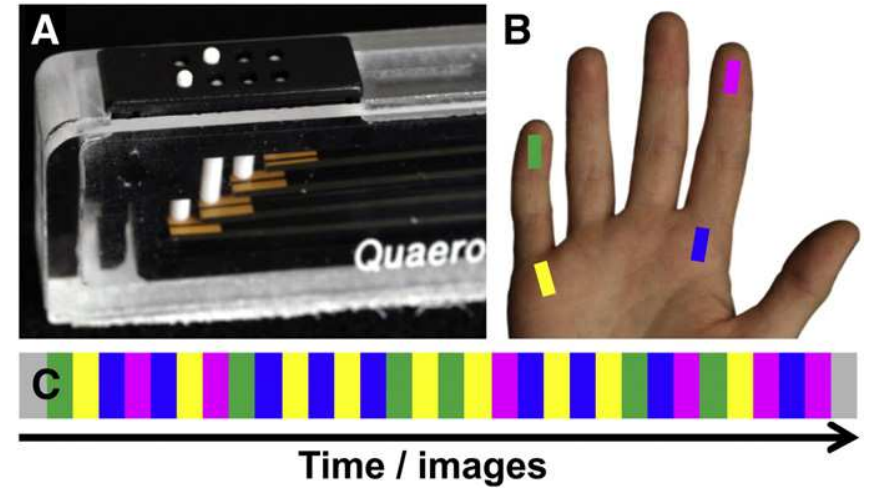

Fig. 1. Stimulation and paradigm. (A) Piezo-electric device used for vibrotactile stimulation of individual phalanges. (B) Stimulated hand areas for mapping: d2 p1 = magenta, d2 p4 = blue, d5 p1 = green, and d5 p4 = yellow. (C) Pseudo-randomized stimulation design (color code as above, baseline = gray).

finger axis and with the bulk of the module pointing distally for the fingertips and proximally for the bases. Optimal positioning was reassured before the experiment by test stimulation and individual adaptation of the height of the stimulation modules, especially for the base of $\mathrm{d} 2$ which otherwise might not touch the stimulator due to the relaxed posture of the hand. In order to draw and maintain attention of the subjects to the stimulation, short randomly distributed interrupts (length $156 \mathrm{~ms}$, occurring every 0.5 to $3 \mathrm{~s}$ ) were included in the stimulation intervals, which had to be counted and reported at the end of each run.

For each subject the first functional experiment served as a functional localizer for the representations of the five fingertips of the right hand. They were mapped by repetitive sequential stimulation of all fingertips from the thumb to the little finger each for a period of $12 \mathrm{~s}$ (6 images). Whenever four fingertips had been stimulated, a rest period of $12 \mathrm{~s}$ ( 6 images) was inserted to separate all neighboring finger stimulations from each other several times within the run.

The four functional runs that were central to the question of intradigit somatotopy were recorded in a second session. These runs were identical except for the sequence of the stimulation sites, which was pseudo-randomized. In each run the right-hand fingertips ( 1 1) and bases (p4) of the index finger (d2) and little finger (d5) were stimulated (Fig. 1B). Here, the term base refers to the volar skin position over the caput of the second and fifth metacarpal bone for $\mathrm{d} 2$ and $\mathrm{d} 5$, respectively.

Each run consisted of 6 baseline images $(12 \mathrm{~s})$ followed by a stimulation block of 180 images ( 6 min) plus a final rest period of 10 images (20 s). The stimulation block consisted of 30 stimulation trials of 6 images each (Fig. 1C). In each trial one of the four areas ( $\mathrm{p} 1$ and $\mathrm{p} 4$ for d2 and d5) was stimulated. Two trials for the same stimulation area were always separated by at least one other trial. As the fingertips were assumed to be more sensitive than the bases (Johansson and Vallbo, 1979), the stimulation block comprised only 6 trials for each fingertip but 9 trials for each base. The stimulation block of each of the four runs was obtained by pseudo-randomization on the above conditions.

\section{Preprocessing and coregistration}

Data analysis was carried out using BrainVoyager QX 2.1 (Brain Innovation, Maastricht, The Netherlands) (Goebel et al., 2006). The anatomical 3D dataset was transferred to ACPC space and a cortical mesh representing the white-to-gray matter border was reconstructed (Fig. 2A). For visualization purposes the mesh was inflated (Fig. 2B). No Talairach transformation was applied. All functional runs were motioncorrected in k-space (online software of the manufacturer) and 3Dmotion corrected and registered to the functional localizer using 

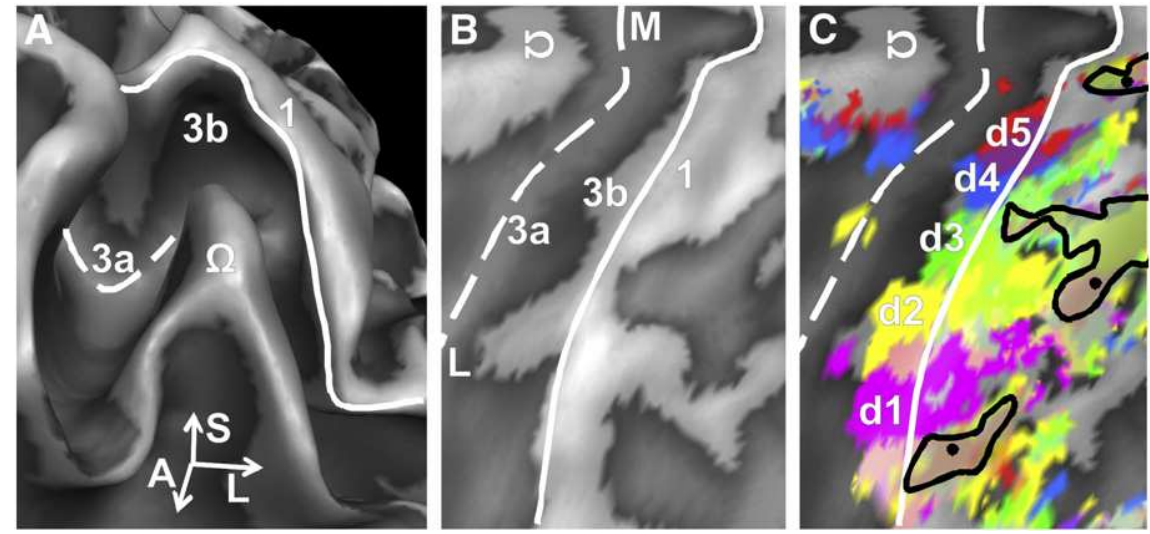

Fig. 2. Fingertip activations and vessel contributions. (A) Frontal superior view of the left-hemispheric primary somatosensory area ( $\Omega=$ motor hand knob) reconstructed at the white/ gray matter boundary (subject \#3). Convex and concave areas are shown in dark and light gray, respectively. BA = Brodmann areas $3 \mathrm{a}$, 3b, and 1 . The approximate fundus of the central sulcus is plotted as dashed line, the approximate transition between the anterior wall and the crown of the postcentral gyrus as plain line. (B) Inflated brain reconstruction of the area shown in (A) clockwise rotated by $90^{\circ}(\mathrm{M}=$ medial, $\mathrm{L}=$ lateral). (C) Fingertip activations for $\mathrm{d} 1=$ magenta, $\mathrm{d} 2=$ yellow, $\mathrm{d} 3=$ green, $\mathrm{d} 4=\mathrm{blue}$, and $\mathrm{d} 5=$ red yield mediolateral finger somatotopy in BA 3b. Vessel locations are drawn as black dots and respective "activations" are surrounded by black lines.

trilinear/sinc interpolation (BrainVoyager). Functional runs were highpass filtered and for each subject the functional localizer was coregistered to the anatomical image. The resulting coregistration matrices were fine-tuned by manual adjustments and applied to all functional runs of the subject for projecting them onto the T1-weighted 3D data in ACPC space at $1 \mathrm{~mm}$ isotropic resolution using trilinear interpolation.

In the present study the analysis was restricted to BA 3b. Similar to previous studies (Moore et al., 2000; Nelson and Chen, 2008) and in view of a missing in vivo method for determining the exact cytoarchitectonical transitions, BA $3 \mathrm{~b}$ was defined as the posterior wall of the central sulcus, although a high inter-subject variability exists for the exact locations of the borders both between BA 3a and $3 \mathrm{~b}$ and between BA 3b and 1 (Geyer et al., 1999).

The representations of the fingertips in BA $3 \mathrm{~b}$ were explored by calculating the contrast of the predictor (being convoluted with a twogamma function) for each fingertip against zero in a general linear model (GLM) analysis. The resulting activity maps were thresholded at a $\mathrm{t}$-value corresponding to a false discovery rate of $\mathrm{q}(\mathrm{FDR}) \leq 0.05$. Areas in which the $\mathrm{p} 1$ peak voxels of three or more digits were closely collocated (not further than $3 \mathrm{~mm}$ apart in any direction) were assumed to result from vessel "activations" (Fig. 2C) and respective contributions (at q $($ FDR $) \leq 0.05)$ were excluded from further analysis.

\section{Statistics}

For statistical analysis the design matrices (being convoluted with a two-gamma function) of the four $\mathrm{p} 1 / \mathrm{p} 4$ runs of a subject were concatenated and a fixed-effects GLM analysis was conducted. The contrasts of the predictors against zero (belonging to $\mathrm{p} 1$ and $\mathrm{p} 4$ for $\mathrm{d} 2$ and d5) were calculated, locating the activation elicited by the stimulation. The resulting maps were thresholded at a t-value corresponding to $\mathrm{q}(\mathrm{FDR}) \leq 0.05$. For illustrative purposes they were projected onto the inflated mesh of the respective subject. The centerof-mass coordinates (COM) and the number of activated voxels was determined for each of the calculated contrasts, taking into account all activated (possibly not connected) mesh areas close to the cortical representations identified for the fingertips. Data analysis was restricted to the region within $\mathrm{BA} 3 \mathrm{~b}$ that had not been excluded after the functional localizer.

Peak coordinates (PEAK, voxel with lowest p-value) for each of the four stimulation representations were identified in ACPC space, as these parameters have been suggested to best reflect the site of underlying neuronal activity (Arthurs and Boniface, 2003). Statistical tests were applied to assess the topography between the representation of fingertip and base for both fingers. To obtain normalized test quantities, the difference vectors between the PEAKs of fingertip and base contrasts were calculated using

$$
\text { Diff }=\left(\begin{array}{l}
x_{p 1}-x_{p 4} \\
y_{p 1}-y_{p 4} \\
z_{p 1}-z_{p 4}
\end{array}\right)
$$

for each finger. In order to test for somatotopy, the projections of the difference vector to the ACPC coordinate system were determined with $\mathrm{x}$ referring to the medial-lateral axis, $\mathrm{y}$ the anterior-posterior axis, and $z$ the inferior-superior axis: in humans, the rostrocaudal somatotopy expected from animal studies should manifest itself mainly in the $\mathrm{z}$ direction in $\mathrm{BA} 3 \mathrm{~b}$. For each axis the projection of the difference vector was tested for being different from zero using a twotailed $t$-test thresholded at $p \leq 0.025$ which results from a significance level of $95 \%$ corrected for the two finger comparisons in line with (Nelson and Chen, 2008). Further, the separation between respective p1 and p4 PEAKs was determined for each finger and subject by calculating the Euclidean length of the difference vector

$$
\sqrt{\left(x_{p 1}-x_{p 4}\right)^{2}+\left(y_{p 1}-y_{p 4}\right)^{2}+\left(z_{p 1}-z_{p 4}\right)^{2}} \text {. }
$$

For reasons described in Section 3, the Euclidean distances of d2 and $\mathrm{d} 5$ were compared in a two-tailed paired $t$-test thresholded at $p \leq 0.05$. COM coordinates were analyzed under the same statistical tests as described for PEAKs. In a final two-tailed paired $t$-test the number of activated voxels for $\mathrm{p} 4$ stimulation was compared between $\mathrm{d} 2$ and $\mathrm{d} 5$ at $p \leq 0.05$.

\section{Results}

Tactile stimulation of the right-hand fingertips and bases of $\mathrm{d} 2$ and d5 consistently led to activations within the expected hand area of SI in all subjects (Fig. 3). Activations in BA 3b were located mainly in the superior part of the posterior wall of the central sulcus. In many subjects more than one activation cluster was observed within BA $3 \mathrm{~b}$ for the same stimulation location (1.8 clusters per subject on average for each stimulation site).

The number of activated voxels decreased from $298 \pm 69$ for $\mathrm{p} 1$ to $183 \pm 44$ for $\mathrm{p} 4$ of the little finger as well as from $333 \pm 43$ for $\mathrm{p} 1$ to $111 \pm 34$ for $\mathrm{p} 4$ of the index finger. Activation foci for $\mathrm{p} 1$ were very 

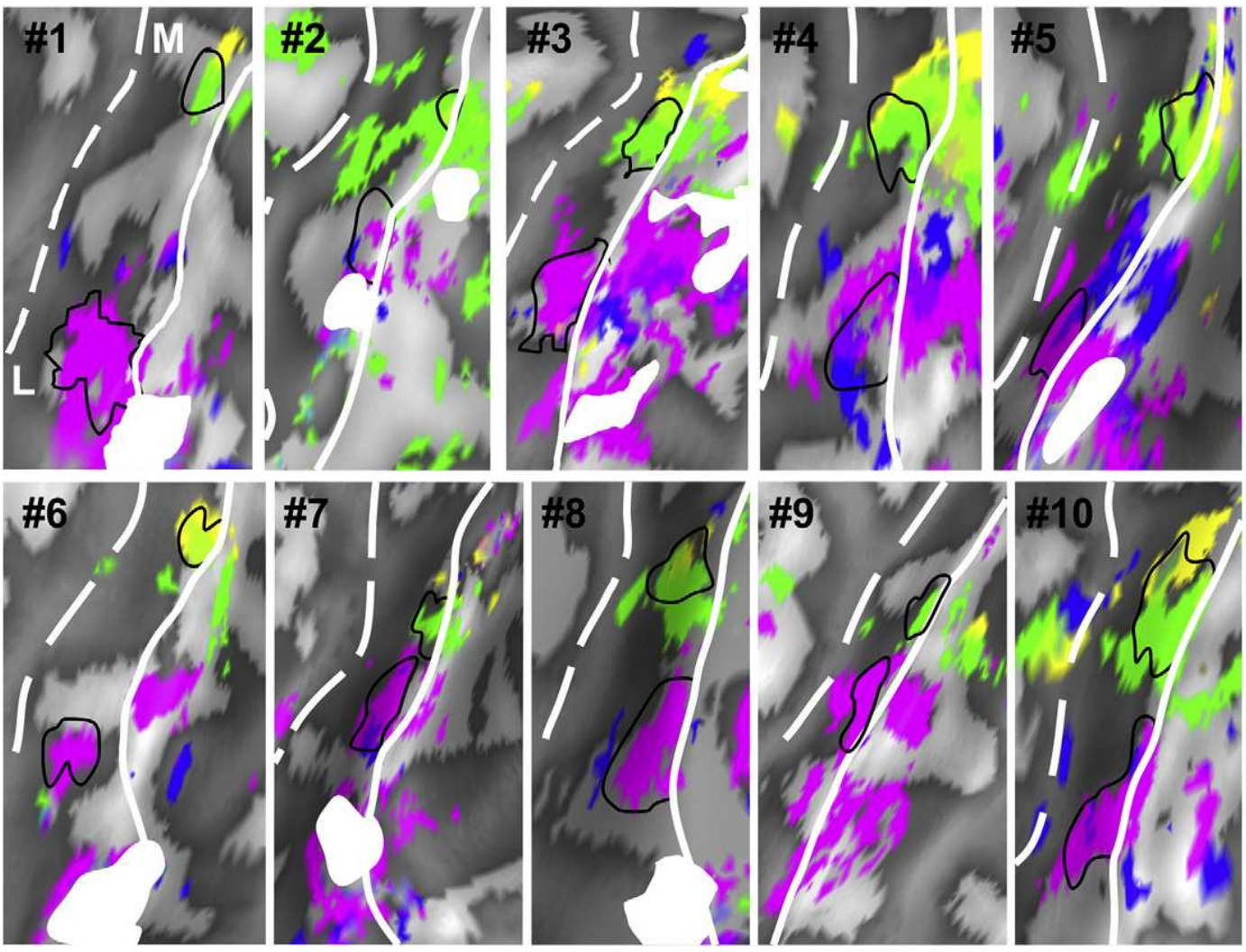

Fig. 3. Intra-digit topographic maps. Fingertip and base activations for the little and index finger for all subjects with $\mathrm{d} 5 \mathrm{p} 1=\mathrm{green}, \mathrm{d} 5 \mathrm{p} 4=\mathrm{yellow}, \mathrm{d} 2 \mathrm{p} 1=\mathrm{magenta}$, and $\mathrm{d} 2 \mathrm{p} 4=$ blue, overlap = respective intermediate color, excluded probable vessel "activations" = white (other parameters as in Fig. 2). BA 3b activations of the p1 representation elicited in the functional localizer are encircled in black (the more lateral and medial markers belong to d2 p1 and d5 p1, respectively). The little finger shows an intra-digit somatotopic arrangement.

similar to the activation of the respective fingertip observed with the functional localizer. Because the base representations also showed prominent activation in proximity of the respective fingertips, the $\mathrm{p} 1$ and $\mathrm{p} 4$ clusters for the same digit often overlapped (on average by $78 \pm 21$ voxels for $\mathrm{d} 5$ and $45 \pm 13$ voxels for $\mathrm{d} 2$ ).

The coordinates of the PEAK difference vectors ( $\mathrm{p} 1$ minus $\mathrm{p} 4$ ) are summarized in Table 1 . They were determined for each location and subject. The Euclidean distances between respective p1 and p4 PEAK representations yielded $5.0 \pm 0.7 \mathrm{~mm}$ (mean \pm SEM) for d5 and $6.7 \pm$ $0.5 \mathrm{~mm}$ for $\mathrm{d} 2$ indicating non-collocated representations. When testing whether $\mathrm{p} 4$ was significantly displaced relative to $\mathrm{p} 1$ along a certain direction, a somatotopic arrangement was observed for the little finger

Table 1

Difference coordinates and Euclidean distances between fingertip and base.

\begin{tabular}{|c|c|c|c|c|c|c|c|c|}
\hline \multirow[b]{3}{*}{ Subject } & \multicolumn{4}{|c|}{ Little finger (d5) } & \multicolumn{4}{|c|}{ Index finger (d2) } \\
\hline & \multicolumn{3}{|c|}{ Coordinates $/ \mathrm{mm}$} & \multirow{2}{*}{$\frac{\text { Euclidean }}{\text { Dist./mm }}$} & \multicolumn{3}{|c|}{ Coordinates/mm } & \multirow{2}{*}{$\frac{\text { Euclidean }}{\text { Dist./mm }}$} \\
\hline & $\mathrm{X}$ & $\mathrm{Y}$ & $\mathrm{Z}$ & & $\mathrm{X}$ & $\mathrm{Y}$ & $\mathrm{Z}$ & \\
\hline$\# 1$ & 1 & -5 & 1 & 5.2 & 3 & -4 & 3 & 5.8 \\
\hline$\# 2$ & -1 & -1 & 0 & 1.4 & 5 & -2 & -2 & 5.7 \\
\hline \#3 & 1 & -2 & 1 & 2.5 & -6 & 2 & 0 & 6.3 \\
\hline$\# 4$ & 1 & -1 & 1 & 1.7 & 4 & -6 & 0 & 7.2 \\
\hline \#5 & -1 & 3 & 5 & 5.9 & 0 & -2 & 5 & 5.4 \\
\hline \#6 & 2 & -5 & 2 & 5.7 & -3 & 2 & -2 & 4.1 \\
\hline \#7 & 3 & 3 & 3 & 5.2 & -1 & 6 & 3 & 6.8 \\
\hline$\# 8$ & 4 & -5 & -2 & 6.7 & 5 & -7 & -5 & 10.0 \\
\hline$\# 9$ & 5 & -1 & 6 & 5.2 & -4 & -2 & 7 & 8.3 \\
\hline$\# 10$ & 4 & -6 & 1 & 7.3 & 0 & 3 & 7 & 7.6 \\
\hline Mean & 1.9 & -2.0 & 1.8 & 5.0 & 0.3 & -1 & 1.6 & 6.7 \\
\hline \pm SEM & 0.7 & 1.0 & 0.7 & 0.7 & 1.2 & 1.3 & 1.3 & 0.5 \\
\hline $\mathrm{p}$ & $0.018^{*}$ & 0.085 & 0.038 & & 0.813 & 0.467 & 0.244 & \\
\hline
\end{tabular}

* Significant values $(\mathrm{p} \leq 0.025)$ are printed in bold. in mediolateral direction $(p=0.018)$, where $\mathrm{p} 4$ was represented $1.9 \pm$ $0.7 \mathrm{~mm}$ medial to $\mathrm{p} 1$, whereas significance was not reached in inferiorsuperior or anterior-posterior direction. Fig. 4 illustrates the intra-digit somatotopy of the little finger along the medial-lateral axis and a tendency for somatotopy in inferior-superior direction.

For some subjects (e.g., subject 9) this pattern cannot be observed on the inflated mesh (Fig. 3) despite its existence according to the PEAK analysis. This is due to several reasons. Firstly, limitations of the white/

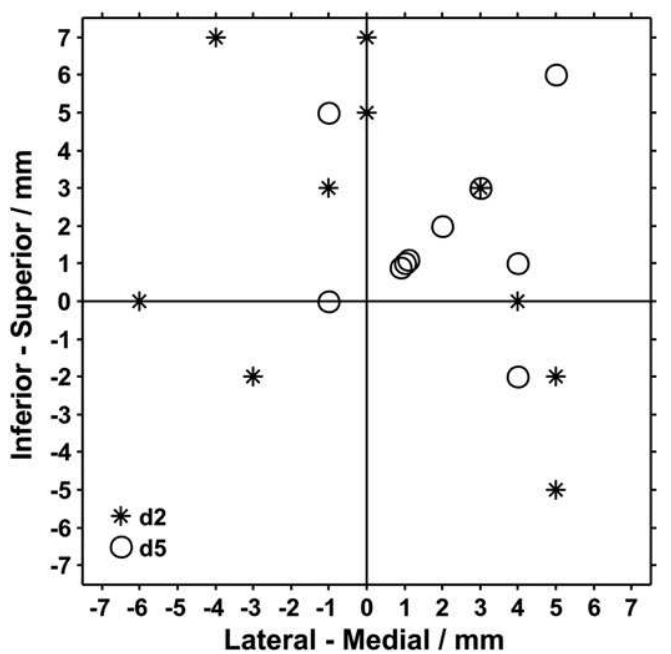

Fig. 4. Coronal separation coordinates. Coronal projection of difference coordinates for fingertip and base activations for $\mathrm{d} 2$ (asterisks) and $\mathrm{d} 5$ (open circles) of each subject. Positive abscissa and ordinate coordinates stand for $\mathrm{p} 4$ being represented medial and superior to $\mathrm{p} 1$, respectively. For $\mathrm{d} 5$, three subjects presented with coordinates $\mathrm{X}, \mathrm{Z}=1,1$ as indicated by overlapping circles. 
gray matter segmentation process and hence the mesh reconstruction impair the visibility of activations detected in the 3D volume map on the inflated mesh. In addition, during mesh inflation the 3D coordinate system is reduced to two dimensions mainly by combining anteriorposterior and inferior-superior axes. Hence, it becomes difficult to decide whether a structure lies posterior or superior to another. As a consequence, these maps were mainly used for illustrative purposes, whereas peak voxels were directly determined from 3D volume maps.

For the index finger no significant difference in the positions of $\mathrm{p} 1$ and $\mathrm{p} 4$ activations could be detected along any of the three orientations. However, the two-tailed paired $t$-test between the Euclidean distances ( $\mathrm{p} 1$ and $\mathrm{p} 4$ PEAK) for $\mathrm{d} 2$ and $\mathrm{d} 5$ showed that the distances were significantly larger for $\mathrm{d} 2$ than for $\mathrm{d} 5(p=0.039)$. Because $\mathrm{p} 1$ and $\mathrm{p} 4$ of d5 were already demonstrated to be non-collocated, this holds true for d2 as well.

In a second approach using COM instead of PEAK coordinates, $\mathrm{p} 1$ and $\mathrm{p} 4$ representations turned out to be apart by $4.1 \pm 0.5 \mathrm{~mm}$ for $\mathrm{d} 5$ and $4.5 \pm 0.7 \mathrm{~mm}$ for $\mathrm{d} 2$. Intra-digit somatotopy for the little finger reached significance not only in mediolateral $(p=0.005)$, but also in inferior-superior direction $(p=0.006)$ indicating a $\mathrm{p} 4$ location both medial and superior to p1. Again, no somatotopy was observed for the index finger. For the COMs, the Euclidean distances for $\mathrm{d} 2$ were not significantly different from the distances for $\mathrm{d} 5(p=0.722)$.

The $t$-test comparing the number of activated voxels for $\mathrm{p} 4$ stimulation between $\mathrm{d} 2$ and $\mathrm{d} 5$ missed significance $(p=0.065)$, but pointed into a direction of larger base activations for the little finger compared to the index finger.

\section{Discussion}

The present study explored intra-digit somatotopy using tactile stimulation and high-resolution fMRI. The study specifically focused on four areas of stimulation (fingertip and base of both index and little finger) not only to maximize the number of trials per stimulation area, but also to stimulate sites along the finger as distant from each other as possible. Furthermore, the analysis was restricted to BA $3 \mathrm{~b}$, firstly because cutaneous receptors mainly project their input via the thalamus to BA $3 \mathrm{~b}$ and less to BA 1, which receives its neuronal input from BA 3b (Powell and Mountcastle, 1968), and secondly because even in electrophysiological recordings in awake monkeys, which are expected to be more sensitive and precise than fMRI recordings in humans, no intra-digit pattern had been observed in BA 1 (Iwamura et al., 1983b).

The main results of the present study can be summarized as follows: (1) A consistent intra-digit somatotopic arrangement of the BA 3b representations of the fingertip (p1) and base (p4) was found across subjects for the little but not the index finger, and (2) in individual subjects, the PEAK representations of the fingertip and base in BA $3 \mathrm{~b}$ were clearly separated for both the little and the index finger.

In the little finger PEAK p4 was located significantly medial to p1. A similar cortical organization has been reported for macaques (Iwamura et al., 1983a), where the ulnar palmar skin (including the base of d5 and the adjacent half of $\mathrm{d} 4$ ) was found to be represented medially to the region for $\mathrm{d} 4$ and $\mathrm{d} 5$. In fact, extending the PEAK results, COM values reached significance also for the inferior-superior displacement of d5, suggesting that p4 may also be represented superior to $\mathrm{p} 1$ in humans. This inferior-superior organization resembles an analogous feature found in owl monkeys (Merzenich et al., 1978), where the base representation was found caudally (and not medially) to the respective fingertip. Thus, the current observations for $\mathrm{d} 5 \mathrm{seem}$ to be a combination of the arrangements found in the two monkey species.

On the contrary, the present data revealed no consistent topology of fingertip and base activations for the index finger across subjects. This observation is in contrast to monkey studies which claim that $\mathrm{p} 4$ of the index finger should be represented lateral to $\mathrm{p} 1$ in anesthetized macaques (Nelson et al., 1980) or caudal to $\mathrm{p} 1$ in awake macaques or anesthetized owl monkeys (Iwamura et al., 1983a; Merzenich et al., 1978).

This unexpected variance in the position of the index finger activations across subjects cannot solely be attributed to methodological reasons. The high isotropic resolution of $1.5 \mathrm{~mm}$ minimized partial volume effects, so that clear and significant BOLD activations for all four stimulation sites were observed for all subjects. In fact, these sites were proven to be reproducible in additional measuring sessions in three out of the 10 tested subjects (unpublished data, mean Euclidean difference between PEAK activations elicited by same stimulation point and subject: $1.8 \pm 0.6 \mathrm{~mm}$ ). The $1.5 \mathrm{~mm}$ resolution was further adequate to resolve the distance between $\mathrm{p} 1$ and $\mathrm{p} 4$, because the respective Euclidean distances for the little and index finger were $5.0 \mathrm{~mm}$ and $6.7 \mathrm{~mm}$, respectively. The finding of lower numbers of activated voxels for $\mathrm{p} 4$ in comparison to $\mathrm{p} 1$ at both fingers also demonstrates the reliability of the data. This difference reflects smaller cortical representations for the bases, which are caused by the lower number of tactile receptors at the bases compared to the fingertips (Johansson and Vallbo, 1979).

A possible caveat in identifying intra-digit somatotopy for $\mathrm{d} 2$ might be the complex spatial structure of the postcentral gyrus, which shows a sharp bend at the location of the $\mathrm{d} 2$ representation in most subjects. Therefore, a somatotopic arrangement might have been missed when quantifying somatotopy via the ACPC-coordinate system. However, if this were the case, one should still be able to visually identify somatotopy on the inflated cortical surface, which we did not observe.

Possible differences in stimulation intensity also have to be taken into account. Seven out of 10 subjects reported to feel the 4 stimulation of the index finger much weaker than the other three stimulation points. Although we cannot entirely exclude a potential problem in the positioning of the respective piezo stimulator, especially as the element was completely hidden by the palm and thumb, we optimized the positioning procedure by touching the dorsal part of the hand "above" p4 and asking the subjects to report whether the stimulation occurred below this site of touch. More likely, the difference in perceived stimulus intensity between the two fingerbases reflects an essential difference in importance of these two locations in our everyday use of the hand. This is because the base of the little finger delineates an outer border of the hand which is involved in perception of most objects, whereas the base of the index finger only perceives sensory information when manipulating convex objects and does not get into touch with flat objects in the natural posture of the hand. The difference in perception is mirrored in a trend in the number of activated voxels being higher for $\mathrm{p} 4$ of the little finger than for the index finger, as well as a smaller difference in the number of activated voxels between $\mathrm{p} 1$ and $\mathrm{p} 4$ at $\mathrm{d} 5$ than at $\mathrm{d} 2$. Both the increased perceived stimulus intensity and the higher number of activated voxels would point to a higher number of cutaneous receptors at $\mathrm{p} 4$ of the little finger compared to $\mathrm{p} 4$ of the index finger, but to our knowledge there are no data available. Finally, it is conceivable that the behavioral (sensory) importance of the base of the little finger (and possibly of the thumb at the other side of the hand) is not only reflected in an increased number of tactile receptors in the periphery and a larger cortical representation, but also in a more well-defined somatosensory map compared to that of other fingers.

Consistency or inconsistency of somatotopic maps across individual subjects has so far not been considered as a relevant feature. Most studies of somatosensory areas in animals (Merzenich et al., 1978; Kaas et al., 1979; Nelson et al., 1980; Iwamura et al., 1983a, 1983b) and in humans (Penfield and Rasmussen, 1950; Maldjian et al., 1999; Kurth et al., 2000; Nelson and Chen, 2008) focused on the description of more general somatotopic features, which are expected to be consistent across individuals of the same species. Individual deviations such as alterations of the usual sequence of finger representations have only rarely been described in humans (Kurth et al., 1998; Schweizer et al., 2008). In contrast, Merzenich et al. (1987) described a considerable individual variability for different aspects of the topography of the hand area both in owl and squirrel monkeys as revealed by electrophysiological mapping 
at high spatial resolution. This idiosyncratic variability existed despite the preservation of somatotopic features such as the sequence of finger representations. The present high-resolution fMRI data allow for a similar description of topographic inconsistencies in parts of the hand area across human subjects.

At this stage the definite reasons and mechanisms for the absence of an intra-digit somatotopy of the human index finger remain unclear. Merzenich et al. (1987) proposed that the various individual differences in the detailed cortical map structure cannot solely be accounted for by ontogenetically defined maps, but also depend on the individual life-long use of the hand, forming an individual topography in the somatosensory map. In order to follow up along these lines, it seems most important to investigate a possible intradigit somatotopy for other fingers including the thumb, which forms the edge of the hand opposite to d5. More detailed studies of the individual maps of the complete digit representations of all fingers will not only give insight into the variability within the human somatosensory hand area, but also account for the possible influence of general versus specific hand use on the formation of topographic somatosensory maps. In addition, it might be insightful to study both the dominant and non-dominant hand as they might be subject to a different balance of genetic and behavioral influences.

\section{Funding}

This work was supported by the International Max Planck Research School MSc/PhD/MD-PhD Neuroscience Program (to M.A.S.) of the Göttingen Graduate School of Neurosciences and Molecular Biosciences (GGNB) in Göttingen, Germany, and the graduate school Neurosenses (to M.A.S.) in Göttingen and Oldenburg, Germany.

\section{References}

Arthurs, O.J., Boniface, S.J., 2003. What aspect of the fMRI BOLD signal best reflects the underlying electrophysiology in human somatosensory cortex? Clin. Neurophysiol. $114,1203-1209$.

Blankenburg, F., Ruben, J., Meyer, R., Schwiemann, J., Villringer, A., 2003. Evidence for a rostral-to-caudal somatotopic organization in human primary somatosensory cortex with mirror-reversal in areas 3b and 1. Cereb. Cortex 13, 987-993.

Brodmann, K., 1909. Vergleichende Lokalisationslehre der Grosshirnrinde. Barth, Leipzig(DE).

Geyer, S., Schleicher, A., Zilles, K., 1999. Areas 3a, 3b, and 1 of human primary somatosensory cortex: 1 . Microstructural organization and interindividual variability. Neuroimage 10, 63-83.

Goebel, R., Esposito, F., Formisano, E., 2006. Analysis of functional image analysis contest (FIAC) data with brainvoyager QX: from single-subject to cortically aligned group general linear model analysis and self-organizing group independent component analysis. Hum. Brain Mapp. 27, 392-401.

Hashimoto, I., Mashiko, T., Kimura, T., Imada, T., 1999a. Are there discrete distalproximal representations of the index finger and palm in the human somatosensory cortex? A neuromagnetic study. Clin. Neurophys. 110, 430-437.

Hashimoto, I., Saito, Y., Iguchi, Y., Kimura, T., Fukushima, T., Terasaki, O., Sakuma, K., 1999b. Distal-proximal somatotopy in the human hand somatosensory cortex: a reappraisal. Exp. Brain Res. 129, 467-472.

Hlushchuk, Y., Forss, N., Hari, R., 2004. Distal-to-proximal representation of volar index finger in human area 3b. Neuroimage 21, 696-700.

Iwamura, Y., Tanaka, M., Sakamoto, M., Hikosaka, O., 1983a. Functional subdivisions representing different finger regions in area 3 of the first somatosensory cortex of the conscious monkey. Exp. Brain Res. 51, 315-326.
Iwamura, Y., Tanaka, M., Sakamoto, M., Hikosaka, O., 1983b. Converging patterns of finger representation and complex response properties of neurons in area 1 of the first somatosensory cortex of the conscious monkey. Exp. Brain Res. 51, 327-337.

Johansson, R.S. Vallbo, A.B. 1979. Tactile sensibility in the human hand: relative and absolute densities of four types of mechanoreceptive units in glabrous skin. J. Physiol. 286, 283-300.

Kaas, J.H., Nelson, R.J., Sur, M., Lin, C.S., Merzenich, M.M., 1979. Multiple representations of the body within the primary somatosensory cortex of primates. Science 204, 521-523.

Kurth, R., Villringer, K., Mackert, B.M., Schwiemann, J., Braun, J., Curio, G., Villringer, A., Wolf, K.J., 1998. fMRI assessment of somatotopy in human Brodmann area 3b by electrical finger stimulation. Neuroreport 9, 207-212.

Kurth, R., Villringer, K., Curio, G., Wolf, K.J., Krause, T., Repenthin, J., Schwiemann, J., Deuchert, M., Villringer, A., 2000. fMRI shows multiple somatotopic digit representations in human primary somatosensory cortex. Neuroreport 11, $1487-1491$

Maldjian, J.A., Gottschalk, A., Patel, R.S., Detre, J.A., Alsop, D.C., 1999. The sensory somatotopic map of the human hand demonstrated at 4 Tesla. Neuroimage 10 , 55-62.

McGlone, F., Reilly, D., 2010. The cutaneous sensory system. Neurosci. Biobehav. Rev. 34, 148-159.

Merzenich, M.M., Kaas, J.H., Sur, M., Lin, C.-S., 1978. Double representation of the body surface within cytoarchitectonic area 3b and 1 in SI in the owl monkey (aotus trivirgatus). J. Comp. Neurol. 181, 41-73.

Merzenich, M.M., Nelson, R.J., Kaas, J.H., Stryker, M.P., Jenkins, W.M., Zook, J.M., Cynader, M.S., Schoppmann, A., 1987. Variability in hand surface representations in areas 3b and 1 in adult owl and squirrel monkeys. J. Comp. Neurol. 258, 281-296.

Moore, C.I., Stern, C.E., Corkin, S., Fischl, B., Gray, A.C., Rosen, B.R., Dale, A.M., 2000. Segregation of somatosensory activation in the human rolandic cortex using fMRI. J. Neurophysiol. 84, 558-569.

Mountcastle, V.B., LaMotte, R.H., Carli, G., 1972. Detection thresholds for stimuli in humans and monkeys: comparison with threshold events in mechanoreceptive afferent nerve fibers innervating the monkey hand. J. Neurophysiol. 35, 122-136.

Nelson, A.J., Chen, R., 2008. Digit somatotopy within cortical areas of the postcentral gyrus in humans. Cereb. Cortex 18, 2341-2351.

Nelson, R.J., Sur, M., Felleman, D.J., Kaas, J.H., 1980. Representations of the body surface in postcentral parietal cortex of Macaca fascicularis. J. Comp. Neurol. 192, 611-643.

Oldfield, R.C., 1971. The assessment and analysis of handedness: the Edinburgh inventory. Neuropsychologia 9, 97-113.

Overduin, S.A., Servos, P., 2004. Distributed digit somatotopy in primary somatosensory cortex. Neuroimage 23, 462-472.

Paul, R.L., Merzenich, M.M., Goodman, H., 1972. Representation of slowly and rapidly adapting cutaneous mechanoreceptors of the hand in Brodmann's areas 3 and 1 of Macaca mulatta. Brain Res. 36, 229-249.

Penfield, W., Boldrey, E., 1937. Somatic motor and sensory representation in the cerebral cortex of man as studied by electrical stimulation. Brain 60, 389-443.

Penfield, W., Rasmussen, T., 1950. The Cerebral Cortex of Man: A Clinical Study of Localization of Function. Macmillan, New York

Powell, T.P.S., Mountcastle, V.B., 1968. Some aspects of the functional organization of the cortex of the postcentral gyrus of the monkey: a correlation of findings obtained in a single unit analysis with cytoarchitecture. Bull. Johns Hopkins Hosp. 105, 301-334.

Sanchez-Panchuelo, R.M., Francis, S., Bowtell, R., Schluppeck, D., 2010. Mapping human somatosensory cortex in individual subjects with 7T functional MRI. J. Neurophysiol. 103, 2544-2556.

Schweizer, R., Voit, D., Frahm, J., 2008. Finger representations in human primary somatosensory cortex as revealed by high-resolution functional MRI of tactile stimulation. Neuroimage 42, 28-35.

Talbot, W.H., Darian-Smith, I., Kornhuber, H.H., Mountcastle, V.B., 1968. The sense of flutter-vibration: comparison of the human capacity with response patterns of mechanoreceptive afferents from the monkey hand. J. Neurophysiol. 31, 301-334.

Tanosaki, M., Hashimoto, I., 2004. Serial N20m dipoles in somatosensory evoked magnetic fields move along the distal-proximal representation of the digit area $3 \mathrm{~b}$ of the human cortex. Neurosci. Lett. 359, 175-179.

Vogt, C., Vogt, O., 1919. Allgemeinere Ergebnisse unserer Hirnforschung. J. Psychol. Neurol. 25, 279-462.

Yousry, T.A., Schmid, D.U., Alkadhi, H., Schmidt, D., Peraud, A., Buettner, A., Winkler, P. 1997. Localization of the motor hand area to a knob on the precentral gyrus. A new landmark. Brain 120, 141-157. 



\subsection{Functional MRI reveals individual variations in the complete map of human digit phalanges in the primary somatosensory}

\section{cortex}

In this chapter, intra-digit somatotopy was explored, for a first time mapping all phalanges of all digits of the dominant hand, in a single imaging session. Across digits, the well-known succession of representations from little finger to thumb could not only be confirmed for the fingertips but for the first time also be shown for the middle and proximal phalanges. Within a digit, ordered BA $3 \mathrm{~b}$ representations from tip over medial to proximal phalanx along an axis consistent across subjects could only be observed in the little finger and, in a trend, for the ring finger, whereas maps in index finger, middle finger, and thumb varied between subjects. These findings corroborate and extend the results of Chapter 2.1 and again point to an inverse relation between individuality of digit use and across-subjects consistency of cortical intra-digit maps. 
Functional MRI reveals individual variations in the complete map of human digit phalanges in the primary somatosensory cortex

Complete digit map of human BA 3b

Meike A. Schweisfurth ${ }^{1,2}$, Jens Frahm ${ }^{1}$, Renate Schweizer ${ }^{1}$

${ }^{1}$ Biomedizinische NMR Forschungs $\mathrm{GmbH}$ am Max-Planck-Institut für biophysikalische Chemie, 37070 Göttingen, Germany, ${ }^{2}$ Cognitive Neuroscience Laboratory, German Primate Center, 37077 Göttingen, Germany

Correspondence should be addressed to: Meike Schweisfurth, Biomedizinische NMR Forschungs GmbH, 37070 Göttingen, Germany; Email: mschwei1@gwdg.de 
2.2 Functional MRI reveals individual variations in the complete map of human digit phalanges in the primary somatosensory cortex

\section{Abstract}

High-resolution functional magnetic resonance imaging (fMRI) was used to map the entire digit area of the right, dominant hand in Brodmann area $3 \mathrm{~b}$ of the human somatosensory cortex. The paradigm employed tactile stimulation of the phalanges and bases of all five digits in a single fMRI session and resulted in ACPC coordinates of respective activation peak vertices on individual cortical surface reconstructions.

A principal component analysis identified individual directions aligning activation peaks of either homologous digit phalanges or different phalanges within single digits. An additional analysis tested whether phalanges were ordered along the resulting direction vector, i.e. from the little finger (D5) to the thumb (D1) across digits or from the first ( $p 1)$ to the third phalanx (p3) within digits.

The across-digit analysis showed the expected ordered succession of the first phalanges of the five digits along a common direction. The same ordered succession could be demonstrated for the second and third phalanges, confirming the somatotopy of digit representations along the hand area. For the intra-digit analysis, results diverged between different digits, revealing intra-digit direction consistency across the phalanges only for D5 and D4 and ordering of the phalanges only for D5. These findings may be explained by the more uniform use of the "supporting" digits D4 and D5, contrasted by the individually varying use of the digits D1 to D3 of the dominant hand, the latter resulting in individualized intra-digit cortical maps.

Keywords: Across-digit map, BA 3b, fMRI, human, intra-digit map 


\section{Introduction}

Primary sensory cortices are characterized by topographic maps which represent the peripheral receptor sheet in a highly ordered fashion (Kaas, 1997). In auditory cortex, frequency is encoded along a single tonotopic axis, whereas the retinotopic map in primary visual cortex incorporates two perpendicular visual-field axes to encode eccentricity and polar angle. The somatotopy of the human primary somatosensory cortex (SI), first described by Penfield's somatosensory homunculus (Penfield and Boldrey, 1937; Penfield and Rasmussen, 1950), comprises the tactile body-surface representation along the postcentral gyrus, representing the digits D5 (little finger) to D1 (thumb) in medial-to-lateral succession. Electrophysiological mapping of the hand area in anesthetized primates implies a second axis perpendicular to the first, representing the phalanges within a digit (Merzenich et al., 1978; Kaas et al., 1979; Nelson et al., 1980), with the distal phalanx ( $p 1$ ) being represented towards the Brodmann area (BA) $3 a / 3 b$ border and the proximal parts of the digit towards the BA $3 \mathrm{~b} / 1$ border. In awake macaques, this clear arrangement could not entirely be reproduced. Neurons with receptive fields for the proximal phalanges were still found close to the BA $3 b / 1$ border, whereas representations of distal phalanges were not limited towards $B A$ 3a but present across the entire respective digit's BA 3b strip (Iwamura et al., 1983).

In humans, functional magnetic resonance imaging (fMRI) confirmed the medial-to-lateral D5-to-D1 succession of the distal phalanx in BA 3b (e.g. Nelson et al., 2008; Schweizer et al., 2008; Sanchez-Panchuelo et al., 2010). However, attempts to obtain intra-digit maps of single digits delivered inconsistent results. Stimulating p1 to p4 (digit base) of D3, Blankenburg and colleagues (2003) found in a group analysis a BA $3 b$ succession of p1-to-p4 activations towards the BA 3b/1 border, similar to the intra-digit somatotopy obtained in owl monkeys (Merzenich et al., 1978). Analyzing individual maps we found that p4 of D5 was consistently located medial to 11 in BA $3 b$, whereas no consistent across-subjects arrangement was observed for D2 (Schweisfurth et al., 2011). Sanchez-Panchuelo et al. (2012) applied the traveling-wave approach (Sereno et al., 1994) to the phalanges and base of D2 and showed activation patterns in which they identify a mirror-reversal pattern 
2.2 Functional MRI reveals individual variations in the complete map of human digit phalanges in the primary somatosensory cortex

for p1-to-p3 and p3-to-p1 across SI in 4 out of 6 subjects, suggesting an ordering similar as in anesthetized macaques (Nelson et al., 1980).

Contrasting these studies of only single digits, we aimed to obtain complete BA $3 b$ intra-digit maps for all five digits of individual subjects in one fMRI session. The approach was chosen to investigate the general outline of BA $3 \mathrm{~b}$ maps as well as to allow for the description of individual variations. To compare individual BOLDactivation maps across subjects, principle component analyses were applied to the across-digit or intra-digit locations of peak activation. The resulting vectors describe the direction of the succession of homologous phalanges across digits or of different phalanges within a digit. After assessing whether successive activations were ordered along these vectors, the direction consistency and ordering of the intra-digit and across-digit maps were statistically explored across subjects. 


\section{Materials and Methods}

\section{MRI}

Approval for the present study was obtained from the local ethics committee. After informed written consent before each session 18 healthy subjects (6 women, range 21 to 30 years, mean $27 \pm 2.5$ years) participated in two MRI sessions. All subjects were right-handed according to the Edinburgh Inventory (laterality index $0.9 \pm 0.1$, Oldfield, 1971). Magnetic resonance imaging (MRI) was performed at $3 \mathrm{~T}$ (TIM Trio, Siemens Healthcare, Erlangen, Germany) using a 32-channel head coil. The first session incorporated whole-brain structural MRI, partial-volume MR angiography, whole-brain single-image echo-planar imaging (EPI), and a partialvolume functional-localizer $\mathrm{fMRI}$ measurement for delineating the presumed digitrepresentation area along the central sulcus. The second session consisted of $5 \mathrm{fMRI}$ measurements, each mapping the phalanges within a single digit.

Structural MRI was based on sagittal T1-weighted 3D MPRAGE (magnetization-prepared rapid gradient-echo) acquisition (repetition time (TR) = $2530 \mathrm{~ms}$, echo time $(T E)=3.4 \mathrm{~ms}$, flip angle $=7^{\circ}$, acquisition matrix $=256 \times 256$, $160-192$ partitions, resolution $=1 \times 1 \times 1 \mathrm{~mm}^{3}$, total acquisition time $(\mathrm{TA})=$ 10:49 min). The image was used to identify the motor hand knob at the precentral gyrus (Yousry et al., 1997) and to perform surface reconstructions of the cortical white-matter / gray-matter boundary.

Functional images were recorded using a gradient-echo EPI sequence at $1.5 \times 1.5 \times 1.5 \mathrm{~mm}^{3}$ resolution $\left(\mathrm{TR}=2000 \mathrm{~ms}, \mathrm{TE}=36 \mathrm{~ms}\right.$, flip angle $=70^{\circ}$, acquisition matrix $=128 \times 128$, field of view $=192 \times 192 \mathrm{~mm}^{2}$, partial Fourier factor $=$ 6/8). Nineteen double-oblique transverse-to-sagittal and transverse-to-coronal sections were positioned perpendicular to the wall of the central sulcus, cutting the motor hand knob in mediolateral direction and covering its whole depth. The AutoAlign Scout feature (Siemens Healthcare, Erlangen, Germany) ensured an identical slice position for all consecutive fMRI runs for the individual subject.

A single gradient-echo EPI volume was performed with identical orientation and position as the functional measurements, but covering the entire brain with 81 slices $\left(1.5 \times 1.5 \times 1.5 \mathrm{~mm}^{3}\right.$ resolution, TR $=8600 \mathrm{~ms}$, TE $=36 \mathrm{~ms}$, flip angle $=$ $70^{\circ}$, acquisition matrix $=128 \times 128$, field of view $=192 \times 192 \mathrm{~mm}^{2}$, partial Fourier 
2.2 Functional MRI reveals individual variations in the complete map of human digit phalanges in the primary somatosensory cortex

factor $=6 / 8)$, to facilitate registration of the partial-volume images to the wholevolume structural images. MR angiography was based on a T1-weighted 3D FLASH sequence $\left(T R=22 \mathrm{~ms}, T R=4.43 \mathrm{~ms}\right.$, flip angle $=18^{\circ}$, resolution $=0.3 \times 0.3$ $\times 0.5 \mathrm{~mm}^{3}, 57$ sections from two overlapping slabs) with identical volume coverage and slice orientation as the functional images.

For three of the subjects, the fMRI session for the mapping of the phalanges was repeated to assess the retest-reliability of the resulting maps by calculating the Euclidean distance between the peak-vertex coordinates of the first and second session.

\section{Tactile stimulation}

Tactile stimulation was applied to the first, second, and third phalanx ( $p 1, p 2$, p3) and to the base (p4) of each digit. The term "base" refers to the volar pads described in primate studies (Merzenich et al., 1978) and denotes the palmar skin position over the caput of the metacarpal bone of the respective digit (Blankenburg et al., 2003). The four stimulation sites at D5 through D2 and the three sites at D1 were stimulated separately. Tactile stimulation was applied as described previously (Schweizer et al., 2008; Schweisfurth et al., 2011) by a piezo-electric unit (QuaeroSys, St. Johann, Germany) equipped with 5 independent stimulation modules, each with an 8-dot Braille display $(2 \times 4$ matrix, covering an area of 2.5 $\times 7.5 \mathrm{~mm}^{2}$ ) at its distal top face (Fig. 1A).

For the digit localizer measurement, the 5 stimulation modules were individually positioned under the distal phalanx (p1) of each digit, with the Braille display being oriented centrally below the phalanx, parallel to the digit axis, and with the bulk of the module pointing away from the digit. For intra-digit phalanx mapping, the 4 stimulation modules were positioned centrally below the phalanx, but orthogonally to the digit axis (Fig. 1B). For stimulation of the phalanges of D2 and D3 the bulk of the modules pointed to the ulnar, for D4 and D5 to the radial, and for D1 to the ulnar (digit base and p2) and distal (p1) side.

In general, subjects were told to keep their right hand relaxed and pronated. Before each fMRI measurement, it was confirmed that subjects clearly perceived the stimulation at each location. Stimulation frequency was set to $32 \mathrm{~Hz}$. In each cycle two out of the 8 pins were randomly chosen and elevated (square wave, stimulation 
duration $=10.4 \mathrm{~ms}$, inter-stimulus interval $=20.8 \mathrm{~ms}$ ) resulting in a fast varying stimulation pattern across the entire Braille display. The pins were set to maximum drive-out such that the tactile stimulation was clearly detectable and as salient as possible to be able to elicit an assessable BOLD response for each stimulation site in each subject. Each functional measurement was also associated with a task in which subjects had to covertly count short randomly distributed interrupts during tactile stimulation (duration $156 \mathrm{~ms}$, repetition every 0.5 to $3 \mathrm{~s}$ ) to assure attention on the stimulation. After completion of the measurement subjects reported the summed number of interrupts and received verbal feedback by the experimenter about their potential deviation from the correct number.

The localizer mapping employed sequential stimulation of the distal phalanges of the right hand from the thumb to the little finger with a rest period after each fourth digit stimulation (D1, D2, D3, D4, Rest, D5, D1, D2, D3, Rest, D4, D5, D1, D2...). The duration of stimulation and rest periods was $12 \mathrm{~s}$ (6 images). Each of the 5 digits
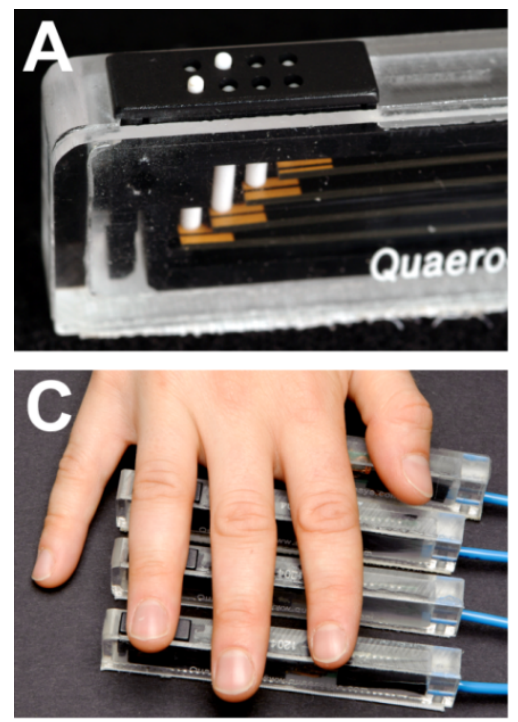

B

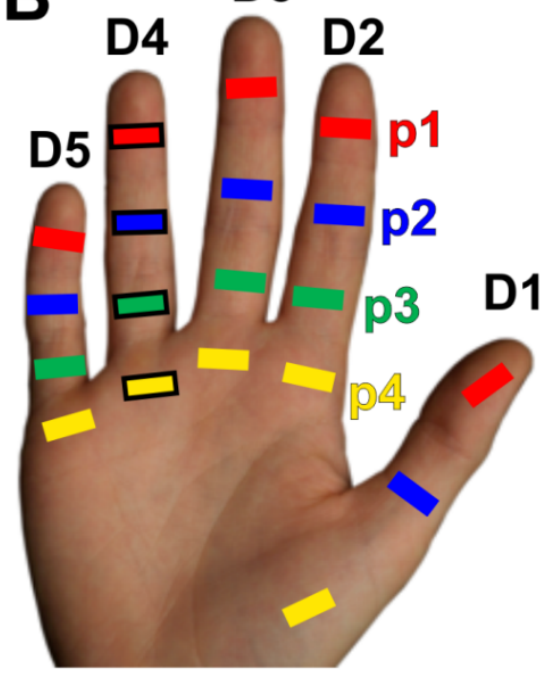

\section{D}

$0 \quad 20 \quad 40 \quad 60 \quad 80 \quad 100120140160180200220240260280$

\section{Time / images}

Figure 1. Tactile stimulation (A) Piezo-electric device used for vibrotactile stimulation of individual phalanges. (B) Stimulated digit areas for mapping $\mathrm{p} 1=$ red, $\mathrm{p} 2=$ blue, $\mathrm{p} 3=$ green, and $\mathrm{p} 4=$ yellow. One digit was stimulated per run, the example in (C) refers to the ring finger (black frames). (C) Positioning of the piezo-electric devices to the ring finger. (D) Pseudo-randomized stimulation design (color code as above, baseline $=$ grey). was stimulated during 8 periods $($ total $=96 \mathrm{~s}$, 48 images) which together with 10 rest periods $($ total $=120 \mathrm{~s}$, 60 images) leads to a total scan time of 10:20 min (310 images) including baseline $(20 \mathrm{~s}$, 10 images).

The second MRI session consisted of 5 functional runs, each exploring the topography of the phalanges within a single digit. In each run the first ( $p 1)$, the second (p2), and the third (p3, except for the thumb) 
2.2 Functional MRI reveals individual variations in the complete map of human digit phalanges in the primary somatosensory cortex

phalanx and the base (p4) of the respective digit were stimulated (Fig. 1C). Runs for neighboring digits were separated by at least one other run. Each functional measurement (Fig. 1D) consisted of 6 cycles of a stimulation block (average: $84 \mathrm{~s}$, 42 images) alternating with a rest block (average: $12 \mathrm{~s}, 6$ images). During each stimulation block each of the 3 phalanges and the base were stimulated for 8,12 , or $16 \mathrm{~s}$ (average: $12 \mathrm{~s}$ ). The distal phalanx (p1) was stimulated once and each of the other phalanges (p2, p3) and the base (p4) twice within the block to account for the lower sensitivity of p2, p3 and p4 (Johansson and Vallbo, 1979; Schweisfurth et al., 2011). The succession of the 4 stimulation sites within the stimulation block was pseudo-randomized such that the stimulation of one phalanx was always followed by the stimulation of another phalanx or a rest block. Total scan time for one functional measurement was 9:56 min or 298 images (7:32 min, 226 images for D1) including baseline (20 s, 10 images). The resulting total stimulation time for the first phalanx (p1) was $72 \mathrm{~s}$ (36 images) and $144 \mathrm{~s}$ (72 images) for p2, p3, and p4.

\section{Preprocessing and co-registration}

The first part of data analysis was performed with BrainVoyager QX 2.3 (Goebel et al., 2006; Brain Innovation, Maastricht, The Netherlands). Structural T1-weighted images were translated and rotated such that the axial slice orientation was parallel to the plane through the anterior and posterior commissures (AC-PC plane) and corrected for intensity inhomogeneities. The resulting image was used to reconstruct the cortical mesh at the white-matter gray-matter border by applying the BrainVoyager surface-reconstruction tool. Functional runs were motion-corrected in k-space (Siemens Healthcare, Erlangen, Germany) and image space (BrainVoyager) as well as temporally high-pass filtered as implemented as default in BrainVoyager. In the same step, each functional run of one subject was registered to the functional localizer of the first session (using trilinear/sinc interpolation) to ensure an accurate alignment between all functional measurements within a subject. The first functional measurement of each subject was automatically registered ( 6 degrees of freedom) to the individual whole-brain T1-weighted structural image; additional manual optimization ensured its best possible registration specifically in the region of the central sulcus. The resulting registration matrix was then applied to each functional run of the subject to achieve identical projections onto the T1-weighted 3D data in 
ACPC space (using trilinear interpolation). During registration the fMRI data were also interpolated from $1.5 \mathrm{~mm}$ isotropic resolution to $1 \mathrm{~mm}$ isotropic resolution.

Within FSL (FMRIB Software Library, Smith et al., 2004), the MR angiography volume was projected onto the T1-weighted image in ACPC space. After import of the structural images from BrainVoyager, both the T1-weighted image and the MR angiogram were brain-extracted (BET) and interpolated to $0.5 \mathrm{~mm}$ isotropic resolution. Several sequential registration steps were carried out using FLIRT (FMRIB's Linear Image Registration Tool, Jenkinson and Smith, 2001). The first volume of the functional localizer measurement was registered both to the MR angiogram (matrix M_1, 7 DOF) and to the whole-brain EPI acquisition (matrix M_2, 6 DOF). Then, the whole-brain EPI scan was registered to the structural T1-weighted image (matrix M_3, 7 DOF). Concatenating the inverse of M_1 with M_2 and M_3, the MR angiogram was projected onto the $0.5 \mathrm{~mm}$ isotropic T1-weighted image in BrainVoyager ACPC space.

The next step involved the exclusion of voxels around prominent blood vessels, a method routinely applied in human visual high-resolution fMRI studies (Cheng et al., 2001; Yacoub et al., 2001; Shmuel et al., 2007) to reduce the BOLD signal from these vessels, limiting the spatial specificity of the BOLD response (see Polimeni et al., 2010 for the visual system). For the somatosensory system this was based on a two-step approach. Using the functional localizer, fMRI activation clusters in which the peak voxels (of an activation cluster) of three or more digits were in very close proximity (not further than $3 \mathrm{~mm}$ apart in any direction) were identified, based on the assumption that such an extensive spatial coincidence of peak activation from the distal phalanx of three or more digits is not in line with the expected separate digit representations in BA $3 b$ and could therefore represent $a$ BOLD signal change due to a large blood vessel (Schweizer et al., 2010). In cases of such multiple digit foci the respective location was compared with the high-resolution angiograms to control for the presence of a vessel. If a vessel could be observed at the respective position, the area activated $($ at $q(F D R) \leq 0.05)$ upon stimulation of the two assumingly most distally represented fingers (e.g. D4 and D5 for positions close to the expected D1 representation) was marked and respective vertices were excluded from further analyses. 
2.2 Functional MRI reveals individual variations in the complete map of human digit phalanges in the primary somatosensory cortex

\section{Data analysis}

Stimulus-related activations were determined by a GLM analysis. For the functional localizer measurement, the main effect for the predictor of each of the 5 distal phalanges was calculated. For the intra-digit measurements, a GLM analysis for each digit was conducted calculating the main effects for the 4 predictors $p 1$ to $p 4$ (only $\mathrm{p} 1, \mathrm{p} 2$, and $\mathrm{p} 4$ for the thumb). All predictors were convolved with a two-gamma function (Friston et al., 1998) and voxels passing a t-value threshold corresponding to a false discovery rate of $\mathrm{q}(\mathrm{FDR})=0.05$ were considered significant. The resulting maps were projected onto the individual white-matter gray-matter surface reconstruction.

Further analysis was restricted to BA 3b, here defined as being located at the posterior wall of the central sulcus. Because no in-vivo marker for the identification of BA $3 b$ borders in individual subjects has been described, somatosensory mapping studies use a schematic approach to define the putative BA 3b/1 border (suggested by Geyer et al., 1999, used e.g. by Blankenburg et al., 2003, Nelson et al., 2008, and Stringer et al., 2011). We employed the junction of the vertical portion of the posterior bank of the central sulcus with the beginning of the curve of the crest of the postcentral gyrus as the putative border between BA $3 b$ and BA 1 (Moore et al., 2000). At the posterior wall of the central sulcus ending at the described junction, a putative BA 3b digit area was defined for each subject, based on the medial-tolateral extent of the $\mathrm{p} 1$ activation of the 5 digits measured in the first functional measurement, plus an addition of approximately $1 \mathrm{~cm}$ to either side of the most medial and lateral activation. The mesh-spanning vertices and their thresholded $t$-values within this BA $3 b$ digit area were exported to MATLAB.

For each phalanx of each finger, the coordinates of the mesh vertex with the highest t-value were determined as peak coordinates and used for further analysis. This peak-value approach is generally applied in somatosensory mapping studies (Kurth et al., 2000; Blankenburg et al., 2003; Nelson et al., 2008; Schweizer et al., 2008; Stringer et al., 2011) and has been shown to be the most reliable approximation to the position of the underlying neuronal activity measured with somatosensory event-related potentials in electroencephalography (Arthurs and Boniface, 2003). A description and comparison of BOLD activation overlap among the stimulation sites is beyond the scope of the present study. 
To provide a distance measure for comparison of the present data with other studies, mean Euclidean distances were calculated for each phalanx within a set of homologous phalanges, between the phalanx' peak voxel and the respective thumb's phalanx peak voxel:

$$
\sqrt{\left(x_{\text {digit,phal. }}-x_{D 1, \text { phal. }}\right)^{2}+\left(y_{\text {digit,phal. }}-y_{D 1, \text { phal. }}\right)^{2}+\left(z_{\text {digit,phal. }}-z_{D 1, \text { phal. }}\right)^{2}} \text {. }
$$

Analogously, for each phalanx within a digit, the mean intra-digit Euclidean distance between the phalanx peak voxel and the respective digit's first-phalanx peak voxel was calculated by

$$
\sqrt{\left(x_{\text {digit,phal. }}-x_{\text {digit }, p 1}\right)^{2}+\left(y_{\text {digit }, \text { hal. }}-y_{\text {digit }, p 1}\right)^{2}+\left(z_{\text {digit }, \text { hhal. }}-z_{\text {digit }, p 1}\right)^{2}} \text {. }
$$

To describe the map outline across subjects, an across-digit analysis was conducted. Activation peaks of all phalanges and bases were analyzed to determine direction vectors for homologous phalanges across all 5 digits (across 4 digits for p3). A principle component analysis (PCA) was applied to the peak-vertex coordinates of the phalanx representations in order to extract their main direction. For each subject and set of homologous phalanges the PC vector $\mathrm{N}=(\mathrm{Nx}, \mathrm{Ny}, \mathrm{Nz})$ was obtained (normalized to a length of 1). It describes the best linear fit and explains the largest amount of variance across the peak positions within a set of homologous phalanges (Fig. 2). To ensure that the $\mathrm{N}$ vector points into the general direction of D1 to $\mathrm{D} 5$, its sign was chosen such that the $\mathrm{N}$ coordinate with the largest absolute value had the same sign as the respective coordinate of the difference vector D5-D1 for across-digit analysis (i.e., if $\mathrm{Nx}>\mathrm{Ny}$ and $\mathrm{Nx}>\mathrm{Nz}$, then if $\mathrm{D} 5 x-\mathrm{D} 1 \mathrm{x}>0$, also $\mathrm{Nx}>0$ ). $\mathrm{N}$ coordinates were statistically tested at the group level to investigate a possible direction pattern across subjects. For each set of homologous phalanges (and digit bases) the respective $\mathrm{N}$ coordinates along each of the 3 ACPC axes were compared across subjects (one-sampled t-tests, two-tailed, at $p \leq 0.017$ corrected for testing along the 3 axes) to explore a possible across-digit direction consistency.

Building on that, a further set of analyses tested for a D1-to-D5 ordering of the homologous phalanges across the 5 digits. Peak vertices were transferred into an orthonormal coordinate system which included $\mathrm{N}$ as first base vector and thereby described the position of each phalanx representation along the line given by $\mathrm{N}$. 

phalanges in the primary somatosensory cortex

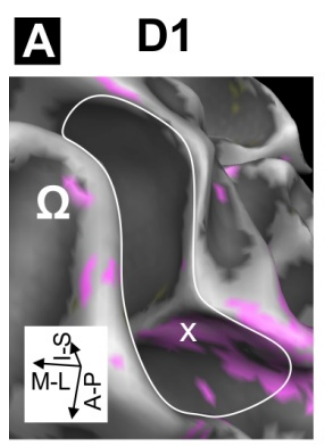

D2

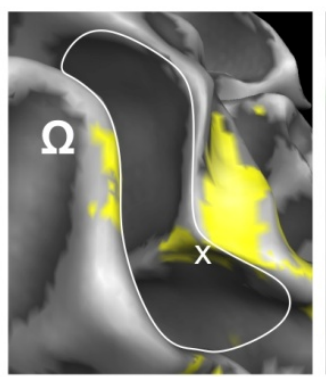

D3

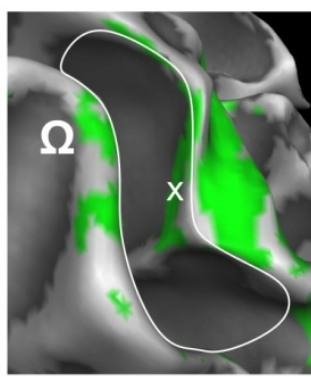

D4

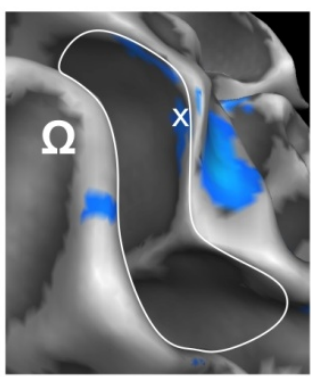

D5

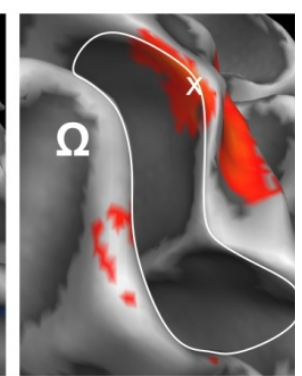

B

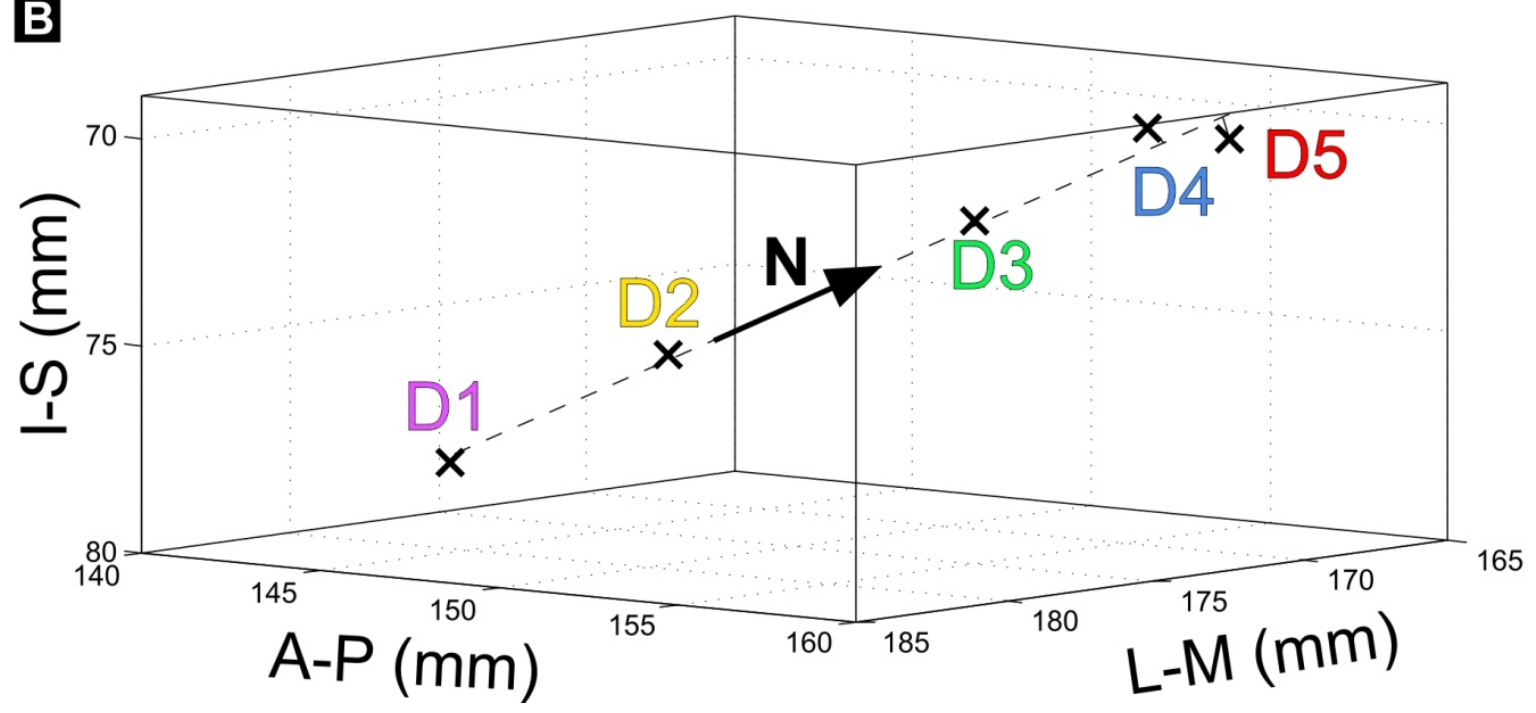

Figure 2. Direction vector $\mathbf{N}$ (for $\mathbf{p 1}$ of subject $\mathbf{S 2}$ ). (A) View from above onto the surface reconstruction of an individual left hemisphere, with the motor hand knob $(\Omega)$ in the foreground and the somatosensory BA $3 b$ hand area in the center $(A-P=$ anterior-posterior, $M-L=$ medial-lateral, $\mathrm{I}-\mathrm{S}=$ inferior-superior). The area used for peak-vertex analysis is delineated, peak vertices are marked by white crosses. From left to right, digit activations refer to D1 = magenta, D2 $=$ yellow, D3 = green, D4 = blue, and D5 = red. (B) Three-dimensional plot (in BrainVoyager $1 \mathrm{~mm}$ isotropic ACPC coordinates) of the 5 peak vertices and their best-fitting line (dashed) described by the direction vector $\mathrm{N}$. This subject shows somatotopy as the projections of the first phalanges onto $\mathrm{N}$ are sequential from D1 to D5.

Using this information, it could be assessed for each subject (and set of homologous phalanges), whether the peak vertices were ordered (from D1 to D5) according to the definition that neighboring phalanges yield similar or sequentially increasing coordinates along the PC vector and that not all peak vertices are identical. Binomial tests $(p \leq 0.05)$ across subjects examined whether more subjects than expected by chance showed an ordered D1-to-D5 pattern for homologous phalanges (across-digit ordering). The approach used the probabilities to find such an ordered pattern in individual subjects (i.e., probabilities 0.056 for $\mathrm{p} 1, \mathrm{p} 2$, and $\mathrm{p} 4$ and 0.189 for $\mathrm{p} 3$ ). 
For the intra-digit analysis, an analogous set of steps was performed across the three phalanges (two phalanges for the thumb). For each subject and digit, the normalized $P C$ vector $N$ was calculated, pointing in the general direction of $p 1$ to $p 3$ (from $\mathrm{p} 1$ to $\mathrm{p} 2$ for the thumb). At the group level, the $\mathrm{N}$ coordinates of a digit were compared to investigate a possible intra-digit direction consistency along any of the ACPC axes. For D2 to D5, a second analysis step assessed whether more subjects than expected by chance presented with ordered phalanges (from p1 to p3, intradigit ordering) along the PC vector (i.e., probability 0.5 for D2 to D5). 
2.2 Functional MRI reveals individual variations in the complete map of human digit phalanges in the primary somatosensory cortex

\section{Results}

Activation patterns from the functional localizer measurements showed the expected medial-to-lateral succession of digit representations from D5 to D1 along the posterior wall of the central sulcus. However, based on this data, 3 of 18 subjects had to be excluded, one due to no detectable activation in the assumed BA 3b, one due to the dominance of a large vessel parallel to the entire SI digit area, and one due to a rare variation of a central sulcus divided at the level of the hand knob (a complete pli de passage fronto-parietal moyen).

During tactile stimulation, subjects had to covertly count short randomly distributed interrupts of the tactile stimulation. Because there was on average only a $4 \%$ difference between the actual and reported numbers for each finger across subjects, it can be assumed that subjects were able to focus their attention towards the stimulation.

Comprehensive tactile stimulation of phalanges and bases could be accomplished in 15 subjects for D5, 14 subjects for D4, 14 subjects for D3, 14 subjects for D2, and 12 subjects for D1. Missing measurements resulted from difficulties in positioning the piezo-electric modules or from fatigue of the subjects due to the lengthy session. Significant activation of each stimulated phalanx and base could be observed within the expected digit area of BA $3 \mathrm{~b}$ in 13 out of 15 subjects (missing D4p3 and D3p4 activation in the remaining two subjects). After projection onto the white-matter gray-matter surface reconstruction and elimination of assumed large vessel contributions, significant BA 3b activations of all phalanges and the base were still observable for 11 subjects for D5, 11 subjects for D4, 12 subjects for D3, 14 subjects for D2, and 11 subjects for D1 (activation of all phalanges and the base of all digits observable in 7 subjects). Representations of the homologous phalanges and bases across digits were found in presumed BA $3 \mathrm{~b}$ (along the posterior wall of the central sulcus) (Fig. 3), spanning a D1-to-D5 distance of $16.7 \pm 4.2 \mathrm{~mm}$ for $\mathrm{p} 1,14.7 \pm 7.0 \mathrm{~mm}$ for $\mathrm{p} 2,18.0 \pm 3.2 \mathrm{~mm}$ for $\mathrm{p} 3$, and $11.2 \pm 8.4$ $\mathrm{mm}$ for $\mathrm{p} 4$ (see Table 1, upper part). Within digits (see Table 1, lower part), proximalto-distal phalanx distances of $4.8 \pm 2.6 \mathrm{~mm}$ for D1, $6.2 \pm 2.1 \mathrm{~mm}$ for D2, $6.6 \pm 5.2$ $\mathrm{mm}$ for D3, $5.0 \pm 3.1 \mathrm{~mm}$ for D4, and $3.4 \pm 2.3 \mathrm{~mm}$ for D5 were observed. The retest reliability across sessions (evaluated on 3 subjects) revealed a mean Euclidean 
Table 1. Euclidean distance spanned across digits and within digits. For each phalanx within a set of homologous phalanges (upper part of table), the mean across-digit Euclidean distance (mean \pm standard deviation) between the phalanx peak voxel and the respective thumb's phalanx peak voxel are stated (taking into account the 7 subjects showing mesh activation for each finger and phalanx). For each phalanx within a digit (lower part of table), the mean intra-digit Euclidean distance (mean \pm standard deviation) between the phalanx peak voxel and the respective digit's first-phalanx peak voxel are stated (same subjects as above).

\begin{tabular}{|c|c|c|c|c|c|c|c|c|c|c|c|c|}
\hline Distance to $\mathrm{D} 1 \mathrm{in} \mathrm{mm}$ & \multicolumn{3}{|c|}{ D2 } & \multicolumn{3}{|c|}{ D3 } & \multicolumn{3}{|c|}{ D4 } & \multicolumn{3}{|c|}{ D5 } \\
\hline $\mathrm{p} 1$ (to $\mathrm{p} 1$ of $\mathrm{D} 1$ ) & 6.8 & \pm & 2.1 & 11.4 & \pm & 3.2 & 15.1 & \pm & 5.7 & 16.7 & \pm & 4.2 \\
\hline $\mathrm{p} 2$ (to $\mathrm{p} 2$ of $\mathrm{D} 1$ ) & 4.6 & \pm & 3.0 & 9.2 & + & 5.1 & 13.3 & \pm & 7.0 & 14.7 & \pm & 7.0 \\
\hline p3 (to p2 of D1) & 8.1 & \pm & 2.3 & 12.8 & \pm & 3.8 & 15.9 & \pm & 4.1 & 18.0 & \pm & 3.2 \\
\hline p4 (to p4 of D1) & 6.9 & \pm & 7.6 & 12.6 & \pm & 9.7 & 8.2 & \pm & 7.0 & 11.2 & \pm & 8.4 \\
\hline Distance to $\mathrm{p} 1 \mathrm{in} \mathrm{mm}$ & & p2 & & & p3 & & & p4 & & & & \\
\hline $\mathrm{D} 1$ (to $\mathrm{p} 1$ of $\mathrm{D} 1$ ) & 4.8 & \pm & 2.6 & & & & 14.4 & \pm & 8.7 & & & \\
\hline $\mathrm{D} 2$ (to $\mathrm{p} 1$ of $\mathrm{D} 2$ ) & 4.0 & \pm & 2.8 & 6.2 & \pm & 2.1 & 9.2 & \pm & 4.2 & & & \\
\hline D3 (to $p 1$ of D3) & 2.9 & \pm & 2.7 & 6.6 & \pm & 5.2 & 8.8 & \pm & 5.6 & & & \\
\hline D4 (to $\mathrm{p} 1$ of $\mathrm{D} 4$ ) & 2.9 & \pm & 2.9 & 5.0 & \pm & 3.1 & 6.8 & \pm & 3.0 & & & \\
\hline D5 (to $\mathrm{p} 1$ of $\mathrm{D} 5$ ) & 2.3 & \pm & 2.4 & 3.4 & \pm & 2.3 & 5.4 & \pm & 5.0 & & & \\
\hline
\end{tabular}

peak-vertex difference of $2.9 \pm 3.6 \mathrm{~mm}$ (averaged across subjects and stimulation sites).

Visual inspection confirmed the expected digit succession for the first phalanx, progressing from medial-posterior-superior (D5) to lateral-anterior-inferior (D1). A similar pattern was observed for the second and third phalanx (Fig. 3), in agreement with the general finding of strong overlap and closely located peak values between p1-to-p3 (and sometimes p4) phalanx activations within subjects. The succession from D1 to D5 for peak vertices of all phalanges is illustrated in Fig. 4A (also pointed to by the increasing row values in Table 1, upper part). The corresponding base representations were more variable (Fig. 4B), ranging from the vicinity of (or even overlap with) the respective phalanx activations (Fig. 3, subject S5) to positions lateral to D1 or more commonly medial to D5 (Fig. 3, subject S7). Within each digit, increasing Euclidean distances from p1 to p4 were observed (row values in Table 1, lower part), although visual inspection pointed to a p1-to-p4 succession only in D5 and perhaps D4. 
2.2 Functional MRI reveals individual variations in the complete map of human digit phalanges in the primary somatosensory cortex

Figure 3. Across-digit representations of phalanges and bases (subjects S3, S5, and S7). View from above onto the inflated surface reconstruction of individual left hemispheres with the motor hand knob $(\Omega)$ in the foreground and the somatosensory BA $3 \mathrm{~b}$ hand area in the center (convex areas $=$ light grey, concave areas $=$ dark grey, putative vessels $=$ white $A-P=$ anterior-posterior, $\mathrm{M}-\mathrm{L}=$ medial-lateral). The area used for peak-vertex analysis is delineated. Each column shows the activations for homologous phalanges p1, p2, and p3 as well as digit bases p4. D1 = magenta, D2 = yellow, D3 = green, D4 = blue, and D5 = red.

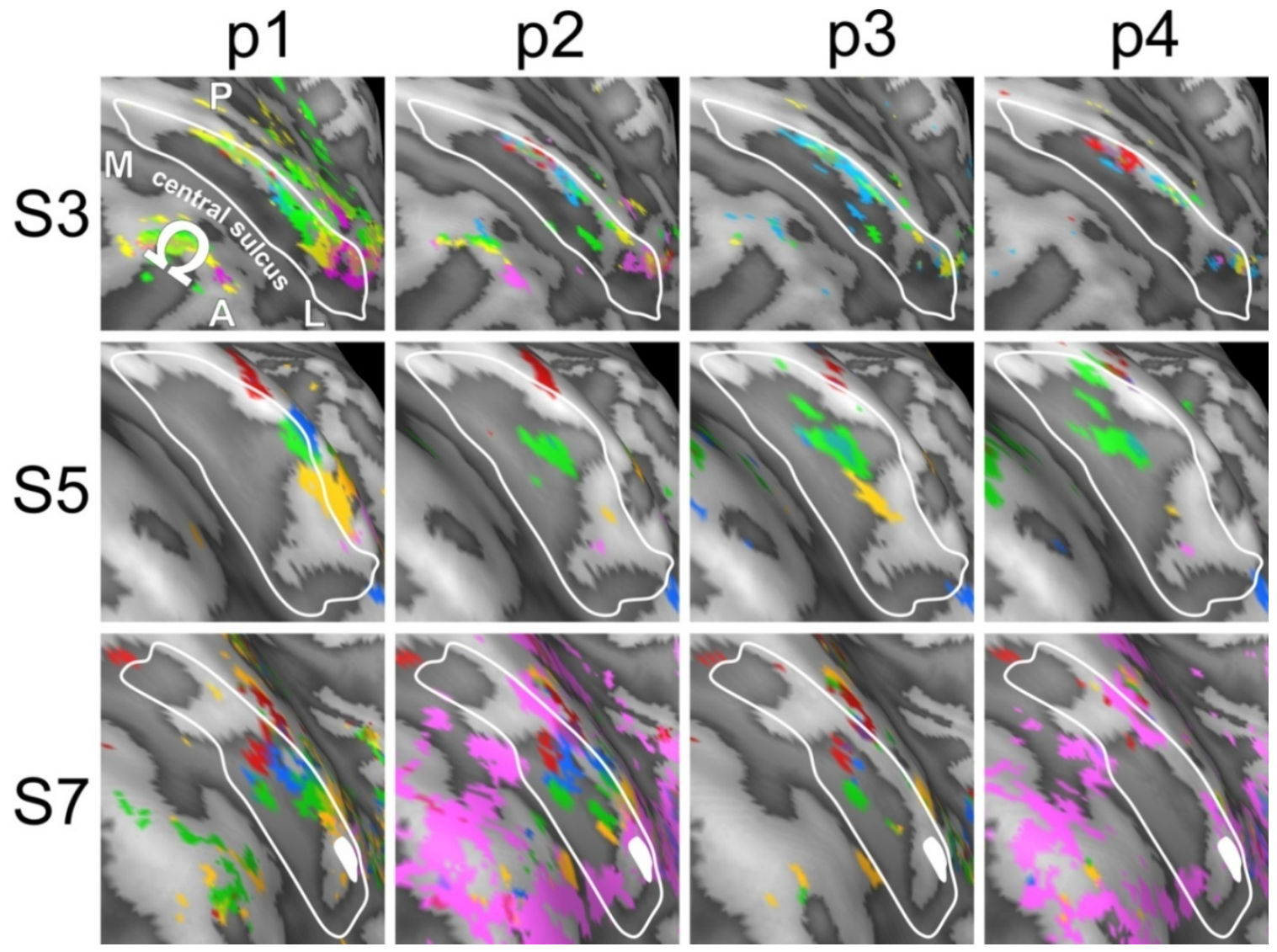

Figure 4. Organization of digit area in BA 3b. For each of the 7 subjects that presented with significant activation for all phalanges and bases, all peak vertices were normalized by the respective subject's mean value across phalanges (but not bases; $A-P=$ anterior-posterior, $M-L=$ mediallateral, I-S = inferior-superior, D1 = magenta, D2 = yellow, D3 = green, D4 = blue, D5 = red; all units in $1 \mathrm{~mm})$. (A) Normalized peak-vertex coordinates for p1 $(=1)$, p2 $(=2)$, and p3 $(=3)$. (B) Normalized peak-vertex coordinates for $\mathrm{p} 4(=4)$.
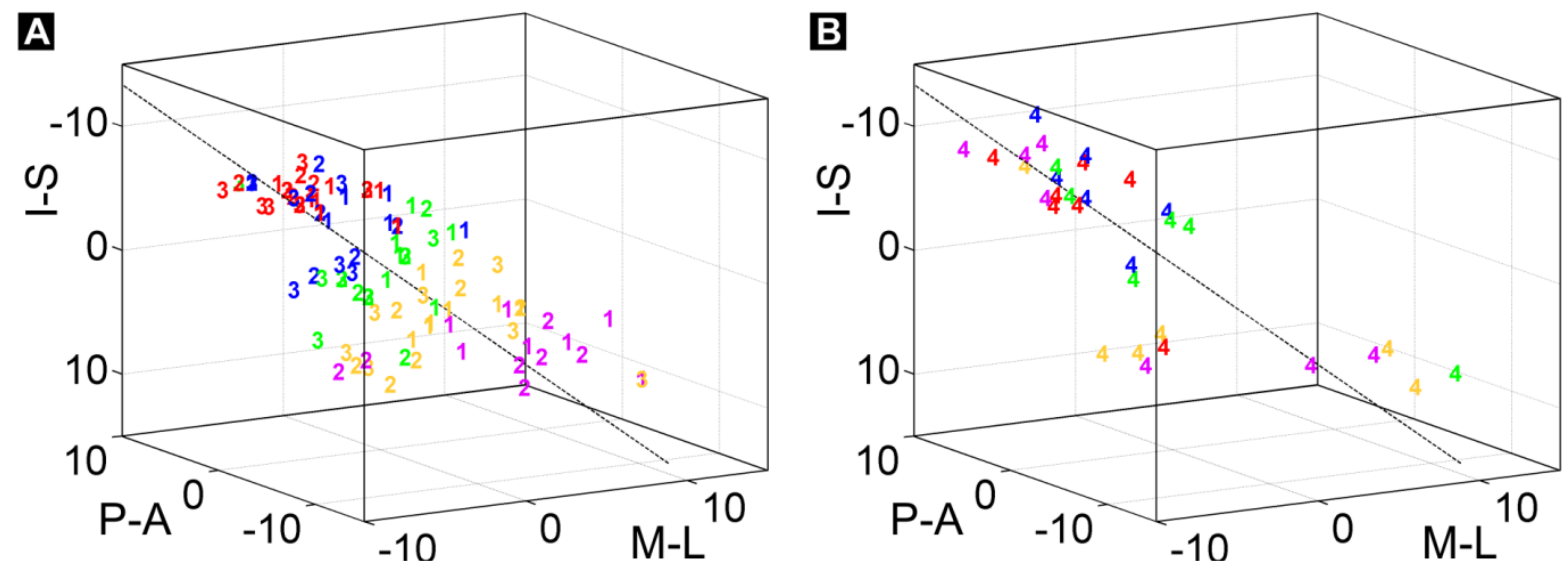


\section{Across-digit direction consistency}

The general direction of the distribution of homologous phalanges $\mathrm{p} 1, \mathrm{p} 2$, and p3 or bases p4 across digits was determined in each subject showing significant activations in all digits (Table 2). Individual direction vectors were similar across subjects, leading to large average direction vectors for p1, p2, and p3 (Fig. 5A). Across-subjects t-tests revealed significant across-digit direction consistency along all 3 ACPC axes, not only for the distal phalanges $\mathrm{p} 1$ but also for the second and third phalanges $\mathrm{p} 2$ and $\mathrm{p} 3$ (Table 2). The average direction vectors were similarly oriented for the three phalanges, all pointing from medial-posterior-superior to lateral-anterior-inferior. For the bases p4, such an across-digit direction consistency could not be observed, as the $\mathrm{N}$ vectors of different subjects pointed to different directions (Fig. 5A).

\section{Across-digit ordering}

For each set of homologous phalanges (or bases) and subject, it was assessed whether the projections of the respective peak vertices onto their bestfitting line (described by the $N$ vector) were ordered from D1 to D5. The expected D1-to-D5 ordering between homologous phalanges (or bases) could be shown and statistically confirmed not only for the first but also for the second and third phalanges (Fig. 5B). In the 5 out of 25 individual cases in which the expected acrossdigit ordering was not observed, the swap always occurred between neighboring digits: three times between D4/D5, once between D3/D4, and once between D1/D2. For the base, on the contrary, no across-digit ordering could be observed, as only 2 out of 9 subjects showed D1-to-D5 ordering.

Table 2. Across-digit direction consistency. Average $\mathrm{N}$ vectors (calculated across D1-to-D5 peak vertices), respective coordinates and standard deviations (SD) as well as Bonferroni-corrected $\mathrm{p}$-values are stated for phalanges $\mathrm{p} 1$ to $\mathrm{p} 3$ and digit bases $\mathrm{p} 4$ along the medial-lateral $(\mathrm{M}-\mathrm{L})$, anterior-posterior (A-P), and inferior-superior (I-S) ACPC axes, respectively. * $p \leq 0.05$, Bonferroni corrected

\begin{tabular}{|c|c|c|c|c|c|c|c|c|c|c|c|c|}
\hline Phalanx & p1 & & & $\mathrm{p} 2$ & & & p3 & & & p4 & & \\
\hline & M-L & A-P & I-S & M-L & A-P & I-S & M-L & A-P & I-S & M-L & $A-P$ & I-S \\
\hline $\begin{array}{l}\text { Mean N } \\
\text { SD }\end{array}$ & $\begin{array}{r}-0.39 \\
\pm 0.23\end{array}$ & $\begin{array}{r}0.47 \\
\pm 0.34\end{array}$ & $\begin{array}{r}0.68 \\
\pm 0.14\end{array}$ & $\begin{array}{r}-0.28 \\
\pm 0.24\end{array}$ & $\begin{array}{r}0.52 \\
\pm 0.28\end{array}$ & $\begin{array}{r}0.70 \\
\pm 0.22\end{array}$ & $\begin{array}{r}-0.34 \\
\pm 0.27\end{array}$ & $\begin{array}{r}0.43 \\
\pm 0.36\end{array}$ & $\begin{array}{r}0.65 \\
\pm 0.35\end{array}$ & $\begin{array}{r}0.11 \\
\pm 0.51\end{array}$ & $\begin{array}{l}-0.06 \\
\pm 0.54\end{array}$ & $\begin{array}{r}0.01 \\
\pm 0.74\end{array}$ \\
\hline $\mathrm{p}$-value & $0.003^{*}$ & $0.010^{*}$ & $<0.001^{*}$ & $0.039^{*}$ & $0.003^{*}$ & $<0.001^{*}$ & $0.025^{*}$ & $0.036^{*}$ & $0.004^{*}$ & 1 & 1 & 1 \\
\hline
\end{tabular}


2.2 Functional MRI reveals individual variations in the complete map of human digit phalanges in the primary somatosensory cortex

Figure 5. Across-digit direction consistency and ordering. (A) Across-digit direction vectors for p1 to p4 (black arrows = individual direction vectors, red arrows $=$ mean values averaged across subjects; $\mathrm{A}-\mathrm{P}=$ anterior-posterior, $\mathrm{M}-\mathrm{L}=$ medial-lateral, $\mathrm{I}-\mathrm{S}=$ inferior-superior). Across-digit direction consistency is indicated by an asterisk and respective Bonferroni-corrected p-values. (B) Across-digit ordering as given by the number of subjects showing (black) or not showing (grey) ordered activations from D1 to D5 along the individual $\mathrm{N}$ vectors. The red lines illustrate the minimal number of ordered subjects needed to observe across-digit ordering. Phalanges showing ordering are indicated by an asterisk and respective $p$-values.
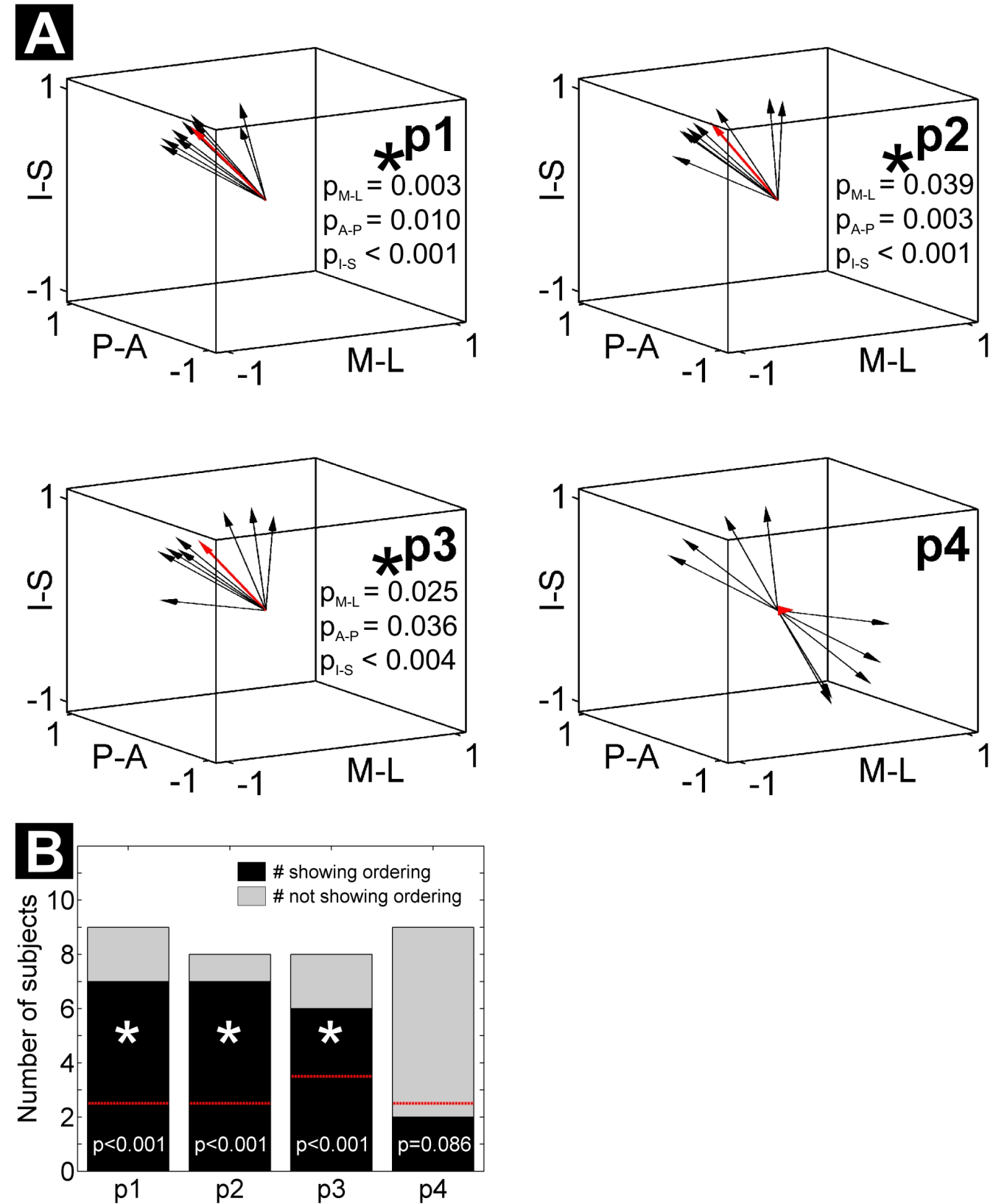

\section{Intra-digit direction consistency and ordering}

Visual inspection of the respective activation patterns revealed no clear succession between phalanx activations except for a general medial-to-lateral p4-top1 succession in D5 (Fig. 6). 
A direction vector $\mathrm{N}$ was calculated for those subjects that showed significant activation for all phalanges of a digit (Fig. 7A). Intra-digit direction consistency was found only for some digits across subjects (Table 3). D5 exhibited a significant intradigit direction consistency along the medial-lateral and anterior-posterior axes, with the $\mathrm{N}$ vector generally pointing into medial-posterior direction. For D4, intra-digit direction consistency was observed from anterior to posterior. The direction vectors of D1, D2, and D3 did not show any consistency across subjects.

For each digit (except for D1) and subject it was tested whether the projections of the peak vertices onto their PC vector were ordered from $\mathrm{p} 1$ to $\mathrm{p} 3$. The number of subjects exhibiting ordered patterns is shown in Figure 7B. For D5, significantly more subjects ( 9 out of 11) than expected by chance exhibited an ordered phalanx representation. For D4, significance was missed by only a single subject, while no intra-digit ordering could be observed for D3 and D2.

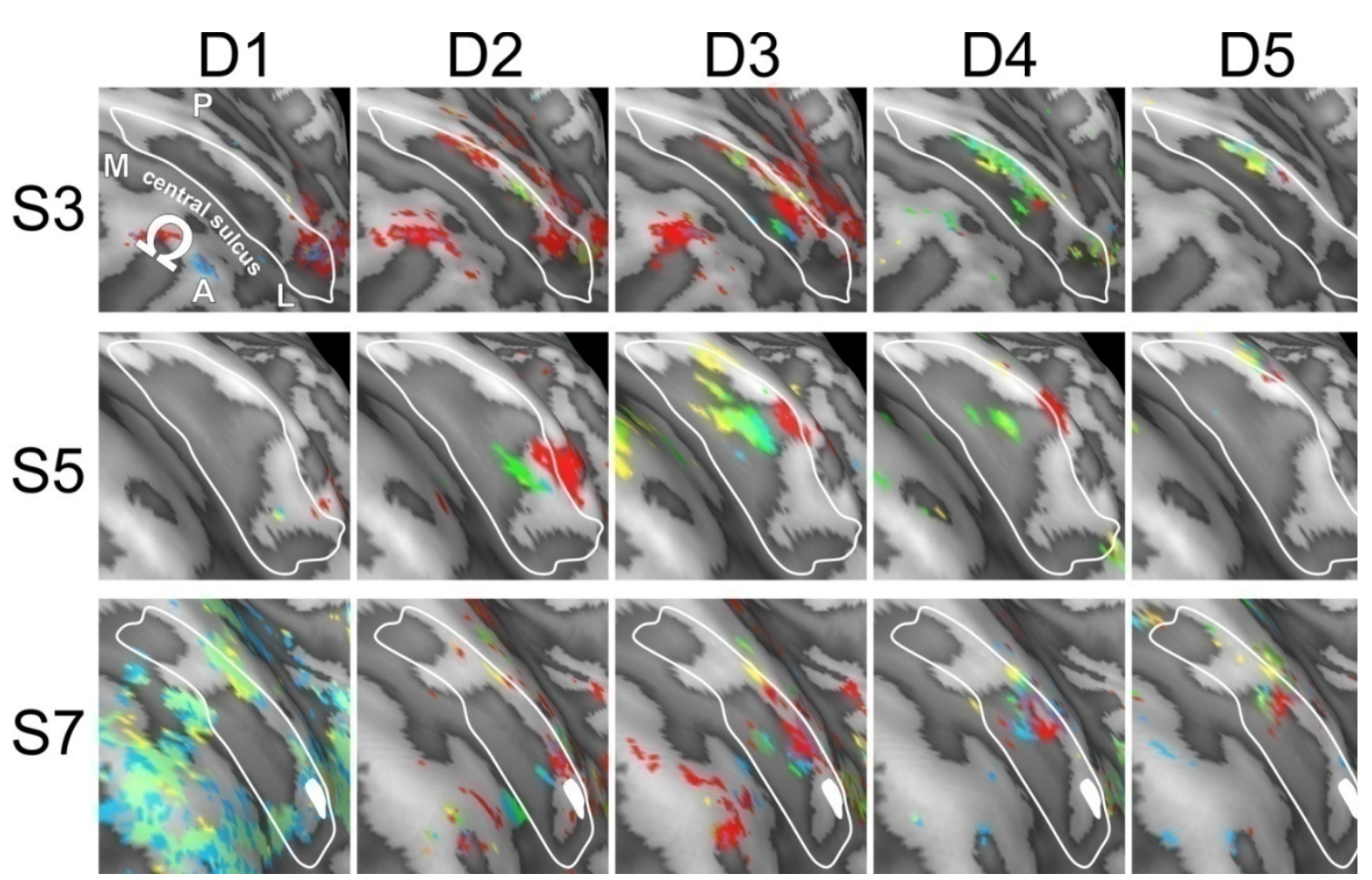

Figure 6. Intra-digit representations of phalanges and bases (subjects S3, S5, and S7). View from above onto the inflated surface reconstruction of the left hemisphere with the motor hand knob $(\Omega)$ in the foreground and the somatosensory BA 3b hand area in the center (convex areas = light grey, concave areas $=$ dark grey, putative vessels $=$ white, $A-P=$ anterior-posterior, $M-L=$ medial-lateral). The area used for peak-vertex analysis is delineated. Each column shows the activations for phalanges p1 (= red), p2 (= blue), and p3 (= green) as well as base p4 (= yellow) of a single digit (D1 to D5). 
2.2 Functional MRI reveals individual variations in the complete map of human digit phalanges in the primary somatosensory cortex

Table 3. Average $\mathbf{N}$ vectors (calculated across the $\mathrm{p} 1$-to-p4 peak vertices), respective coordinates and standard deviations (SD), and Bonferroni-corrected p-values for digits D1 to D5 along the medial-lateral (M-L), anterior-posterior (A-P), and inferior-superior (I-S) ACPC axis, respectively.

${ }^{*} p \leq 0.05$, Bonferroni corrected

\begin{tabular}{|c|c|c|c|c|c|c|c|c|c|c|c|c|c|c|c|}
\hline Digit & \multicolumn{3}{|c|}{ D1 } & \multicolumn{3}{|c|}{ D2 } & \multicolumn{3}{|c|}{ D3 } & \multicolumn{3}{|c|}{ D4 } & \multicolumn{3}{|c|}{ D5 } \\
\hline & $M-L$ & A-P & I-S & M-L & $A-P$ & $1-3$ & IVI & $A-P$ & 1. & $M$ & $A-P$ & ט-ו & $\mathrm{IVI}-\mathrm{L}$ & $A-P$ & I-S \\
\hline Mean & -0.27 & 0.16 & -0.25 & -0.12 & 0.06 & -0.17 & -0.10 & 0.21 & -0.24 & -0.30 & 0.37 & -0.22 & -0.43 & 0.46 & 0.23 \\
\hline & \pm 0.59 & \pm 0.40 & \pm 0.64 & \pm 0.56 & \pm 0.55 & \pm 0.64 & \pm 0.50 & \pm 0.58 & \pm 0.62 & \pm 0.46 & \pm 0.33 & \pm 0.69 & \pm 0.39 & \pm 0.34 & \pm 0.58 \\
\hline$p$-value & 0.462 & 0.670 & 0.681 & 1 & 1 & 1 & 1 & 0.740 & 0.621 & 0.169 & $0.011^{*}$ & 0.963 & $0.014^{*}$ & $0.003^{*}$ & 0.681 \\
\hline
\end{tabular}

Figure 7. Intra-digit direction consistency and ordering. (A) Intra-digit direction vectors for D1 to D5 (black arrows = individual direction vectors, red arrows = mean values averaged across subjects; $\mathrm{A}-\mathrm{P}=$ anterior-posterior, $\mathrm{M}-\mathrm{L}=$ medial-lateral, $\mathrm{I}-\mathrm{S}=$ inferior-superior). Intra-digit direction consistency is indicated by an asterisk and respective Bonferroni-corrected p-values. (B) Intra-digit ordering as given by the number of subjects showing (black) or not showing (grey) ordered activations from $\mathrm{p} 1$ to p3 along the individual $\mathrm{N}$ vectors. The red lines illustrate the minimal number of ordered subjects needed to observe intra-digit ordering. Digits showing ordering are indicated by an asterisk and respective $p$-values.
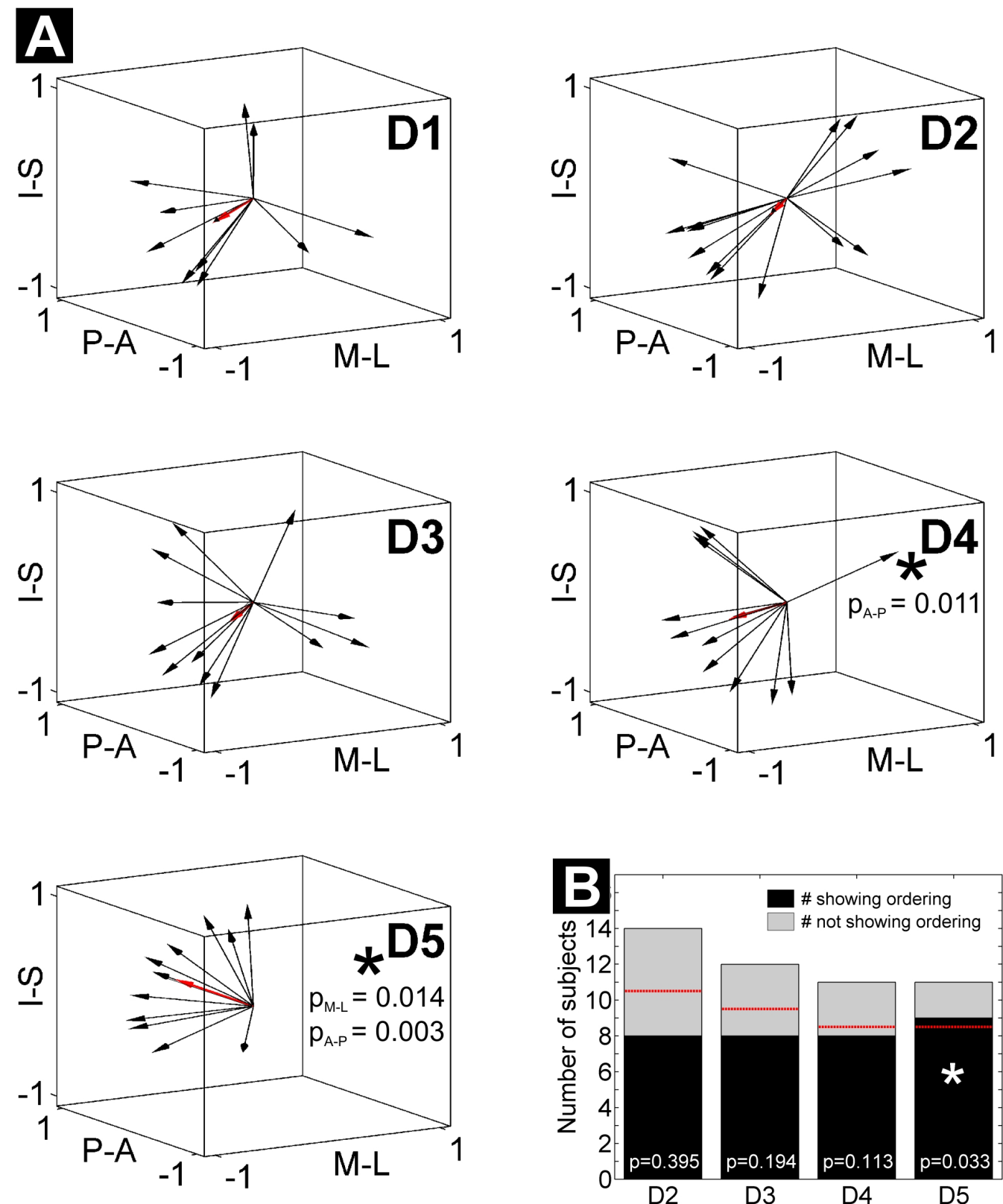


\section{Discussion}

The goal of the present study was to delineate the complete map of phalanx and base representations of all 5 digits in BA $3 \mathrm{~b}$ in the individual human primary somatosensory cortex. The analysis of the fMRI activation patterns and their ordering along a principal-component direction revealed a divergent picture of topography in across-digit and intra-digit maps.

Across digits, the observed succession of first-phalanx representations from D5 to D1 along the general course of the central sulcus (from medial to lateral, superior to inferior, and posterior to anterior) confirms previous fMRI studies describing the layout of the human digit area in BA 3b (Maldijan et al., 1999; Kurth et al., 2000; Nelson et al., 2008; Schweizer et al., 2008; Sanchez-Panchuelo et al., 2010). It also demonstrates that the newly applied analysis provides a valid and sensitive strategy to capture and statistically compare the layout of maps from individual subjects as well as to extract general features.

The succession seen in the distal phalanges is also present in the more proximal phalanges, as both p2 and p3 similarly exhibited across-digit directions along the central sulcus and showed the corresponding succession from D5 to D1 for p2 and from D5 to D2 for p3. These findings are analogous to the digit organization seen in electrophysiological mapping studies of squirrel, owl, and macaque monkeys (Sur et al., 1982; Merzenich et al., 1978; Iwamura et al., 1983) and can be described as the main axis of digit representation along the course of the central sulcus in analogy to the eccentricity axis along the calcarine sulcus of the primary visual cortex (Sereno et al., 1994).

For the digit bases (p4) we could not detect a consistent pattern of cortical representations across subjects. p4 peak representations were located close to the activations of the respective digit's phalanges as well as medial to the digit area of D5 and lateral to that of D1. This resembles the maps of owl monkeys with p4 located posterior to p3 of the respective digit (Merzenich et al., 1978) as well as aspects found in macaques with p4 representations medial-posterior to D5 for D5 and D4 and lateral-posterior to D1 for D3 and D2 (Nelson et al., 1980; Kaas et al., 1979; Iwamura et al., 1983). 
2.2 Functional MRI reveals individual variations in the complete map of human digit phalanges in the primary somatosensory cortex

The intra-digit analysis showed a much lower degree of consistency across subjects than the across-digit analysis. Only digits D5 and D4 presented with a common axis across subjects along which phalanx activations were distributed and only D5 exhibited an ordering from $\mathrm{p} 1$ to $\mathrm{p} 3$ (for D4, significance was missed by one subject). These observations confirm and extend the results of our previous study (Schweisfurth et al., 2011), demonstrating a consistent arrangement between $\mathrm{p} 1$ and p4 for D5 but not for D2 across subjects. The common direction along which the D5 representations were ordered pointed from lateral $(p 1)$ to medial (p4) in the previous and lateral-anterior ( 1 1) to medial-posterior (p3) in the present study. Both results indicate an intra-digit ordering along the general course of the central sulcus, not perpendicular to it as in anesthetized owl and macaque monkeys (Merzenich et al, 1978; Nelson et al., 1980). This finding may be due to the relatively small extent of the D5 digit area (Iwamura et al., 1983) and the small distance between the BA 3b D5p1 representation and the putative BA 3b/1 border (Schweisfurth et al., 2011) that possibly drive the representations of $\mathrm{p} 2$ and $\mathrm{p} 3$ into the medial direction. The successive increase in Euclidean distance between p1 and p3 representations across D5 through D1 is in accordance to the maps of awake macaques (Iwamura et al., 1983) and can also be estimated from the D5-through-D1 p1 coordinates in other mapping studies (Kurth et al., 2000; Nelson et al., 2008).

For D3, D2, and D1 no significant intra-digit direction consistency could be detected, as the directions along which the phalanges were arranged differed substantially between subjects. In addition, no significant intra-digit ordering between phalanges along the individual direction vectors was found for D3 or D2. These results are in contrast to studies in anesthetized monkeys (Merzenich et al., 1978; Nelson et al., 1980) and two single-digit fMRI studies (Blankenburg et al., 2003; Sanchez-Panchuelo et al., 2012). Blankenburg and colleagues reported a rostral(p1)-to-caudal(p4) succession of phalanx representations of D3 across BA 3b despite coarser spatial resolution and as a result of a group analysis, pooling the BOLD activations of 8 subjects. Factors contributing to the divergence of their result to our present study may be our increased spatial resolution, our higher number of subjects as well as our analysis of individual maps. In the study of SanchezPanchuelo and colleagues (2012) the authors identified map reversals of the p3-top1 representation of $\mathrm{D} 2$ in $\mathrm{SI}$, although no objective measure is given to justify the 
identification of the mirror-reversal positions. The across-subject variation in the general directions of the mirror reversal of the maps as well as in the implied ordering of the phalanges seems to be comparable to the variations in direction and ordering of our intra-digit representations of D2. The divergent results for anesthetized monkeys also have to be put into perspective by the awake-macaque study reporting that the narrow zone of proximal-phalanx (p2/p3) representations located towards the $\mathrm{BA} 3 \mathrm{~b} / 1$ border also contained receptive fields of distal phalanges (mainly of D3). This intermittent spread of $p 1$ representations into the proximal-phalanx representation area could explain the divergent ordering and directions of phalanx representations within D3 and D2 among our subjects.

Additionally, specific methodological considerations have to be taken into account in the interpretation of our data. Outlining the complete map of all phalanges of all digits in one session in order to omit the influence from across-session variations, comes with the price of less averages per stimulation site and a slight decrease in the across-session reliability compared to our previous study with larger number of trials per stimulation site (Schweisfurth et al., 2011). Whether biological variability of the somatosensory map on the phalanx level additionally adds to the retest variation, remains to be elucidated. Another possible reason of the observed digit differences might stem from individual variations of the central sulcus at the spatially-complex hand knob, potentially affecting the principle-component vectors of D2 and D1 more than those of D4 and D5. However, this could largely be ruled out by thorough retrospective inspection of intra-digit direction vectors, assuring their reliable representation of the $\mathrm{fMRI}$ activations.

In conclusion, the evidence for the proposed second perpendicular axis representing the intra-digit somatotopy in a rostral-to-caudal ordered fashion is less clear due to substantial variations across individual subjects concerning both the direction and ordering of this intra-digit axis. As studied here the map of this axis represents the peripheral sensory receptor sheet of the digit: It can be used in various different ways and in various positions, and therefore is apparently not as precisely laid out as the phase-angle map in the visual system, getting input from a stable, accurately-defined visual field. Merzenich and colleagues (1987) who specifically reported the aspect of individual variability in certain aspects of the across-digit topography of a greater number of owl and squirrel monkeys proposed 
2.2 Functional MRI reveals individual variations in the complete map of human digit phalanges in the primary somatosensory cortex

that specific details of the cortical maps might be the consequences of individual differences in lifelong hand use. Later studies in macaques and humans indeed demonstrated the variability of cortical somatosensory maps not only in response to amputation (Merzenich et al., 1984; Flor et al., 1995) but also in the intact adult individual due to changes in digit usage (Jenkins et al., 1990; Recanzone et al., 1992; Elbert et al., 1995; Sterr et al., 1998; Braun et al., 2000). Based on these findings we hypothesize that the specific layout of the individual intra-digit maps in D3, D2, and D1 reflects the plasticity of the primary somatosensory cortex in response to individual differences in the usage of the digits. More specifically, the difference in ordering and direction consistency of D5 (and partly D4) versus D3, D2, and D1 might reflect the degree of individualization in which the digits of the right, dominant human hand are used or trained. Conversely, daily usage as well as clinical and rehabilitation experience show that D5 and D4 of the dominant hand mainly act as supporting digits that are potentially used in a very similar manner across subjects. In the resulting tactile cortical maps this may lead to a more ordered intra-digit arrangement which is consistent across subjects. In contrast, the usage of D3, D2, and D1 involves the complex manipulation of objects, the performance of pinch grips, and the handling of tools like pens, cutlery, or other utensils that are prone to a much more individualized, variable, and flexible strategy, which might be ultimately reflected in individualized cortical representations and intra-digit maps of these digits. 


\section{Acknowledgements}

The current work was supported by the International Max Planck Research School MSc/PhD/MD-PhD Neuroscience Program (to M.A.S.) of the Göttingen Graduate School of Neurosciences and Molecular Biosciences (GGNB) in Göttingen, Germany, and the graduate school Neurosenses (to M.A.S.) in Göttingen and Oldenburg, Germany. We would like to thank Leeanne M. Carey for sharing helpful scientific and clinical insights informing our discussion of the usage of the hand. 
2.2 Functional MRI reveals individual variations in the complete map of human digit phalanges in the primary somatosensory cortex

\section{References}

Allard T, Clark SA, Jenkins WM, Merzenich MM (1991) Reorganization of somatosensory area 3b representations in adult owl monkeys after digital syndactyly. Journal of Neurophysiology 66: 1048-1058.

Arthurs OJ, Boniface SJ (2003) What aspect of the fMRI BOLD signal best reflects the underlying electrophysiology in human somatosensory cortex? Clinical Neurophysiology 114: 1203-1209.

Blankenburg F, Ruben J, Meyer R, Schwiemann J, Villringer A (2003) Evidence for a rostral-to-caudal somatotopic organization in human primary somatosensory cortex with mirror-reversal in areas $3 b$ and 1. Cerebral Cortex 13: 987-993.

Braun C, Schweizer R, Elbert T, Birbaumer N, Taub E (2000) Differential activation in somatosensory cortex for different discrimination tasks. Journal of Neuroscience 20: 446-450.

Cheng K, Waggoner RA, Tanaka K (2001) Human Ocular Dominance Columns as Revealed by HighField Functional Magnetic Resonance Imaging. Neuron 32: 359-374.

Elbert T, Pantev C, Wienbruch C, Rockstroh B, Taub E (1995) Increased cortical representation of the fingers of the left hand in string players. Science 270: 305-307.

Flor H, Elbert T, Knecht S, Wienbruch C, Pantev C, Birbaumers N, Larbig W, Taub E (1995) Phantom-limb pain as a perceptual correlate of cortical reorganization following arm amputation. Nature 375: 482-484.

Friston KJ, Fletcher P, Josephs O, Holmes A, Rugg MD, Turner R (1998) Event-related fMRI: Characterizing differential responses. Neurolmage 7: 30-40.

Geyer S, Schleicher A, Zilles K (1999) Areas 3a, 3b, and 1 of Human Primary Somatosensory Cortex: 1. Microstructural Organization and Interindividual Variability. Neurolmage 10: 63-83.

Goebel R, Esposito F, Formisano E (2006) Analysis of functional image analysis contest (FIAC) data with brainvoyager QX: From single-subject to cortically aligned group general linear model analysis and self-organizing group independent component analysis. Human Brain Mapping 27: 392-401.

Iwamura Y, Tanaka M, Sakamoto M, Hikosaka O (1983) Functional subdivisions representing different finger regions in area 3 of the first somatosensory cortex of the conscious monkey. Experimental Brain Research 51: 315-326.

Jenkins WM, Merzenich MM, Ochs MT, Allard T, Guicrobles E (1990) Functional reorganization of primary somatosensory cortex in adult owl monkeys after behaviorally controlled tactile stimulation. Journal of Neurophysiology 63: 82-104.

Jenkinson M, Smith SM (2001) A global optimisation method for robust affine registration of brain images. Medical Image Analysis 5: 143-156.

Johansson RS, Vallbo AB (1979) Tactile sensibility in the human hand: relative and absolute densities of four types of mechanoreceptive units in glabrous skin. The Journal of Physiology 286: 283-300.

Kaas JH, Nelson RJ, Sur M, Lin CS, Merzenich MM (1979) Multiple representations of the body within the primary somatosensory cortex of primates. Science 204: 521-523.

Kaas JH (1997) Topographic maps are fundamental to sensory processing. Brain Research Bulletin 44: 107-112. 
Kurth R, Villringer K, Curio G, Wolf KJ, Krause T, Repenthin J, Schwiemann J, Deuchert M, Villringer A (2000) fMRI shows multiple somatotopic digit representations in human primary somatosensory cortex. NeuroReport 11: 1487 - 1491.

Maldjian JA, Gottschalk A, Patel RS, Detre JA, Alsop DC (1999) The sensory somatotopic map of the human hand demonstrated at 4 Tesla. Neurolmage 10: 55-62.

Merzenich MM, Kaas JH, Sur M, Lin C-S (1978) Double representation of the body surface within cytoarchitectonic area $3 \mathrm{~b}$ and 1 in $\mathrm{SI}$ in the owl monkey (aotus trivirgatus). The Journal of Comparative Neurology 181: 41-73.

Merzenich MM, Nelson RJ, Stryker MP, Cynader MS, Schoppmann A, Zook JM (1984) Somatosensory cortical map changes following digit amputation in adult monkeys. Journal of Comparative Neurology 224: 591-605.

Merzenich MM, Nelson RJ, Kaas JH, Stryker MP, Jenkins WM, Zook JM, Cynader MS, Schoppmann A (1987) Variability in hand surface representations in areas $3 \mathrm{~b}$ and 1 in adult owl and squirrel monkeys. The Journal of Comparative Neurology 258: 281-296.

Moore CI, Stern CE, Corkin S, Fischl B, Gray AC, Rosen BR, Dale AM (2000) Segregation of somatosensory activation in the human rolandic cortex using fMRI. Journal of Neurophysiology 84: 558-569.

Nelson RJ, Sur M, Felleman DJ, Kaas JH (1980) Representations of the body surface in postcentral parietal cortex of Macaca fascicularis. The Journal of Comparative Neurology 192: 611-643.

Nelson AJ, Chen R (2008) Digit somatotopy within cortical areas of the postcentral gyrus in humans. Cerebral Cortex 18: 2341-2351.

Oldfield RC (1971) The assessment and analysis of handedness: The Edinburgh inventory. Neuropsychologia 9: 97-113.

Penfield W, Boldrey E (1937) Somatic motor and sensory representation in the cerebral cortex of man as studied by electrical stimulation. Brain 60: 389-443.

Penfield W, Rasmussen T (1950) The cerebral cortex of man: A clinical study of localization of function. New York: Macmillan.

Polimeni JR, Fischl B, Greve DN, Wald LL (2010) Laminar analysis of 7T BOLD using an imposed spatial activation pattern in human V1. Neurolmage 52: 1334-1346.

Pons TP, Preston EG, Ommaya AK, Jon HK, Taub E, Mishkin M (1991) Massive cortical reorganization after sensory deafferentation in adult macaques. Science 252: 1857-1860.

Recanzone GH, Merzenich MM, Jenkins WM, Grajski KA, Dinse HR (1992) Topographic reorganization of the hand representation in cortical area $3 \mathrm{~b}$ of owl monkeys trained in a frequency-discrimination task. Journal of Neurophysiology 67: 1031-1056.

Sanchez-Panchuelo RM, Francis S, Bowtell R, Schluppeck D (2010) Mapping human somatosensory cortex in individual subjects with 7T functional MRI. Journal of Neurophysiology 103: 25442556.

Sanchez-Panchuelo RM, Besle J, Beckett A, Bowtell R, Schluppeck D, Francis S (2012) Within-digit functional parcellation of Brodmann areas of the human primary somatosensory cortex using functional magnetic resonance imaging at 7 Tesla. The Journal of Neuroscience 32: 1581515822.

Schweisfurth MA, Schweizer R, Frahm J (2011) Functional MRI indicates consistent intra-digit topographic maps in the little but not the index finger within the human primary somatosensory cortex. Neurolmage 56: 2138-2143. 
2.2 Functional MRI reveals individual variations in the complete map of human digit phalanges in the primary somatosensory cortex

Schweizer R, Voit D, Frahm J (2008) Finger representations in human primary somatosensory cortex as revealed by high-resolution functional MRI of tactile stimulation. Neurolmage 42: 28-35.

Sereno MI, McDonald CT, Allman JM (1994) Analysis of retinotopic maps in extrastriate cortex. Cerebral Cortex 4: 601-620.

Shmuel A, Yacoub E, Chaimow D, Logothetis NK, Ugurbil K (2007) Spatio-temporal point-spread function of fMRI signal in human gray matter at 7 Tesla. Neurolmage 35: 539-552.

Smith SM, Jenkinson M, Woolrich MW, Beckmann CF, Behrens TEJ, Johansen-Berg H, Bannister PR, De Luca M, Drobnjak I, Flitney DE, Niazy RK, Saunders J, Vickers J, Zhang Y, De Stefano N, Brady JM, Matthews PM (2004) Advances in functional and structural MR image analysis and implementation as FSL. Neurolmage 23: S208-S219.

Sterr A, Muller MM, Elbert T, Rockstroh B, Pantev C, Taub E (1998) Perceptual correlates of changes in cortical representation of fingers in blind multifinger Braille readers. Journal of Neuroscience 18: 4417-4423.

Stringer EA, Chen LM, Friedman RM, Gatenby C, Gore JC (2011) Differentiation of somatosensory cortices by high-resolution fMRI at $7 \mathrm{~T}$. Neurolmage 54: 1012-1020.

Sur M, Nelson RJ, Kaas JH (1982) Representations of the body surface in cortical areas 3b and 1 of squirrel monkeys: Comparisons with other primates. The Journal of Comparative Neurology 211: 177-192.

Yacoub E, Shmuel A, Pfeuffer J, Van De Moortele P-F, Adriany G, Andersen P, Vaughan JT, Merkle $\mathrm{H}$, Ugurbil K, Hu X (2001) Imaging brain function in humans at 7 Tesla. Magnetic Resonance in Medicine 45: 588-594.

Yousry TA, Schmid UD, Alkadhi H, Schmidt D, Peraud A, Buettner A, Winkler P (1997) Localization of the motor hand area to a knob on the precentral gyrus. A new landmark. Brain 120: 141-157. 



\subsection{Feature-based attentional modulation of orientation perception in somatosensation}

In a human reaction-time experiment, we explored whether spatial and feature-based tactile cues could improve the subjects' perception. Using spatial attention, faster reaction times were observed to tactile targets at a cued location compared to those at an uncued location. Response time was also decreased for tactile targets with a cued compared to an uncued feature, not only at the cued location but also at a location where the cued and uncued target features were equally likely. Hence, the data provide the first documentation of a behavioral effect of feature-based attention for the modality of touch. 


\section{Feature-based attentional modulation of orientation perception in somatosensation}

Feature-based tactile attention

Meike A. Schweisfurth ${ }^{1,2}$, Steffen Katzner ${ }^{3}$, Renate Schweizer ${ }^{2}$, Stefan Treue ${ }^{1,4,5}$

${ }^{1}$ Cognitive Neuroscience Laboratory, German Primate Center, Goettingen, Germany, ${ }^{2}$ Biomedizinische NMR Forschungs $\mathrm{GmbH}$ am Max-Planck-Institut für biophysikalische Chemie, Germany, ${ }^{3}$ Werner Reichardt Centre for Integrative Neuroscience, University of Tuebingen, Germany, ${ }^{4}$ Faculty for Biology \& Psychology, Goettingen University, ${ }^{5}$ Bernstein Center for Computational Neuroscience, Goettingen

Correspondence should be addressed to: Meike Schweisfurth, Cognitive Neuroscience Laboratory, German Primate Center, Kellnerweg 4, 37077 Goettingen, Germany; Email: mschwei1@gwdg.de 

somatosensation

\section{Abstract}

In a reaction-time study of tactile orientation detection the effects of spatial attention and, for the first time, feature-based attention, were investigated.

Subjects had to give speeded responses to target orientations (vertical and horizontal) in a random stream of oblique tactile distractor orientations presented to their index and ring fingers. Before each block of trials, subjects received a tactile cue at one finger (cued location). The cue carried the information that the target would more likely appear at the cued location and was informative about which of the two possible target orientations would likely be presented (cued orientation) if the target should occur at the cued location. For targets presented at the uncued location, both target orientations were equally likely. Hence, subjects could benefit from attending to the cued location and only there to the cued orientation.

Faster reaction times were observed in location-matched trials, i.e. when targets appeared on the cued finger, representing a perceptual benefit of spatial attention. Additionally, reaction times were shorter in orientation-matched trials, both at the cued and at the uncued location, indicating a global enhancement of tactile sensation by feature-based attention.

Keywords: attention, behavior, feature-based, human, orientation, reaction time, spatial, tactile 


\section{Introduction}

Due to the brain's limited processing capacity, human perception does not provide a complete representation of the sensory input from the environment. Instead, our brain combines external bottom-up information with internal top-down influences to selectively improve the processing and perception of assumed to be relevant information. Voluntary attention is the major top-down influence for this selection process. It can lead to improved processing of attended locations, objects, and features. Decreased reaction times and higher accuracy rates are found for attended compared to unattended sensory signals (e.g. Posner et al., 1978). Even the perceived contrast and size of stimuli can be changed by attention (e.g. Carrasco et al., 2004; Anton-Erxleben et al., 2007). While attentional effects have been extensively studied in the visual domain, far less research has been devoted to somatosensory attention.

In touch, as in vision, the best-explored attentional phenomenon is spatial attention. Psychophysically, most research employed Posner (Posner, 1978) or Posner-like designs. In these, a target is to be detected at one out of several possible locations. The target presentation is preceded by a cue indicating the likely target location; targets presented at that location are called validly cued, in contrast to invalidly-cued targets that are presented at another location. Studies employing a Posner design with tactile discrimination task (different response buttons for different targets) often show faster reactions to validly-compared to invalidly-cued targets (Posner, 1978; Spence et al., 2000; Forster and Eimer, 2005; Chica et al., 2007; Van Ede et al., 2012) or higher accuracy for validly-cued targets (Sathian and Burton, 1991; Van Ede et al., 2012). Using a Posner design with a simple detection task (same response button for all targets), some studies reported spatial-orienting effects (Butter et al., 1989; Cohen et al., 2005) whereas others did not find them (Posner, 1978) or only partly (presence versus absence tasks of Sathian and Burton, 1991 and of Whang et al., 1991; Lloyd et al., 1999).

Whereas almost all attentional studies in touch have been focused on spatial attention, studies in the visual domain revealed that attention can not only be allocated to specific regions of visual space but also to specific features. Here, 'feature' refers to a particular property within a stimulus dimension. For example, 


\subsection{Feature-based attentional modulation of orientation perception in}

somatosensation

upwards motion is a feature within the stimulus dimension of motion direction and red is a feature within the stimulus dimension of color. Feature-based attention can be demonstrated on the level of single neurons in sensory cortex. If a monkey's attention is directed to the preferred feature (e.g. a color, a direction of motion) of a recorded neuron, even far outside its receptive field, the neuron's response will be increased (compared to a baseline where no feature is attended), whereas attention to the neuron's non-preferred feature results in a decreased response (Treue and Martinez-Trujillo, 1999). Feature-based attentional effects can be explained with the feature-similarity gain model, suggested by Treue and Martinez-Trujillo (1999). The global effect of visual feature-based attention has also been shown in human psychophysical studies (Rossi and Paradiso, 1995; Saenz et al., 2003), suggesting a higher accuracy for matching features, and human imaging studies (Saenz et al., 2002), reporting an increased $\mathrm{fMRI}$ response to an ignored stimulus of a given feature upon attention to a distant stimulus with the same feature compared to one with a different feature.

In the tactile domain, feature-based attention has only rarely been explored. Some studies explored the allocation of attention to one out of two stimulus dimensions (Burton et al., 1999, 2008; Hoechstetter et al., 2000; Sinclair et al., 2000). Behavioral data revealed decreased RTs for valid compared to neutral cueing of the target's dimension (Burton et al., 1999; Sinclair et al., 2000), whereas no cortical effects could be shown (Burton et al., 1999, 2008). However, feature-based attention as defined above, i.e. allocating attention to one out of several features within the same stimulus dimension, has only been investigated once in somatosensation, in an event-related potential (ERP) study by Forster and Eimer (2004). While reporting the first evidence for cortical correlates of global featurebased attention in touch, they did not explore whether these actually lead to improved behavior for attended versus unattended features.

In the present study, the behavioral effects of tactile spatial and feature-based attention were explored in a human reaction-time study, using orientation as the relevant stimulus dimension. The task of the subjects was to monitor a stream of tactile stimuli for the occurrence of one of two designated target orientations. A cue specified the likely location and orientation of the target stimulus. As the orientation cue was only informative for the cued location, subjects were asked to attend to the 
cued location and - only there - the cued orientation. We observed faster reaction times to orientation-matched compared to orientation-unmatched targets, both at the cued and the uncued location, indicating a global effect of tactile feature-based attention. 


\subsection{Feature-based attentional modulation of orientation perception in}

somatosensation

\section{Materials and Methods}

20 subjects (aged $24.8 \pm 3.3$ years (mean \pm standard deviation), 11 males and 9 females) participated in this study. All subjects were right-handed according to the Edinburgh Inventory (laterality index $0.9 \pm 0.1$, Oldfield, 1971). They gave their informed written consent before the experiment. The study was approved by the ethics committee of the Georg-Elias-Mueller-Institute for Psychology, Goettingen University.

Each subject participated in three sessions of two to three hours duration. The first session served as training, whereas the data recorded in the second and third sessions were used for analysis. Each session took place in a dimly illuminated and quiet testing room. Subjects sat on a comfortable chair, with their right foot placed on a foot pedal, such that it could be pressed by a small and effortless toe movement. The subjects' hands were placed on a table, centrally in front of the body. After stimulator positioning (described below), the hands were covered by a soundabsorbing box, that did not touch the hands but ensured that tactile stimulation patterns could not be differentiated by visual or acoustic information. Subjects were told to keep their eyes closed throughout a session.

\section{Stimuli}

Tactile stimulation of the right-hand index (D2) and ring (D4) finger was performed using a piezo-electric stimulation device (Piezostimulator, QuaeroSys, St. Johann, Germany) consisting of a control unit and two connected, custom-built stimulation modules. Each module was equipped with a 17-pin radial Braille display (Fig. 1A) consisting of one central pin surrounded by two 8-pin circles of radius $2.5 \mathrm{~mm}$ and $5.0 \mathrm{~mm}$, respectively. Each pin could be controlled individually.

By jointly elevating up to 5 pins arranged on a straight line through the central pin, 4 different orientations could be presented (Fig. 1B): Vertical (09, horizontal (perpendicular to vertical, 909 and the diagonal orientations in between $\left(45^{\circ}\right.$ and 1359. The stimulation displays were positioned bel ow the fingertips such that the vertical pin orientation was oriented in parallel to the proximal-to-distal fingertip axis and the central pin was located slightly distal to the fingertip vortex. Subjects were instructed to keep their right hand relaxed and pronated throughout the experiment. 
Figure 1. Stimulation. (A) Tactile radial stimulator. (B) Illustration of the 4 possible orientation patterns and their location relative to the proximal-to-distal fingertip axis. (C) Actually presented orientation realizations, exemplified with the vertical orientation.

A

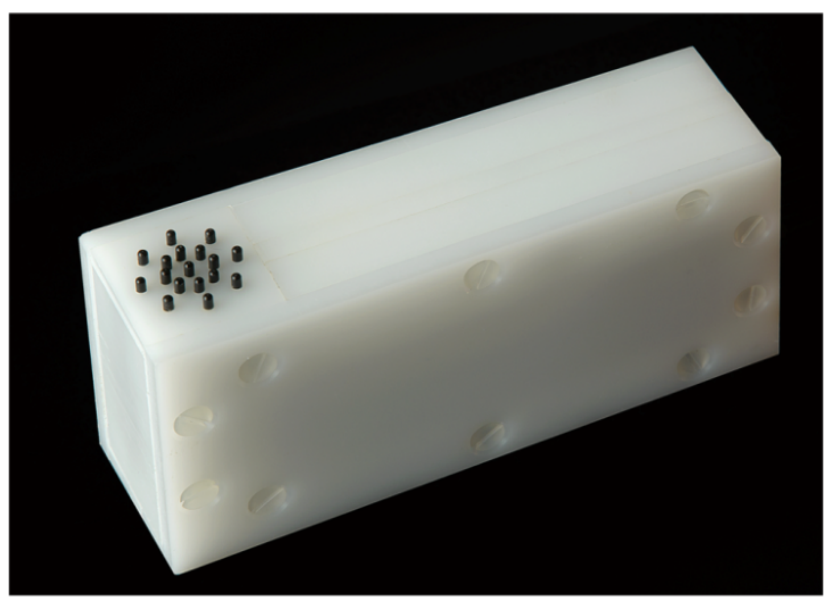

B

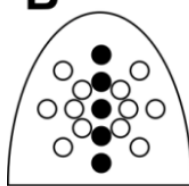

vertical
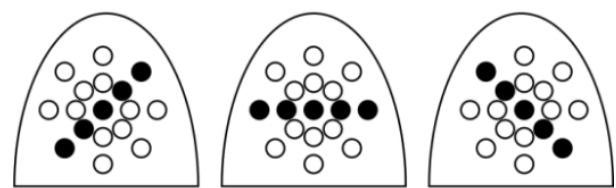

diagonal horizontal diagonal

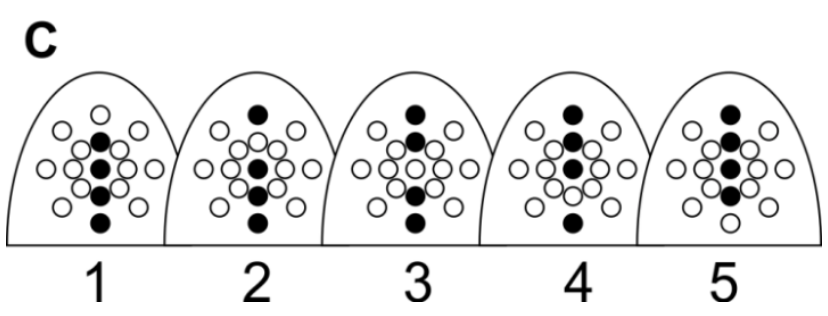

dent random sequences of distractor orientations (oblique) were presented simultaneously at the right-hand index (D2) and ring (D4) finger (Fig. 2A). The number of distractor presentations ( $3-15$, mean of 6 ) was gamma distributed $\sim \Gamma(7.5,0.8)$. Distractors were separated by $100 \mathrm{~ms}$. At some point, a vertical or horizontal orientation (the 'target') was presented at one of the locations. The subjects were instructed to give a speeded response by pressing the foot pedal. Upon response or $100 \mathrm{~ms}$ after target presentation, a mask stimulus was presented at both locations for $1 \mathrm{~s}$, generated by repeated presentation of every second pin. Distractor and target orientations were generated by simultaneously raising 4 pins randomly chosen from the 5 pins forming the given orientation (Fig. 1C). This 


\subsection{Feature-based attentional modulation of orientation perception in}

somatosensation

A

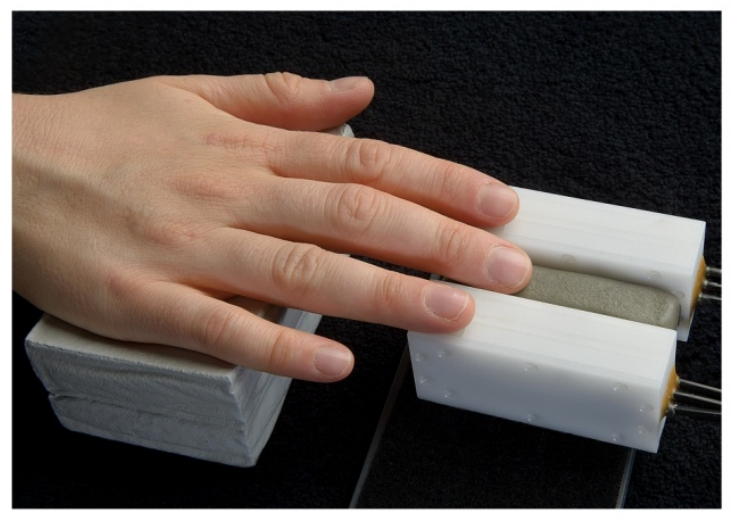

B

\section{D2-cued subjects}

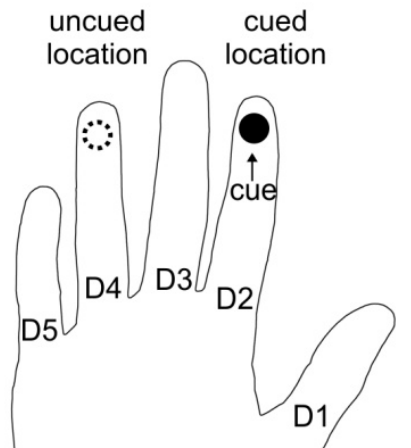

D4-cued subjects

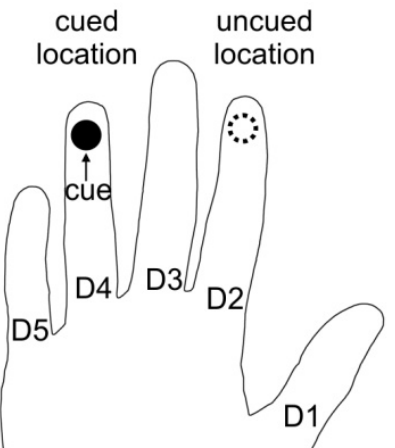

C
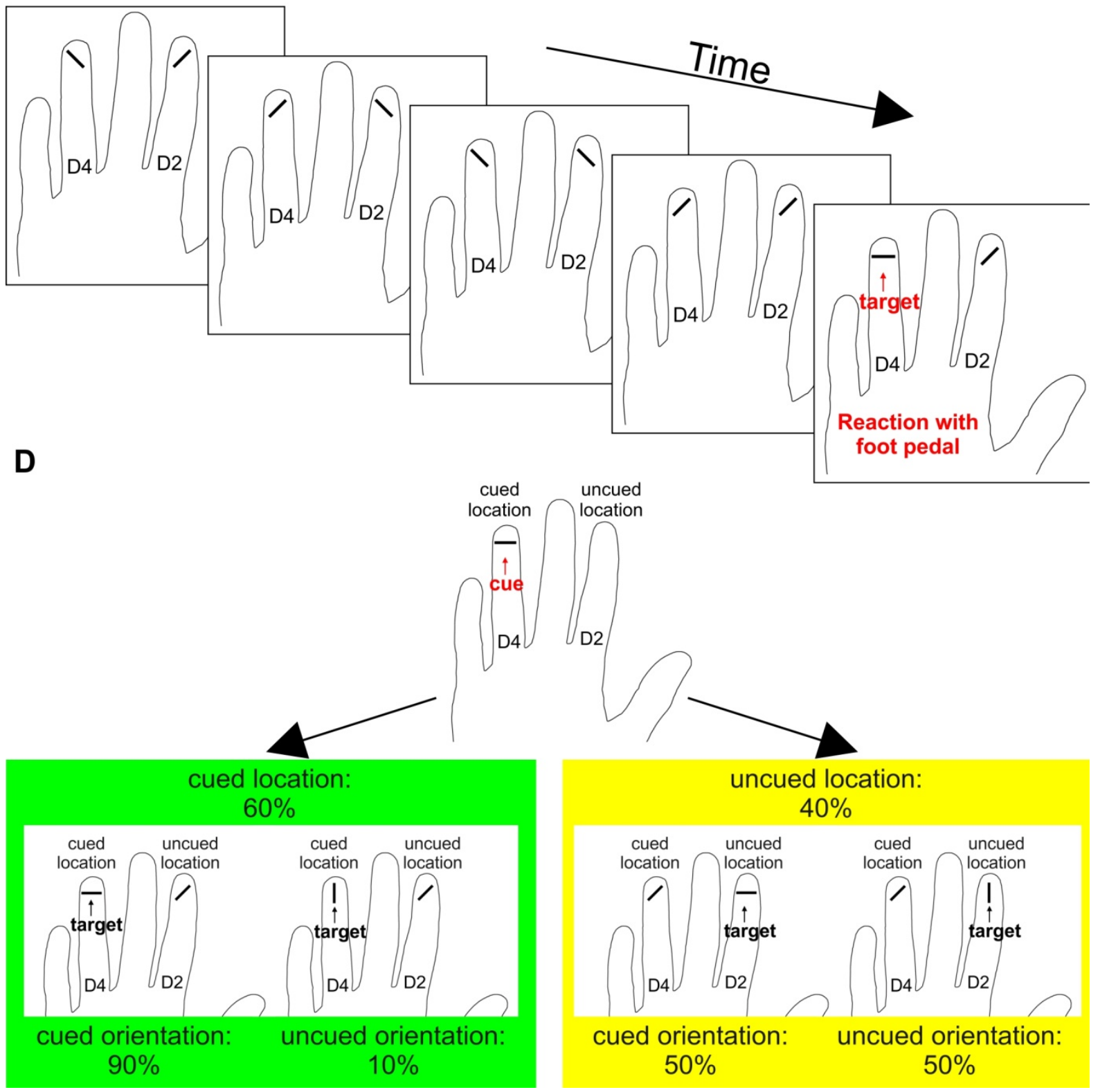
Figure 2. Positioning and paradigm. (A) procedure was applied to prevent Positioning. Positioning of the right-hand index (D2) and ring (D4) finger onto the two radial stimulators shown in Fig. 1. (B) Cue location. For both cuelocation groups (for D2-cued / D4-cued subjects in the left / right panel), the cued location (black circle with solid outline), to which all cues were presented, as well as the uncued location (white circle with dashed outline) are illustrated on a schematic hand.

(C) Example of trial timecourse. Within each trial, subjects had to attend to presentation of a random number of random diagonal orientations and react upon presentation of a vertical or horizontal orientation (target) at any of the two locations, which required a foot response of the subject. (D) At the beginning of each block, a cue with either horizontal (as shown in this panel, upper part) or vertical orientation was presented at one invariable location. Targets were more likely $(60 \%)$ to occur at that cued location (lower, green panel part) compared to the uncued location (40\%, lower, yellow panel part). At the cued location, the orientation of the cue was only informative, such that $90 \%$ of the targets presented there comprised with the same orientation as the cue (orientation-matched targets); at the uncued location, both target orientations appeared with same probability. Hence, in most of the trials (54\%), targets matched the location and orientation of the cue. In the remaining trials, targets matched the location, but not the orientation of the cue (6\% of all trials), or were presented at the uncued location with either matched or unmatched orientation ( $20 \%$ each).

subjects from concentrating on individual pins instead of abstract orientations.

In order to guide attention, each block started with a tactile cue (3 s duration), which was always presented at the same location. Half of the subjects received the cue at $\mathrm{D} 2$, the other half at D4 (Fig. 2B). The cue was location-informative, as the targets were displayed at the cued finger in $60 \%$ of the trials (Fig. 2D). Targets at this finger were therefore called location-matched, whereas targets at the other finger (only 40\%) were called location-unmatched. The cue was of either vertical or horizontal orientation (generated by elevation of all five pins) and orientation informative for location-matched targets, as these had the same orientation as the cue in $90 \%$ of the trials; for location-unmatched targets, the cue was non-informative, with vertical and horizontal targets being equally likely (Fig. 2D). A target was referred to as orientation-matched / -unmatched, if its orientation agreed / disagreed with the cue orientation. Subjects were instructed to make use of the information provided by the cue (i.e. location and orientation).

After each block, subjects could choose whether to go on or take a break in order to be able to maintain their level of concentration and tactile sensitivity. They were instructed to take at least one break within 100 trials.

\section{Analysis}

Only reaction times (RTs) between $250 \mathrm{~ms}$ and $1350 \mathrm{~ms}$ after target onset were used for analysis, as shorter RTs likely were responses to the previous 


\subsection{Feature-based attentional modulation of orientation perception in}

somatosensation

distractor and longer ones responses to the mask. RTs of each subject were sorted into the different combinations of target location and target orientation. For each subject and for each target location separately, RTs from each session were normalized to the subject's overall mean and standard deviation and pooled across the two recording sessions in the following way. First, the population mean and standard deviation (SD) were calculated for each target location, both separately for each session and jointly for both sessions (resulting in grand mean and grand SD). Then, separately for each session, the RTs were transformed into z-scores (by subtraction of the session mean followed by division with the session SD). Finally, these z-scores were transformed into normalized RTs by multiplication with the grand SD followed by addition of the grand mean. These normalized RTs could then be pooled across the two sessions of a subject and were used for further analysis.

Statistical analysis of RTs between attentional conditions was performed in SPSS (version 16.0). A 4-way mixed analysis of variance (ANOVA) was conducted with the across-subjects factor cue-location group (D2-cued / D4-cued subjects) and the three within-subject, target-property factors location validity (location matched / unmatched), target orientation (vertical / horizontal), and orientation validity (orientation matched/unmatched). Significant two-way interactions were broken down by simple-effects analysis, i.e. by pooling RTs across all but the two interacting factors and then calculating paired t-tests between two levels of one factor, separately for the two levels of the other factor. Cohen's d (Cohen, 1988; Erdfelder et al., 1996) was used as a measure of effect size. 


\section{Results}

The focus of our study was to explore the effect of spatial and feature-based attention on behavioral performance. After cueing one of two locations (either index or ring finger) and one of two orientations (either vertical or horizontal, cue only informative for target presentations at the cued location), the subjects' task in each trial was to monitor two parallel sequences of oblique tactile distractors presented to the two fingers and react as soon as a target orientation (either vertical or horizontal) was presented at one of the fingers (Fig. 2). Subjects were instructed to attend to the cued location and orientation throughout a trial, as targets were more likely to appear at the cued location and with the cued feature (orientation). Comparing reaction times (RTs) to different combinations of target location and orientation, we assessed the effects of tactile spatial and feature-based attention. The analysis showed reduced RTs when attention allocation matched the target, i.e. subjects reacted faster to location-matched compared to location-unmatched and to orientationmatched compared to orientation-unmatched targets.

The average task accuracy across the 20 subjects was $84.3 \pm 5.6 \%$, such that out of the 800 trials recorded per subject, on average $674 \pm 45$ could be used for further analysis. $7.6 \pm 3.0 \%$ (mean \pm standard deviation) of the trials were excluded as early responses. $8.1 \pm 4.6 \%$ of the trials were excluded as late responses (or because no response was given at all).

The average RTs as a function of the 4 factors of interest (cue-location group, location validity, target orientation, and orientation validity) are listed in Table 1. In Fig. 3 the average RTs are plotted, grouped by the validity of the location cue (location-matched vs. location-unmatched) to visualize spatial attention effects. Within each validity condition the data are grouped by the orientation of the target (vertical vs. horizontal) to visualize differences in overall orientation sensitivity. For any given target orientation the RTs are separated by the validity of the orientation cue (orientation-matched vs. orientation-unmatched) to visualize effects of featurebased attention. The statistical significance of RT differences between conditions was evaluated with an overall 4-way mixed ANOVA. The various effects are described in the following subsections. 


\subsection{Feature-based attentional modulation of orientation perception in somatosensation}

Table 1. Reaction times grouped with respect to target properties. Separately for each cuelocation goup, the reaction times (mean \pm repeated-measures standard deviation) in response to targets are listed for each set of target properties, i.e. for each combination of location validity, target orientation, and orientation validity.

\begin{tabular}{|c|c|c|c|c|c|c|c|c|c|c|}
\hline $\begin{array}{l}\text { Location } \\
\text { validity }\end{array}$ & + & $\begin{array}{l}\text { Target } \\
\text { orientation }\end{array}$ & + & $\begin{array}{l}\text { Orientation } \\
\text { validity }\end{array}$ & D2-cue & su & $\begin{array}{l}\text { bjects } \\
D[\mathrm{~ms}]\end{array}$ & $\begin{array}{r}\text { D4-cu } \\
\mathrm{N}\end{array}$ & d st & $\begin{array}{l}\text { bjects } \\
\text { D [ms] }\end{array}$ \\
\hline matched & + & vertical & + & matched & 555 & \pm & 70 & 640 & \pm & 58 \\
\hline matched & + & vertical & + & unmatched & 657 & \pm & 39 & 733 & \pm & 63 \\
\hline matched & + & horizontal & + & matched & 609 & \pm & 60 & 728 & \pm & 45 \\
\hline matched & + & horizontal & + & unmatched & 714 & \pm & 65 & 826 & \pm & 56 \\
\hline unmatched & + & vertical & + & matched & 728 & \pm & 73 & 773 & \pm & 48 \\
\hline unmatched & + & vertical & + & unmatched & 742 & \pm & 57 & 798 & \pm & 47 \\
\hline unmatched & + & horizontal & + & matched & 812 & \pm & 60 & 846 & \pm & 63 \\
\hline unmatched & + & horizontal & + & unmatched & 819 & \pm & 40 & 879 & \pm & 70 \\
\hline
\end{tabular}

Figure 3. Reaction times grouped with respect to target properties. Separately for each cuelocation group (for D2-cued / D4-cued subjects depicted by filled circles / open squares), the mean reaction times (mean and repeated-measures 95\% confidence intervals) are plotted. Responses to location-matched / location-unmatched targets are shown in the left /right half of the plot. Further on, these halfs are divided into vertical (left) and horizontal (right) targets. The abscissa further sorts between orientation-matched (left) and orientation-unmatched targets (right).

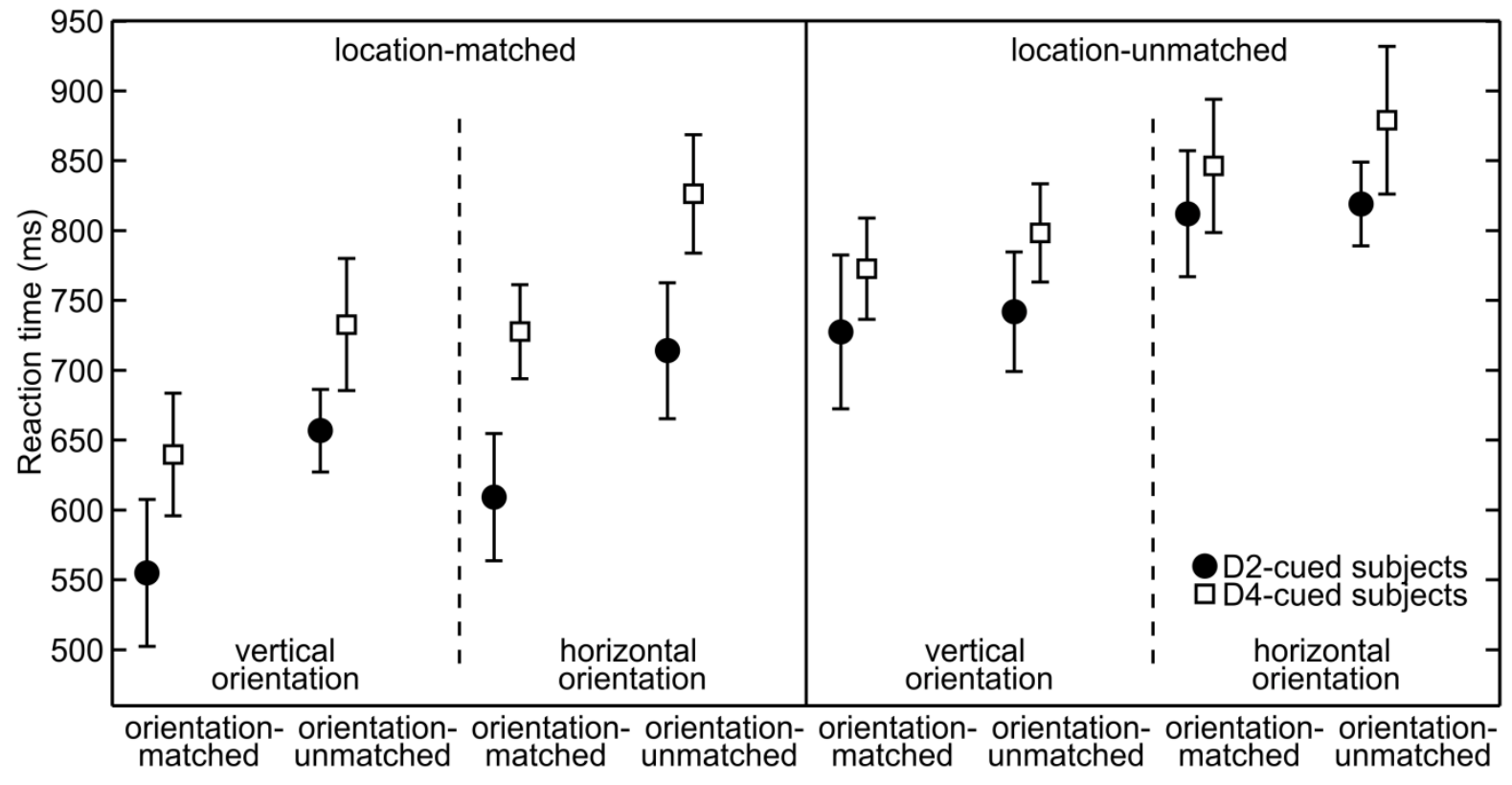

\section{Feature-based attention}

RTs to targets with the cued orientation were on average $60 \mathrm{~ms}$ shorter than orientation-unmatched targets. This is apparent in Fig. 3, as under otherwise identical conditions (neighboring same-marker data points without line separation) RTs to orientation-matched targets (left errorbar) are lower than those to orientationunmatched targets (right errorbar). This observation was statistically confirmed by a significant main effect of orientation validity in the RM ANOVA (mean 
Figure 4. Interaction between location validity and orientation validity. Reaction times (mean and repeated-measures $95 \%$ confidence intervals, pooled across cue-location groups and target orientations) are shown for each combination of location validity and orientation validity. The values for targets with different orientation validities but same location validity are connected (green markers for location-matched, yellow for locationunmatched targets). Comparing them statistically ( $p$-value stated), both at the cued and at the uncued location a decrease in reaction time from orientation-unmatched to orientation-matched targets is found, with the effect being more pronounced for location-matched targets.

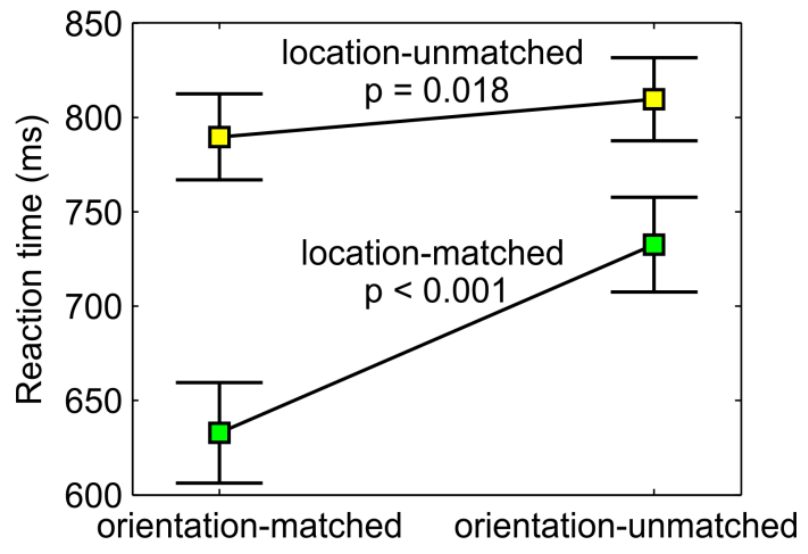

the stronger RT increase at the cued location was revealed by a significant interaction between the factors location validity and orientation validity $(F(1,18)=48.1, p<0.001)$. Follow-up simple-effects analysis confirmed that RTs to the cued orientation were significantly faster both at the cued and at the uncued location. While the effect was large was for location-matched targets $(M=-99 \mathrm{~ms}$, $S D=66 \mathrm{~ms}, t(19)=-6.79, p<0.001$, effect size $d=1.5)$, it was of medium effect size for location-unmatched targets $(M=-20 \mathrm{~ms}, S D=23 \mathrm{~ms}, t(19)=-2.59$, $p=0.018, d=0.6)$. Hence, feature-based allocation of attention not only had an influence at the location where the feature-based cue was informative, but also at the location without any previous feature-based information, pointing to a global effect of feature-based attention.

\section{Spatial attention}

At the cued location, subjects reacted on average $117 \mathrm{~ms}$ faster than at the uncued location. This effect is reflected in Fig. 3, where RTs to otherwise same 

somatosensation

conditions are lower in the left compared to the right half of the plot. Statistically, it was confirmed by a significant main effect of location validity $(M=-117 \mathrm{~ms}, \mathrm{SD}=$ $89 \mathrm{~ms}, \mathrm{~F}(1,18)=35.6, \mathrm{p}<0.001)$. Hence, spatial allocation of attention resulted in decreased RTs at the cued location.

\section{Orientation anisotropy}

Responses to vertical targets were on average $76 \mathrm{~ms}$ faster than responses to horizontal targets. This can be estimated from Fig. 3, as RTs to the vertical orientation (data points to the left of dashed lines) are lower than those to the horizontal orientation (data points to the right of dashed lines) under otherwise identitical conditions. The effect was statistically confirmed by a significant main effect of target orientation $(M=-76 \mathrm{~ms}, S D=47 \mathrm{~ms}, F(1,18)=51.9, p<0.001)$. The RT difference between targets with vertical and horizontal orientation pointed to an anisotropy in orientation processing or perception.

\section{Further results}

Subjects for whom the index finger formed the cued location tended to respond faster than D4-cued subjects. That trend is reflected in Fig. 3, where the black marker tends to be lower than its adjacent white marker. However, the trend did not reach statistical significance, as seen by the main effect of cue-location group $(\mathrm{M}=-73 \mathrm{~ms}, \mathrm{~F}(1,18)=3.5, \mathrm{p}=0.079)$.

All interactions between within-subject factors were far from significant $(F(1,18)<1, p>0.7)$, except for the already discussed interaction between location validity and orientation validity. Also, all interactions of the across-subject factor cuelocation group with one, two, or three of the within-subject factors proved insignificant $(F(1,18)<1.5, p>0.2)$. 


\section{Discussion}

To explore the existence of spatial and feature-based attention in the somatosensory system, we studied their influence onto human reaction times (RTs). Subjects had to report the presentation of target orientations (vertical and horizontal) to their index and ring fingers, ignoring oblique distractor orientations. Feature-based cues were informative for only one of the stimulated locations. Nevertheless, decreased RTs to the cued orientation were found at both target locations, demonstrating a behavioral effect of global tactile feature-based attention.

\section{Feature-based attention}

Using an elaborate psychophysical design, we could show perceptual benefits when the subjects' attention was directed to behaviorally-relevant tactile features. Responses were faster for orientation-matched targets at the location for which the feature-based cue was orientation-informative (cued location).

In many other psychophysical studies, Posner-like RT paradigms have been used and whenever RTs decreased for validly- compared to invalidly-cued features or locations, this has been described as attentional effect. However, Duncan (1980) and Sperling (1984) have shown that improved perceptual performance is not necessarily evidence for more efficient cortical processing (a consequence of attentional selection). Decreased RTs can also result from lowering the amount of information required for triggering a response, i.e. by decreasing the level of required certainty. As a target at the cued location was most likely to be of the cued orientation (90\%), the effect of faster RTs for valid targets at the cued location might, at least partly, result from a higher expectancy of matched compared to unmatched targets (see also LATER analysis in Supplementary Material).

Crucially, however, decreased RTs were not only observed at the cued location but also at the uncued location, pointing to a global effect of tactile featurebased attention. At the uncued location, the effect should not be due to different degrees of certainty between valid and invalid targets, as both conditions appeared equally often.

The global effect of feature-based attention we observed is similar to the one reported by psychophysical studies in vision (Rossi and Paradiso, 1995; Alais and 


\subsection{Feature-based attentional modulation of orientation perception in}

somatosensation

Blake, 1999; Saenz et al., 2003; Arman et al., 2006) and represents the first report of behavioral effects of feature-based attention in touch. Our findings are well complemented by the only other study on tactile feature-based attention (Forster and Eimer, 2004), which reported cortical evidence for global effects. In their study, event-related potentials (ERPs) were recorded upon delivery of tactile stimuli presented to the right or left hand. Stimuli were of low or high frequency (first experiment) or of low or high intensity (second experiment). Subjects had to attend simultaneously to one of the stimulus locations and to one of the non-spatial features. ERP analysis revealed effects of feature-based attention (enhanced negativities to the attended frequency or intensity), independently of the current focus of spatial attention, suggesting a global effect of feature-based attention. Perceptual effects were not assessed. Further imaging studies will be necessary to identify the cortical regions in which tactile feature-based attention operates.

\section{Spatial attention}

Our data show that responses to targets at the cued location are much faster compared to those at the uncued location, in line with several previous studies (Posner, 1978; Spence et al., 2000; Chica et al., 2007) reporting a behavioral RT effect of spatial cueing in touch. However, it is unclear whether these effects are due to faster processing resulting from allocation of spatial attention or due to lowering the amount of information required for triggering a response resulting from the higher probability for a location-matched target (see LATER analysis in Supplementary Material).

\section{Finger anisotropy}

Across subjects we observed a trend for lower RTs to targets presented to D2 (729 $\pm 30 \mathrm{~ms}$, mean \pm standard error) compared to targets at D4 (753 $\pm 23 \mathrm{~ms})$. This finding is in agreement with a study by Vega-Bermudez and coworkers (2001) who reported that tactile acuity in a letter-recognition and in a grating-orientation discrimination task progressively declined from D2 to D4, suggesting anisotropic sensitivities between the fingers, possibly because of the more important role of the index finger (compared to the ring finger) in everyday hand-use. 


\section{Orientation anisotropy}

In the literature, there is an ongoing debate about anisotropic processing and perception of tactile orientations. Lechelt (1988) reported better detection of deviations from horizontal compared to vertical orientations. Similarly, Bensmaia and coworkers (2008b) observed a lower angular deviation-detection threshold for the horizontal compared to the vertical orientation and a trend for better performance for horizontal compared to vertical bars, both in an orientation-discrimination and in a convergence-detection task. For studies involving gratings, even the definition of vertical versus horizontal orientation varies between studies (Gibson and Craig, 2005). Here, a grating is defined as vertical if it is build up from proximal-to-distal bars in parallel to the finger axis. Essock and colleagues (1997) reported that sensitivity to detection of gratings (versus blanks) was best for vertical gratings and worst for horizontal ones; the results could not be replicated by a similar study by Craig (1999), whereas Gibson and Craig (2005) found similar results for the finger location stimulated in our design (defined as fingerpad in their study). In a gapdetection and in a grating orientation (GR/OR) task, however, these authors could not find anisotropy between the vertical and the horizontal orientation. In a monkey study (DiCarlo and Johnson, 2000) exploring the primary somatosensory cortex, 'vertical' was reported less often as a neuron's preferred orientation than other orientations (not statistically tested), whereas a more recent study (Bensmaia et al., 2008a) did not find any orientation to be overrepresented in SI.

Our results clearly show anisotropy between target orientations, as vertical targets led to significantly faster responses than horizontal targets. Without being questioned, seven out of the 20 subjects stated that the detection of vertical targets was easier for them, while none claimed the contrary. Interpreting the results in the light of the existing literature, one could argue that faster responses to vertical targets resulted from worse detection of deviations from a vertical compared to those from a horizontal standard orientation (Lechelt, 1988; Bensmaia et al., 2008b). Oblique-stimulus presentation before target appearance might further alter the subjects' perception of vertical and horizontal orientation. As even large deviations from the vertical orientation might still be categorized as 'vertical', vertical targets might have appeared clearer and hence were faster detectable. 
2.3 Feature-based attentional modulation of orientation perception in somatosensation

\section{Conclusion}

In conclusion, this study not only confirms effects of tactile spatial attention but is the first to report behavioral effects of tactile feature-based attention, acting not only on a local but on a global scale, similar as observed in vision. The presence of feature-based effects at the uncued location strongly supported that behavior was not (only) altered due to cue-related modification in expectancies but due to altered cortical information processing due to feature-based attention. Further cortical studies are needed to precisely locate from which brain areas the reported behavioral effects of feature-based attention originate. 


\section{Acknowledgements}

The current work was supported by the International Max Planck Research School MSc/PhD/MD-PhD Neuroscience Program (to M.A.S.) of the Göttingen Graduate School of Neurosciences and Molecular Biosciences (GGNB) in Göttingen, Germany, and the graduate school Neurosenses (to M.A.S.) in Göttingen and Oldenburg, Germany. 


\section{Supplementary material (LATER analysis)}

In the main part of this paper we have shown that subjects react faster to targets with cued compared to uncued location and feature. As already touched upon in the discussion, these decreased RTs can principally be due to a Bayesianinduced decrease in the information amount required to respond or due to an attention-induced improvement of cortical information processing. Here, simple riseto-threshold models (Carpenter and Williams, 1995; Reddi et al., 2003) are used to explore which of the two mechanisms plays the more dominant role for the effects of spatial and feature based attention observed in the main part of this paper.

\section{Methods}

LATER (linear rise to threshold with ergodic rate) models were applied to the RTs of individual subjects (Carpenter, 1981; Carpenter and Williams, 1995; Reddi et al., 2003). According to LATER (Suppl. Fig. S1A), upon stimulus onset (bottom of panel) a decision signal (S, middle of panel) is triggered, which increases linearly from a start level $\left(S_{0}\right)$ to a threshold $\left(S_{T}\right)$. Upon reaching this threshold, a behavioral response is initiated (top of panel). The rate of information supply (slope, $r$ ) with which the decision signal increases is Gaussian distributed across trials. According to LATER, RTs are proportional to the quotient of distance to threshold $\left(\mathrm{S}_{\mathrm{T}}-\mathrm{S}_{\mathrm{O}}\right)$ and slope, $\mathrm{RT} \sim\left(\mathrm{S}_{\mathrm{T}}-\mathrm{S}_{0}\right) / \mathrm{r}$ (Reddi et al., 2003), and hence follow a recinormal distribution (i.e. their reciprocal is normally distributed), which can be verified in a reciprobit plot (Suppl. Fig. S1B).

LATER models with two processes rising towards threshold were chosen, one with positive-mean and one with zero-mean rate (Suppl. Fig. S1B). In the standard formulation of the LATER model, a single process is assumed to rise towards the threshold. Often, however, a number of trials (5 - 10\%) is observed, in which RTs are too fast to be explained by a single process (mainly described for saccadic responses (Carpenter and Williams, 1995; Carpenter and McDonald, 2007) but also for foot responses (Fort et al., 2010)). To describe these faster RTs, a second, zeromean process can be included (Suppl. Fig. S1B). This has also been done in our analysis here, because initial visual reciprobit-plot analysis revealed a substantial number of relatively fast RTs (Carpenter and Williams, 1995). As between-factor 
Supplementary Figure 1. The LATER model. Figure with permission adapted from Katzner et al. (2012). (A) The LATER model, adapted from Reddi et al. (2003). (B) Demonstration of a reciprobit plot in which the abscissa represents the reciprocal of reaction time with reversed direction, terminating at infinity. The ordinate is a probit scale, which is the inverse of the normal cumulative distribution function. These transformations turn a recinormal distribution into a straight line; the median of the RT distribution is given by the RT corresponding to the $z$ score of 0 . As a two-process LATER model was used for analysis, distributions with positive mean-rate (solid line) and zero mean-rate (dashed line) are shown. (C) Swivel variant of the LATER model, taking into account only adjustment of the distance to threshold for explanation of RT-distribution changes between conditions. Such changes lead to a swivel around the infinite time intercept within the reciprobit plot. (D) Shift variant of the LATER model, taking into account only changes in mean information supply (mean slope Mr) for explanation of RTdistribution changes between conditions. Such changes lead to a horizontal shift within the reciprobit plot.

interactions were non-significant (between all factors except for the interaction between location validity and orientation validity, see ANOVA analysis in main part), targets were pooled across cue-location groups and target orientations (and for spatial analysis also across orientation validities) for LATER analysis.

Two variants of this two-process LATER model were fitted with maximum-likelihood estimation, each modelling the RT distributions obtained in valid and invalid conditions, separately for spatial and feature-based analyses. In the swivel variant, only the distance to threshold $\left(\mathrm{S}_{\mathrm{T}}-\mathrm{S}_{0}\right)$ was allowed to vary, in the shift variant only the mean slope (Mr). Hence, both models consisted of 5 parameters: mean (Mr, 1 value for swivel, 2 for shift) and standard deviation (SDr) of the slope, distance to threshold $\left(\mathrm{S}_{\mathrm{T}}-\mathrm{S}_{0}, 2\right.$ values for swivel, 1 for shift), and standard deviation of the second, zero-mean process.

\section{A LATER model}

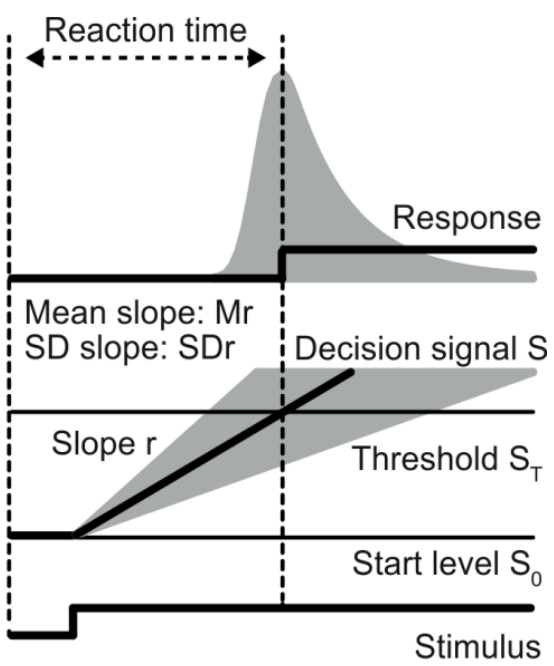

\section{B Reciprobit plot}

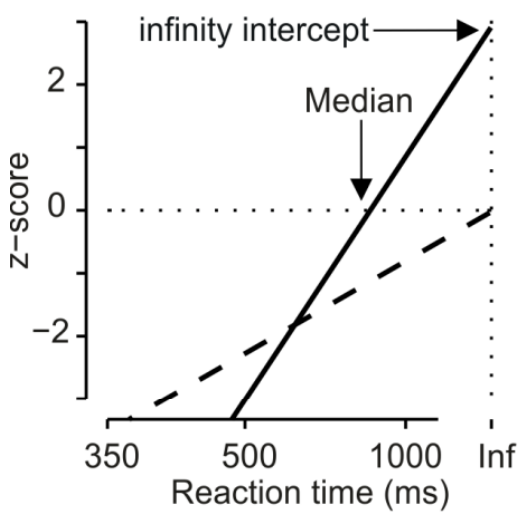

C Swivel variant

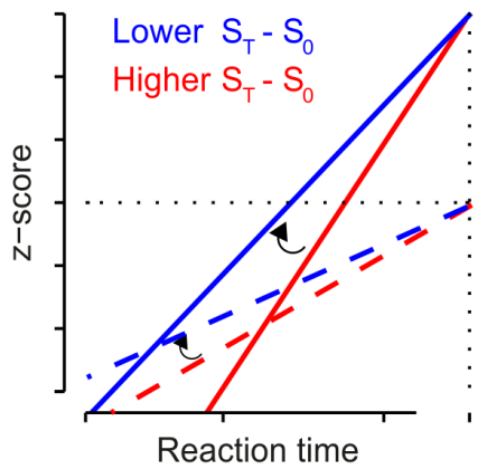

\section{Shift variant}

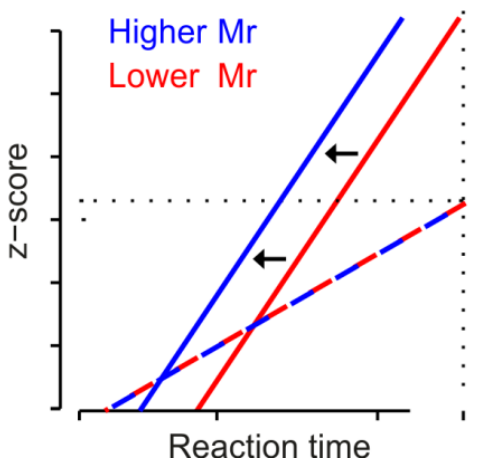




\subsection{Feature-based attentional modulation of orientation perception in}

somatosensation

For each subject, conformity to the LATER models was tested by one-sample Kolmogorov-Smirnov tests, which assessed whether the RT data did not differ from the model predictions. Taking into account only those subjects whose RTs were conform with the swivel or shift variant, the within-subject difference in log likelihood between swivel and shift variant was explored (paired t-test, $p<0.025$ for the feature-based and $p<0.05$ for the spatial analysis).

As the alternative models were of equal complexity (same number of degrees of freedom), potential model superiority was determined by this difference in log likelihood (Reddi et al., 2003; Katzner et al., 2012).

\section{Results}

For assessing whether RT differences rather resulted from changes in distance to threshold or in rate of information supply, the LATER model (Carpenter and Williams, 1995; Reddi et al., 2003) was applied to the RT data. Given this model, we assessed how attentional manipulations affected the distribution of RTs. The higher likelihood of an matched target could result in a decreased distance to threshold, in a higher mean rate of information supply, or in both, all leading to decreased RTs (Suppl. Fig. S1A). The former two alternatives can be modelled and have a characteristic signature on the main process in the reciprobit plot (Suppl. Fig. S1, solid lines): Reducing the distance to threshold would result in a left-swivel around the $z$-value corresponding to $\mathrm{RT}=\infty$ (swivel variant, panel $\mathrm{C}$ ). In contrast, a change in information supply would be reflected by a left-shift of the straight line (shift variant, panel D). The second process would be unaffected by changes in rate of information supply (panel D); changes in distance to threshold, however, would lead to swiveling of it (panel C). The two models were applied to explore the mechanism underlying feature-based and spatial effects.

Feature-based LATER analysis. Feature-based LATER analysis revealed model superiority (log likelihood difference of 1.57 ) for the swivel variant at the cued location, whereas no model outperformed the other at the uncued location. Out of the 20 subjects, for location-matched targets 17 subjects (6 of these making use of the zero-mean process) and for location-unmatched targets 20 subjects (15 of these making use of the zero-mean process) presented with RTs conform to the swivel 
variant (17 / 20 subjects for locationmatched / location-unmatched targets) or the shift variant (15/20 subjects for location-matched/ location-unmatched targets) of the LATER model and could be used for further analysis.

Data from an exemplary subject is shown in Suppl. Fig. S2 (panel A1 and B1 for the cued and uncued location, respectively), exemplary fitted with the swivel variant. For location-matched targets, statistics revealed strong model superiority for the swivel model (Suppl. Fig. S2, panel $A 2, M=1.57$, $\mathrm{SD}=1.56, \mathrm{t}(16)=4.14, \mathrm{p}<0.001$, $d=1$, i.e. large effect), whereas no difference was found for locationunmatched targets (Suppl. Fig. S2, panel $B 2, \quad M=0.11, \quad S D=2.75$, $t(19)=0.18, p=0.862)$. Hence, at the cued location, faster responses to the cued orientation mainly resulted from adjustments in the distance to threshold, whereas the observed RT difference at the uncued location was equally well explained by both models.

\section{Spatial LATER analysis.}

Spatial LATER analysis did not reveal any model superiority between swivel
Supplementary Figure 2. LATER analysis. The results for the LATER analysis are shown for one exemplary subjects (left column) and the group analysis (right column) for the feature-based analysis for location-matched (upper row) and locationunmatched (middle row) targets as well as for the spatial analysis (lower row). Left column (panel A1, B1, C1): For D2-cued Subject 3, reciprobit plots show the respective two measured reaction-time (RT) distributions (drawn as dots). Target validity (in orientation for the feature-based analysis and in location for the spatial analysis) is coded by color (blue for matched, red for unmatched). Solid lines represent maximum-likelihood estimates of the twoprocess LATER model, exemplary under the swivel constraint. The express component is represented by a zero-mean process, whereas the main, positivemean component fits most of the data. Right column (panel A2, B2, C2): Group log likelihood analysis between swivel and shift variant. Taking into account each dataset conform with the two-process swivel or shift model, the difference in log likelihood between swivel and shift variant was assessed. Mean and repeated-measures $95 \%$ confidence intervals are shown, statistical p-values are stated. In the featurebased analysis, for location-matched targets the swivel variant proved statistically superior to the shift variant (indicated by asterisk).
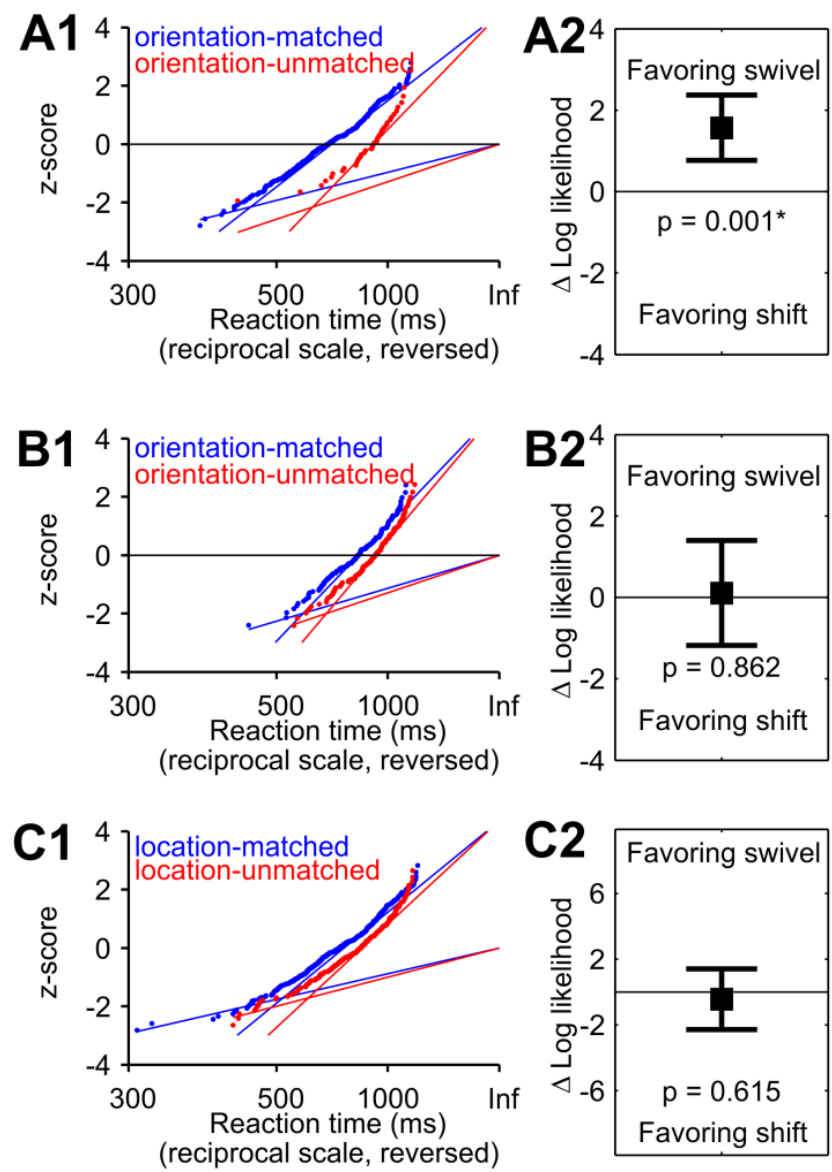


\subsection{Feature-based attentional modulation of orientation perception in}

somatosensation

and shift variant. Data from an exemplary subject is shown in Suppl. Fig. S2 (panel C1), exemplary fitted with the swivel variant. Out of the 20 subjects, 15 subjects (11 of these making use of the zero-mean process) presented with RTs conform to the swivel (13 subjects) or the shift (13 subjects) variant of the LATER model and could be used for further analysis. Statistically (Suppl. Fig. S2, panel C2), no model superiority could be determined between the swivel and the shift variant (mean difference in log likelihood $(M)=0.44$, standard deviation $(S D)=3.33, t(14)=-0.51$, $p=0.615)$. Hence, according to LATER, differences in RTs were equally well explained by adjustments in distance to threshold and adjustments in information supply.

\section{Discussion}

To differentiate between RT differences resulting from changes in distance to threshold or from changes in rate of information supply, the LATER model deals with an ideal Bayesian decision-making process which accumulates evidence until a certain critical amount of evidence for the target is acquired. In the LATER framework, the mean of an RT distribution is decreased upon several changes: One possibility is faster cortical processing by increasing the rate of information supply (shift model, Reddi and Carpenter, 2003), for example induced by attention. A nonattentional cause is an increased prior probability of the cued target feature or an increased response urgency for it, both decreasing the information amount required to trigger a response (swivel model, Carpenter and Williams, 1995; Reddi and Carpenter, 2000). Using the LATER model (Suppl. Fig. S1, Carpenter and Williams, 1995; Reddi and Carpenter, 2003), we tried to explore the causes for the $\mathrm{RT}$ decrease to targets with the cued compared to the uncued orientation and to targets at the cued compared to the uncued location.

At the cued location, the strong feature-based effect of decreased RTs to the cued compared to the uncued orientation turned out to be mainly due to a Bayesianinduced effect. This model superiority of the swivel compared to the shift model was expected due to the much higher prior probability of orientation-matched $(90 \%$ of the targets presented at the cued location) compared to orientation-unmatched (10\%) targets. For ideal observers, this should (according to LATER) indeed increase the start level of the decision-making process and thereby decrease the information 
amount required to respond. It would have been interesting to explore whether early responses primarily resulted from assumed orientation-matched targets, indicating a lower accuracy for these and hence a speed-accuracy tradeoff. However, the design did not allow for classification of early responses. Although the rate-adapting shift variant of the LATER model performed worse, a substantial number of RTdistributions (only two less than for the other model) were still found to be conform with it. Hence, though the RT decrease might primarily be due to Bayesian preparation, it was at least partly explainable by a higher processing-rate, indicating feature-based attention. This assumption was further supported by feature-based effects observed at the uncued location, which most probably spread over from feature-based attention directed to the cued orientation at the cued location.

At the uncued location, the slight RT decrease for orientation-matched compared to orientation-unmatched targets could not be ascribed to just one of the two possible modulatory mechanisms and hence assumed higher prior probability or feature-based attention might both have contributed to the effect. This was concluded because all subjects were conform to both LATER variants and because no model turned out to be superior to the other, which might be due to the small difference between the observed distributions for orientation-matched and orientation-unmatched targets (see Suppl. Fig. S2, panel B, for an example). However, a decrease in required information amount would be unexpected, as there was no higher prior probability for orientation-matched compared to unmatched targets at the uncued location. It might have been hard, however, for the subject to restrict the assumption of increased prior probability to the intended (i.e. cued) location. More probable is that the RT decrease was due to feature-based attention. The latter was probably directed to the cued location, in order to better process the cued orientation there. Similar as in visual psychophysical experiments (Rossi et al., 1995), feature-based attention must then have spread over to the uncued location.

The spatial LATER analysis revealed that the much faster responses observed at the cued compared to the uncued location were explainable by both attentional and Bayesian effects, as they could be equally well explained by the two respective model variants. Hence, the RT reduction at the cued location was indeed well explainable by spatial attention, as assumed by most previous studies (Posner, 1978; Spence et al., 2000; Chica et al., 2007). This theory is further corroborated by 
2.3 Feature-based attentional modulation of orientation perception in somatosensation

observations of improved accuracy (Sathian and Burton, 1991; Van Ede et al., 2012) and modulated cortical processing (Forster and Eimer, 2005) upon spatial allocation of attention. However, in the present study faster RTs were equally well explained by the slightly higher prior probability for the cued location, possibly leading to a decreased information amount required to respond. Hence, both mechanisms may play a role in spatial allocation of attention. 


\section{References}

Alais D, Blake R (1999) Neural strength of visual attention gauged by motion adaptation. Nature Neuroscience 2: 1015-1018.

Anton-Erxleben K, Henrich C, Treue S (2007) Attention changes perceived size of moving visual patterns. Journal of Vision 7: 1-9.

Arman AC, Ciaramitaro VM, Boynton GM (2006) Effects of feature-based attention on the motion aftereffect at remote locations. Vision Research 46: 2968-2976.

Bensmaia SJ, Denchev PV, Dammann III JF, Craig JC, Hsiao SS (2008a) The representation of stimulus orientation in the early stages of somatosensory processing. Journal of Neuroscience 28: 776-786.

Bensmaia SJ, Hsiao SS, Denchev PV, Killebrew JH, Craig JC (2008b) The tactile perception of stimulus orientation. Somatosensory and Motor Research 25: 49-59.

Bertelson P (1966) Central intermittency twenty years later. The Quarterly Journal of Experimental Psychology 18: 153-163.

Burton H, Abend NS, MacLeod AMK, Sinclair RJ, Snyder AZ, Raichle ME (1999) Tactile attention tasks enhance activation in somatosensory regions of parietal cortex: A positron emission tomography study. Cerebral Cortex 9: 662-674.

Burton H, Sinclair RJ, McLaren DG (2008) Cortical network for vibrotactile attention: A fMRI study. Human Brain Mapping 29: 207-221.

Butter CM, Buchtel HA, Santucci R (1989) Spatial attentional shifts: Further evidence for the role of polysensory mechanisms using visual and tactile stimuli. Neuropsychologia 27: 1231-1240.

Carpenter RHS (1981) Oculomotor procrastination. In: Eye movements: Cognition and visual perception (Fisher DF, Monty RA, Senders JW, eds), pp 237-246. Hillsdale: Lawrence Erlbaum.

Carpenter RHS, Williams MLL (1995) Neural computation of log likelihood in control of saccadic eye movements. Nature 377: 59-62.

Carpenter RHS, McDonald S (2007) LATER predicts saccade latency distributions in reading. Experimental Brain Research 177: 176-183.

Carrasco M, Ling S, Read S (2004) Attention alters appearance. Nature Neuroscience 7: 308-313.

Chica AB, Sanabria D, Lupianez J, Spence C (2007) Comparing intramodal and crossmodal cuing in the endogenous orienting of spatial attention. Experimental Brain Research 179: 353-364.

Cohen J (1988) Statistical power analysis for the behavioral sciences. Hillsdale: Lawrence Erlbaum.

Cohen JC, Bolanowski SJ, Verrillo RT (2005) A direct comparison of exogenous and endogenous inhibition of return and selective attention mechanisms in the somatosensory system. Somatosensory \& Motor Research 22: 269-279.

Craig JC (1999) Grating orientation as a measure of tactile spatial acuity. Somatosensory \& Motor Research 16: 197-206.

Davis R (1957) The human operator as a single channel information system. Quarterly Journal of Experimental Psychology 9: 119-129. 


\subsection{Feature-based attentional modulation of orientation perception in somatosensation}

DiCarlo JJ, Johnson KO (2000) Spatial and temporal structure of receptive fields in primate somatosensory area $3 \mathrm{~b}$ : effects of stimulus scanning direction and orientation. The Journal of Neuroscience 20: 495-510.

Domenech P, Dreher J-C (2010) Decision threshold modulation in the human brain. The Journal of Neuroscience 30: 14305-14317.

Duncan J (1980) The demonstration of capacity limitation. Cognitive Psychology 12: 75-96.

Erdfelder E, Faul F, Buchner A (1996) GPOWER: A general power analysis program. Behavior Research Methods, Instruments, \& Computers 28: 1-11.

Essock EA, Krebs WK, Prather JR (1997) Superior sensitivity for tactile stimuli oriented proximallydistally on the finger: Implications for mixed class 1 and class 2 anisotropies. Journal of Experimental Psychology: Human Perception and Performance 23: 515-527.

Forster B, Eimer M (2004) The attentional selection of spatial and non-spatial attributes in touch: ERP evidence for parallel and independent processes. Biological Psychology 66: 1-20.

Forster B, Eimer M (2005) Covert attention in touch: Behavioral and ERP evidence for costs and benefits. Psychophysiology 42: 171-179.

Fort A, Martin R, Jacquet-Andrieu A, Combe-Pangaud C, Foliot G, Daligault S, Delpuech C (2010) Attentional demand and processing of relevant visual information during simulated driving: $A$ MEG study. Brain Research 1363: 117-127.

Gibson GO, Craig JC (2005) Tactile spatial sensitivity and anisotropy. Perception and Psychophysics 67: 1061-1079.

Hoechstetter K, Rupp A, Meinck H-M, Weckesser D, Bornfleth H, Stippich C, Berg P, Scherg M (2000) Magnetic source imaging of tactile input shows task-independent attention effects in SII. NeuroReport 11: 2461-2465.

Katzner S, Treue S, Busse L (2012) Improving behavioral performance under full attention by adjusting response criteria to changes in stimulus predictability. Journal of Vision 12: 1-13.

Lechelt EC (1988) Spatial asymmetries in tactile discrimination of line orientation: a comparison of the sighted, visually impaired, and blind. Perception 17: 579-585.

Lloyd DM, Bolanowski SJJ, Howard L, McGlone F (1999) Mechanisms of attention in touch. Somatosensory \& Motor Research 16: 3-10.

Oldfield RC (1971) The assessment and analysis of handedness: The Edinburgh inventory. Neuropsychologia 9: 97-113.

Posner MI (1978) Chronometric explorations of mind: Oxford, England: Lawrence Erlbaum.

Reddi BA, Carpenter $\mathrm{RH}$ (2000) The influence of urgency on decision time. Nature Neuroscience 3: 827-830.

Reddi BAJ, Asrress KN, Carpenter RHS (2003) Accuracy, information, and response time in a saccadic decision task. Journal of Neurophysiology 90: 3538-3546.

Rossi AF, Paradiso MA (1995) Feature-specific effects of selective visual attention. Vision Research 35: 621-634.

Saenz M, Buracas GT, Boynton GM (2002) Global effects of feature-based attention in human visual cortex. Nature Neuroscience 5: 631-632.

Saenz M, Buracas GT, Boynton GM (2003) Global feature-based attention for motion and color. Vision Research 43: 629-637. 
Sathian K, Burton H (1991) The role of spatially selective attention in the tactile perception of texture. Perception and Psychophysics 50: 237-248.

Sinclair R, Kuo J, Burton H (2000) Effects on discrimination performance of selective attention to tactile features. Somatosensory \& Motor Research 17: 145-157.

Spence C, Pavani F, Driver J (2000) Crossmodal links between vision and touch in covert endogenous spatial attention. Journal of Experimental Psychology: Human Perception and Performance 26: 1298-1319.

Sperling G (1984) A unified theory of attention and signal detection. In: Varieties of Attention (Parasuraman R, Davies D, eds), pp 103-181. New York: Academic Press.

Treue S, Trujillo JCM (1999) Feature-based attention influences motion processing gain in macaque visual cortex. Nature 399: 575-579.

van Ede F, de Lange FP, Maris E (2012) Attentional cues affect accuracy and reaction time via different cognitive and neural processes. The Journal of Neuroscience 32: 10408-10412.

Vega-Bermudez F, Johnson KO (2001) Differences in spatial acuity between digits. Neurology 56: 1389-1391.

Whang K, Burton H, Shulman G (1991) Selective attention in vibrotactile tasks: Detecting the presence and absence of amplitude change. Perception and Psychophysics 50: 157-165. 


\subsection{Exploration of feature-based somatosensory modulation of responses to orientations: A human fMRI study}

In the present study, correlates of global tactile feature-based attention were explored in an fMRI study with integrated psychophysical task, using orientation as stimulus dimension. While the psychophysical performance pointed to a global feature-based effect, no cortical feature-based attentional effect was observed in SI or SII. The subjects' performance and their cortical activation (representing an unattended location) differed depending on which orientation was attended, pointing to an attentional effect related to processing and perception of specific features. 


\section{Exploration of feature-based somatosensory modulation of responses to orientations: A human fMRI study}

Tactile feature-based fMRI modulation

Meike A. Schweisfurth ${ }^{1,2}$, Stefan Treue ${ }^{2}$, Jens Frahm ${ }^{1}$, Renate Schweizer ${ }^{1}$

1Biomedizinische NMR Forschungs $\mathrm{GmbH}$ am Max-Planck-Institut für biophysikalische Chemie, 37070 Göttingen, Germany

${ }^{2}$ Cognitive Neuroscience Laboratory, German Primate Center, 37077 Göttingen, Germany

Correspondence should be addressed to: Meike Schweisfurth, Biomedizinische NMR Forschungs GmbH am Max-Planck-Institut für biophysikalische Chemie, Am Fassberg 11, 37077 Göttingen, Germany; Email: mschwei1@gwdg.de 


\section{Abstract}

In the present study, correlates of global tactile feature-based attention were explored in an integrated fMRI and psychophysical study.

During each fMRI block, subjects attended (and counted) a previously-cued tactile orientation (either vertical or horizontal) among random orientations at the ring finger, while receiving simultaneous distractor stimulation continuously with either the cued (consistent block) or the perpendicular orientation (inconsistent block) at the index finger. On the behavioral level, miss and false-alarm rates were compared between attentional conditions (vertical / horizontal attended combined with vertical / horizontal distractor). fMRI activation was compared between the 4 attentional conditions for regions of interest (ROIs) in the ring and index finger representations of the primary somatosensory cortex (SI) as well as in left and right SII.

The psychophysical analysis revealed lower miss rates but a trend for higher false-alarm rates for consistent versus inconsistent blocks. No cortical effect of feature-based attention was observed. False-alarm rates were also lower for attention to the vertical compared to the horizontal orientation. This attentional effect was also cortically observed in the index finger's SI ROI, where a higher signal was found for attention to vertical compared to horizontal orientation.

Although the psychophysical differences between consistent and inconsistent blocks showed that subjects were influenced by the feature-based condition, no cortical effect of feature-based attention was observed in SI or SII. For attention to the vertical versus the horizontal orientation, the consistent results at the behavioral and cortical level showed that attentional allocation to features could not only influence the performance of subjects but also their cortical activation.

Keywords: Anisotropy, feature-based attention, fMRI, orientation, psychophysics 


\section{Introduction}

The present study aimed at exploring feature-based attentional effects in the primary and secondary somatosensory cortices. Present in each human sensory system, attention is a process which selectively enhances the information processing of behaviorally-relevant stimuli. Acting like a central bottleneck, it allows for filtering these out of the large amount of sensory influx, thereby strongly influencing the sensory percept of the environment.

In the tactile modality, most attention-exploring imaging studies have been devoted to the comparison between attended and unattended touch. In the attendtouch condition, attention generally is directed to locations and only rarely to objects or stimulus dimensions (e.g. Burton et al., 1999, 2008). For the secondary somatosensory cortex (SII), most (but not all, Galazky et al., 2009) studies observed a higher response in functional magnetic resonance imaging (fMRI) (Burton et al., 2008; Hämäläinen et al., 2000; Johansen-Berg et al., 2000; Nelson et al., 2004; Sterr et al., 2007) or positron emission tomography (PET) (Burton et al., 1999) or a modulated magnetoencephalography (MEG) signal (Mima et al., 1998; Hoechstetter et al., 2000) for attended compared to ignored tactile stimulation. Such attentional effects have also been reported for the contralateral primary somatosensory cortex (SI) by the majority of fMRI (Hämäläinen et al., 2000; Johansen-Berg et al., 2000; Nelson et al., 2004; Staines et al., 2002 ; Sterr et al., 2007) and PET (Meyer et al., 1991; Burton et al., 1999) studies, whereas a few studies did not observe attentional effects in SI (Burton et al., 2008; Galazky et al., 2009; Mima et al., 1998; Hoechstetter et al., 2000). Divergent results concerning the presence or absence as well as the relative size (between SI and SII) of attentional modulations at least partly depend on the exact experimental setting; active distraction from the tactile modality (e.g. backward counting or a visual task) instead of the mere instruction to ignore the stimulation seems to be crucial for finding attentional effects (Johansen-Berg et al., 2000).

Whereas the vast majority of attentional imaging studies in touch so far explored settings related to spatial attention, it has been shown in the visual modality that attention can also be directed to behaviorally-relevant objects or features. Here, a feature is defined as an element of a certain stimulus dimension, red, for example, 

to orientations: A human fMRI study

being one feature within the stimulus dimension of color. In electrophysiological monkey studies, feature-based attention was deduced from the observation that attending to a certain feature (e.g. direction of motion, color) increased the spike rate of those neurons whose preferred feature matched with the attended feature and decreased the spike rate of those cells preferring the 'opposite' feature (e.g. the opposite motion direction) (Treue and Martinez-Trujillo, 1999). Most interestingly, these effects are observable even if the attended feature is presented far outside the neuron's receptive field, revealing a (spatially) global effect of feature-based attention. These feature-based effects are explained by the feature-similarity gain model (Treue and Martinez-Trujillo, 1999; Martinez-Trujillo and Treue 2004), which in its essence describes the sign and magnitude of attentional gain modulation of a neuron depending on the similarity between the presently attended feature and the neuron's own feature preferences (Maunsell and Treue, 2006). Global feature-based attention has also been shown in the human visual system. Psychophysically, it was shown to improve behavior (Rossi and Paradiso, 1995; Saenz et al., 2003). On the cortical level, numerous imaging studies could demonstrate feature-based attentional effects and confirm the feature-similarity gain model for different stimulus dimensions. Several studies consistently found increased fMRI responses for attended versus unattended features for the stimulus dimension of motion direction (Saenz et al., 2002; Serences et al., 2006; Castelo-Branco et al., 2009; Stoppel et al., 2011). In the Saenz study, for example, subjects attended to a location where two opposite motion directions were simultaneously presented, but were instructed to focus on just one of these directions, for which speed changes should be detected. Simultaneously, one of these two motion directions was presented at a distant, behaviorally-irrelevant location. Interestingly, the fMRI signal found in the cortical representation of the distractor location was higher, if the distantly attended and the locally presented motion directions were consistent compared to if they differed, showing an effect of global feature-based attention. Exploring the stimulus dimension of grating orientation, Liu and coworkers (2007) found more-pronounced adaptation due to feature-based orienting (during identical sensory input), as lower fMRI responses were observed to orientation-matched compared to orientationunmatched test stimuli presented shortly after adaptation. 
Despite its thorough investigation in the visual modality, feature-based attention has hardly been explored in the tactile domain. While previous studies explored attention to entire stimulus dimensions (Burton et al., 1999, 2008; Hoechstetter et al., 2000; Sinclair et al., 2000), these studies did not investigate what we define as feature-based attention, i.e. the direction of attention to one out of several features within the same stimulus dimension. Only one ERP (event-related potential) study by Forster and Eimer (2004) and one reaction-time study by our group (Schweisfurth et al., in preparation) have addressed the issue of tactile feature-based attention and delivered first cortical and behavioral indications for global feature-based attention in touch.

For this study, orientation was chosen as stimulus dimension. First, effects of orientation-based attention were observable in a recent behavioral study (Schweisfurth et al., in preparation) and should hence have potential cortical correlates that remain to be explored. Secondly, literature suggests that orientation processing in the primary and secondary somatosensory cortices might be modulated in response to feature-based attention. Monkey studies (Hsiao et al., 2002) revealed that tactile orientation processing begins with activation of slowly adapting type 1 (SA1) fibers (Johnson and Hsiao, 1992), which densely innervate the fingertip. Although each of these on its own is hardly orientation-sensitive (Bensmaia et al., 2008a), their population response reliably encodes the presented orientation (Khalsa et al., 1998). Via the thalamus, the information is passed on to SI and then SIl (Pons et al., 1987), for both of which a pronounced amount of orientationsensitive neurons were identified (for SI more than 50\%, Bensmaia et al., 2008a, and for SII about 20\%, Fitzgerald et al., 2006). Further on, the most orientation-sensitive neurons in the monkey study in SI presented with angular thresholds low enough to account for the psychophysical performance in humans (Bensmaia et al., 2008a,b). Hence, SI fulfils all requirements for being an area potentially modulated by tactile orientation-based attention, similar as medial temporal area (MT) for attention to visual motion directions (Treue and Martinez-Trujillo, 1999).

In the present human study, we intended to get a first insight into whether and where feature-based attention can be found in the tactile domain. Therefore, we transferred the experimental paradigm of the visual feature-based fMRI study by Saenz and coworkers (2002) with some adaptations into the somatosensory domain. 

to orientations: A human fMRI study

Random tactile orientations were presented to the subjects' ring finger, forming the attended location. To direct the subjects' attention to a certain orientation there, they were instructed to attend to that orientation at the ring finger and count how often it was presented there. Simultaneously, the index finger (forming the distractor location) received stimulation persistently with either the subjects' attended (consistent block) or the perpendicular (inconsistent block) orientation; both were behaviorally irrelevant for the subjects and should be ignored. Our main interest was then to explore whether attending to an orientation at the attended location led to improved processing of that orientation even at the spatially distant, featureirrelevant location, i.e. whether we could find evidence for global feature-based attention as observed in vision. 


\section{Materials and Methods}

Approval for the present study was obtained from the Ethics Committee of the Georg-Elias-Müller-Institut für Psychologie, Göttingen. The 13 healthy subjects (5 women, range 23 to 30 years, mean $26 \pm 3$ years) participated in 4 magnetic resonance imaging (MRI) sessions, giving their informed written consent before each session. They all were right-handed according to the Edinburgh Inventory (laterality index $0.9 \pm 0.1$, minimum 0.7; Oldfield, 1971) and all had taken part in a previous psychophysical study on tactile feature-based attention (Schweisfurth et al., in preparation).

\section{Magnetic resonance imaging}

For MRI, a 3 T-system (TIM Trio, Siemens Healthcare, Erlangen, Germany) equipped with a 32-channel head coil was used. In the first session, our standard somatosensory warm-up routine was run, including a high-quality structural MR image, a MR angiography, a whole-brain echo-planar image (EPI) as well as a highresolution functional localizer measurement for locating the digit representations in the left primary somatosensory cortex $(\mathrm{SI})$. In the second session, two identical further digit-representation functional-localizer measurements covered the digit area of SI and SII of both hemispheres. Also, a standard structural image was acquired. The third and fourth imaging session each consisted of $5 \mathrm{fMRI}$ measurements in which the subject had to perform the below described task.

The high-quality structural image was acquired using a sagittal T1-weighted 3D MPRAGE (magnetization-prepared rapid gradient-echo) sequence (repetition time $(\mathrm{TR})=2530 \mathrm{~ms}$, echo time $(\mathrm{TE})=3.4 \mathrm{~ms}$, flip angle $=7^{\circ}$, acquisition matrix $=$ $256 \times 256, \quad 160-192$ partitions, resolution $=1 \times 1 \times 1 \mathrm{~mm}^{3}$, total acquisition time $(T A)=10: 49 \mathrm{~min})$. The standard structural image was recorded using a sagittal T1-weighted 3D FLASH (fast low angle shot) sequence (repetition time $(T R)=$ $10.55 \mathrm{~ms}$, echo time $(T E)=4.24 \mathrm{~ms}$, flip angle $=17^{\circ}$, acquisition matrix $=256 \times 256$, $160-192$ partitions, resolution $=1 \times 1 \times 1 \mathrm{~mm}^{3}$, acceleration factor $($ GRAPPA $)=2$, total acquisition time $(T A)=3: 36-4: 19 \mathrm{~min})$.

Functional imaging of sessions 2 to 4 was performed with a gradient-echo EPI sequence at $2 \times 2 \times 4 \mathrm{~mm}^{3}$ resolution $\left(\mathrm{TR}=2000 \mathrm{~ms}, \mathrm{TE}=36 \mathrm{~ms}\right.$, flip angle $=70^{\circ}$, 
acquisition matrix $=96 \times 96$, field of view $=192 \times 192 \mathrm{~mm}^{2}$, partial Fourier factor $=$ 7/8). The 22 oblique transverse-to-coronal sections were positioned such that the whole volume from above the motor hand knob at the precentral gyrus (Yousry et al., 1997) to below the parietal operculum (Eickhoff et al., 2005a, Eickhoff et al., 2005b) was covered. A very similar slice position for all attentional fMRI runs was achieved by applying the AutoAlign Scout (Siemens Healthcare, Erlangen, Germany) before each run.

Except for the high-quality anatomy, the warm-up session measurements were only used to check that subjects exhibited the expected medial-to-lateral digit somatotopy in SI (e.g. Penfield and Rasmussen, 1950; Schweizer et al., 2008) and that they hence could be included in further sessions. All these measurements were therefore positioned such that the digit area in SI was covered. High-resolution functional images were acquired using a gradient-echo EPI sequence with $1.5 \times 1.5 \times 1.5 \mathrm{~mm}^{3}$ resolution $\left(\mathrm{TR}=2000 \mathrm{~ms}, \mathrm{TE}=36 \mathrm{~ms}\right.$, flip angle $=70^{\circ}$, acquisition matrix $=128 \times 128$, field of view $=192 \times 192 \mathrm{~mm}^{2}$, partial Fourier factor $=$ $6 / 8,19$ slices). The same orientation, resolution, and whole-brain volume coverage was used for the single multi-slice EPI acquisition $\left(1.5 \times 1.5 \times 1.5 \mathrm{~mm}^{3}\right.$ resolution, $\mathrm{TR}=8600 \mathrm{~ms}, \mathrm{TE}=36 \mathrm{~ms}$, flip angle $=70^{\circ}$, acquisi tion matrix $=128 \times 128$, field of view $=192 \times 192 \mathrm{~mm}^{2}$, partial Fourier factor $=6 / 8,81$ sections). For MR angiography, a T1-weighted 3D FLASH sequence was used $(T R=22 \mathrm{~ms}$, $\mathrm{TR}=4.43 \mathrm{~ms}$, flip angle $=18^{\circ}$, resolution $=0.3 \times 0.3 \times 0.5 \mathrm{~mm}^{3}, 57$ sections from two overlapping slabs), comprising with the same volume coverage and orientation as the functional images. Paradigm and evaluation of these measurements have been described previously (Schweisfurth et al., 2011). No subjects had to be excluded based on the warm-up session measurement.

\section{Tactile stimulation}

For tactile stimulation, two systems (QuaeroSys, St. Johann, Germany) were used, both consisting of a control unit along with independent piezo-electric stimulation modules. These modules comprised with Braille displays (consisting of individually raisable pins) at their proximal surfaces and could be positioned below individual fingertips, such that the Braille display was located centrally below the fingertip with the bulk of the module pointing away from the digit. 
Using cushions to adjust the relative position between palm, digits, and modules, it was ensured that subjects could keep their stimulated hand relaxed and pronated. In order to achieve maximal stimulation strength, pins were always elevated with maximal possible force, resulting in a stick-out height of about $1.5 \mathrm{~mm}$ if no weight was applied onto them.

For the functional localizer (measured in the first and second MRI session), the fingertips of all right-hand digits were stimulated using 5 stimulation modules with an 8-dot Braille display $\left(2 \times 4\right.$ matrix, covering an area of $2.5 \times 7.5 \mathrm{~mm}^{2}$; Schweizer et al., 2008; Schweisfurth et al., 2011). During stimulation blocks, fast-varying

Figure 1. Stimulation. (A) Tactile radial stimulator. (B) Illustration of the 4 possible orientation patterns and their location relative to the proximal-to-distal fingertip axis. (C) Actually presented realizations of orientation, exemplified with the vertical orientation.
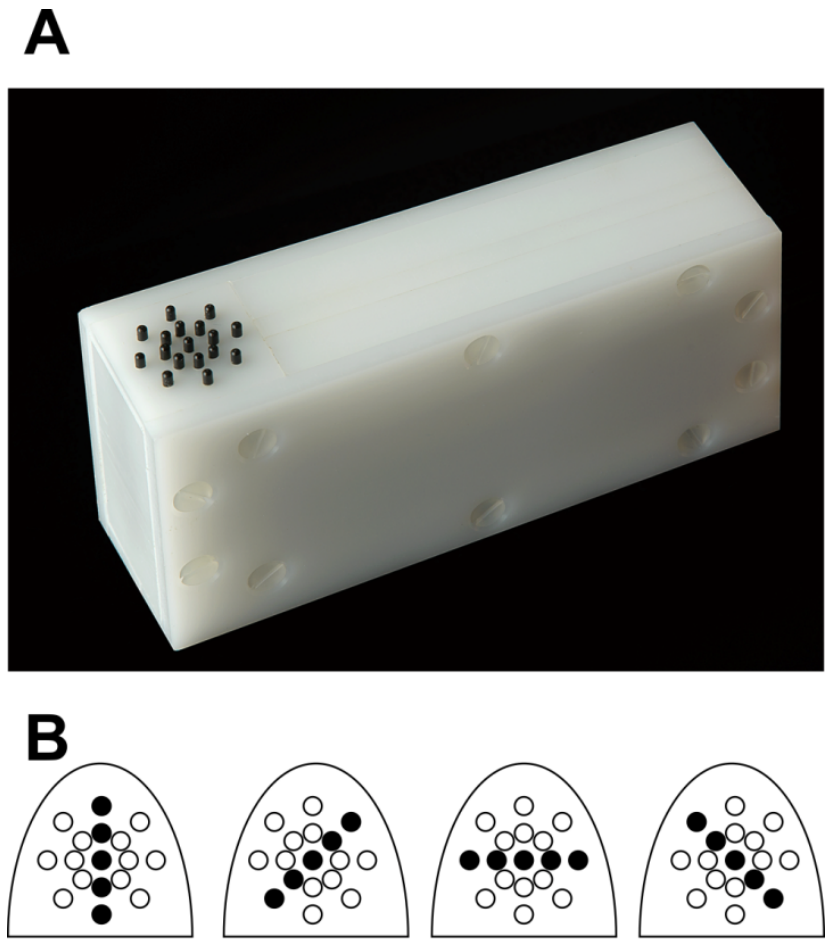

vertical
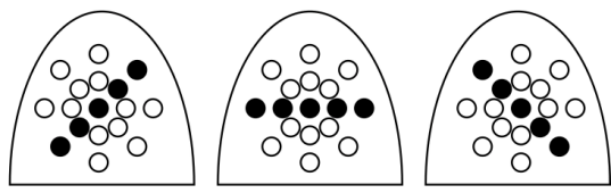

diagonal horizontal diagonal

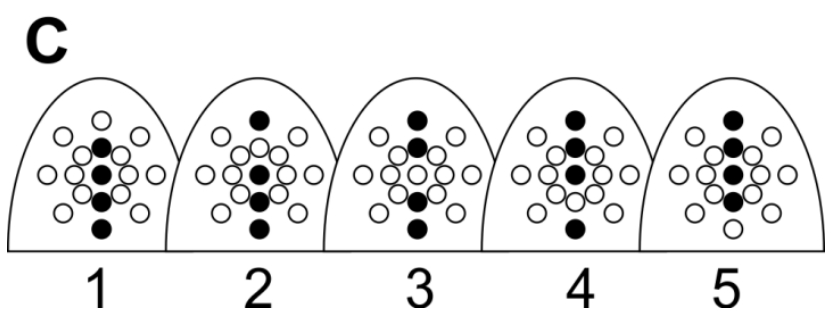
stimulation patterns (frequency of $32 \mathrm{~Hz}$ ) were displayed across the entire Braille display, by randomly choosing and elevating two pins per cycle (square wave, uptime = $10.4 \mathrm{~ms}$, downtime $=20.8 \mathrm{~ms}$ ). To control that subjects focused onto the stimulation, they should covertly count short randomly distributed interrupts during stimulation blocks (duration = $156 \mathrm{~ms}$, occurring every 0.5 to $3 \mathrm{~s}$ ) and verbally report (after the measurement) the number of interrupts to the experimenter who then gave feedback about the quality of the count. The experimental design of the functional localizer was as follows: Stimulation went sequentially along all right-hand fingertips (from thumb to little finger, $12 \mathrm{~s}=6$ images each) with a rest period (12 $\mathrm{s}=$ 6 images) placed after each fourth digit stimulation (D1, D2, D3, D4, Rest, D5, D1, D2, D3, Rest, D4, D5, 
D1, D2...). As each digit was stimulated 8 times (96 s, 48 images), a total scan time of 10:20 min (310 images) including baseline (20 s, 10 images) was needed.

For the attentional runs (third and fourth MRI session) used for feature-based analysis, the fingertip of the right index (D2) and ring (D4) finger were stimulated. Custom-built stimulation modules comprised a 17-dot radial Braille display (one central pin, two surrounding rings à 8 pins each, having a radius of $2.5 \mathrm{~mm}$ and $5 \mathrm{~mm}$, respectively, Fig. 1A). By simultaneously rising pins along the same spoke, each radial stimulator could present 4 different orientations: Vertical, horizontal, und the two diagonals, each one presented by 5 linearly aligned rods (Fig. 1B). In order to prevent subjects from identifying an orientation by specific pins instead of their common configuration, always only 4 of the 5 aligned pins (called the chosen realization of orientation) were raised whenever an orientation was to be presented (Fig. 1C). Before each fMRI measurement, the modules were positioned below the respective fingertip such that the vertical stimulator orientation was in line with the proximal-to-distal fingertip axis. Stimulation frequency was set to $10 \mathrm{~Hz}$ (stimulus duration $=50 \mathrm{~ms}$, inter-stimulus interval $=50 \mathrm{~ms}$ ).

A blocked design was used for the attentional runs (Fig. 2A), preceded by 4 preparation scans $(8 \mathrm{~s})$ and 6 baseline images $(12 \mathrm{~s})$. Each run (266 images $=$ 8:52 min) included 16 blocks consisting of a cue ( 1 image $=2 \mathrm{~s})$, a stimulation $(7$ images $=14 \mathrm{~s})$, a response $(2$ images $=4 \mathrm{~s})$, and a rest period $(6$ images $=12 \mathrm{~s})$. In each stimulation period, 18 tactile orientations were sequentially presented, simultaneously at the two fingers (Fig. 2C): At D4 each orientation was randomly chosen between the 4 possible, while at D2 all presented orientations in a block were either horizontal or vertical (distractor orientation). Within presentation of one orientation, the same orientation realization was presented 5 times, leading to a stimulation length of $500 \mathrm{~ms}$ per orientation. Orientations were separated by a $278 \mathrm{~ms}$ inter-pulse interval. Two seconds before each stimulation block a tactile taskinformative cue with either horizontal or vertical orientation was presented at D4, realized by 5 -times elevating all pins for the respective orientation. Subjects were supposed to ignore the index finger (so-called distractor location) but concentrate on the ring finger (so-called attended location) and count how often the attended orientation was presented there during the entire block. 


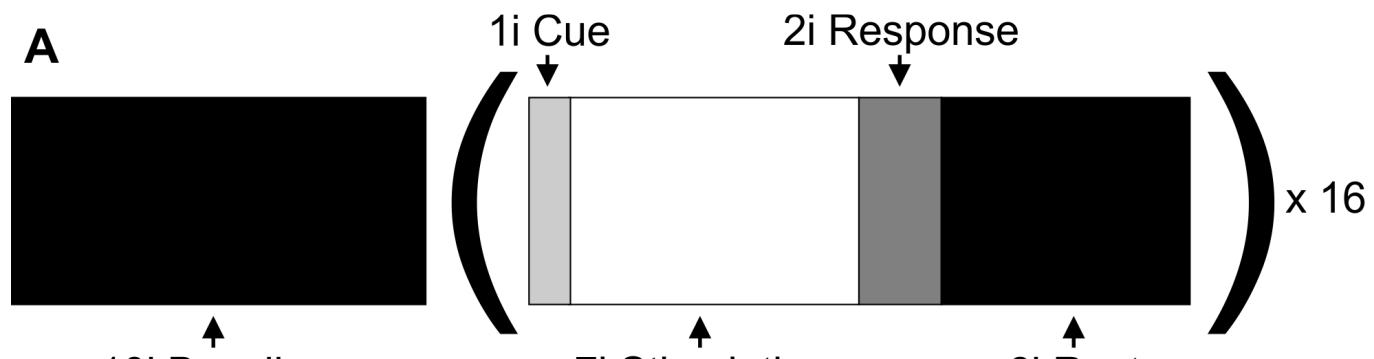

10i Baseline

7i Stimulation

6i Rest

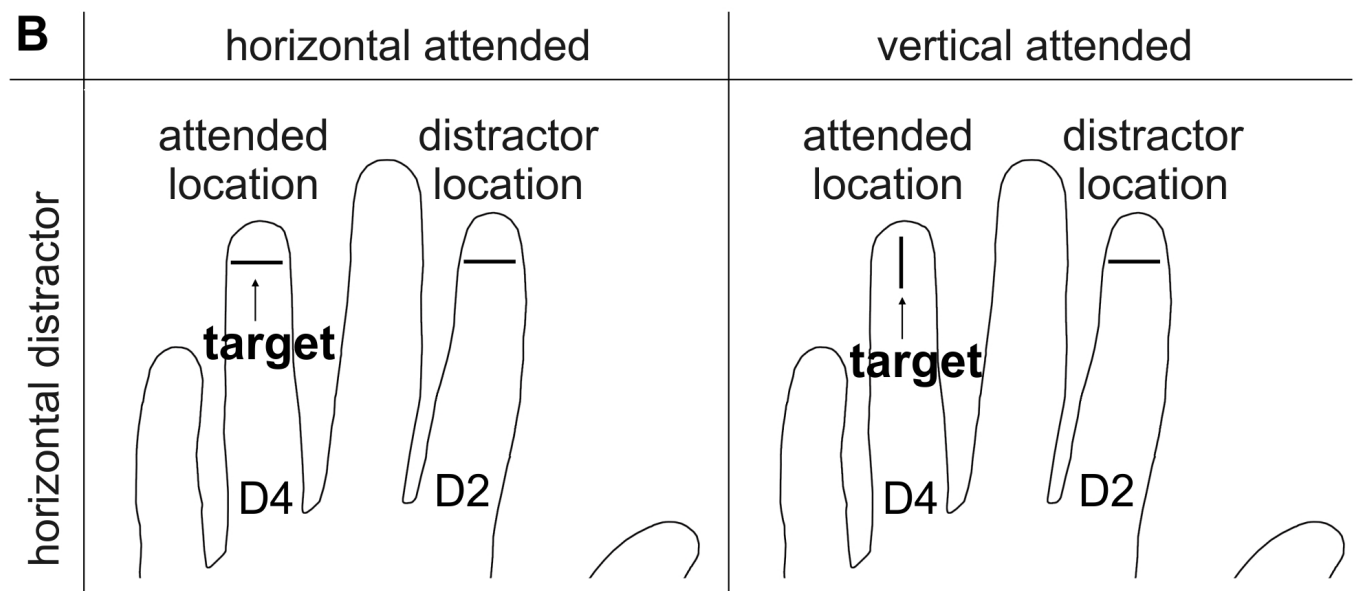

inconsistent block

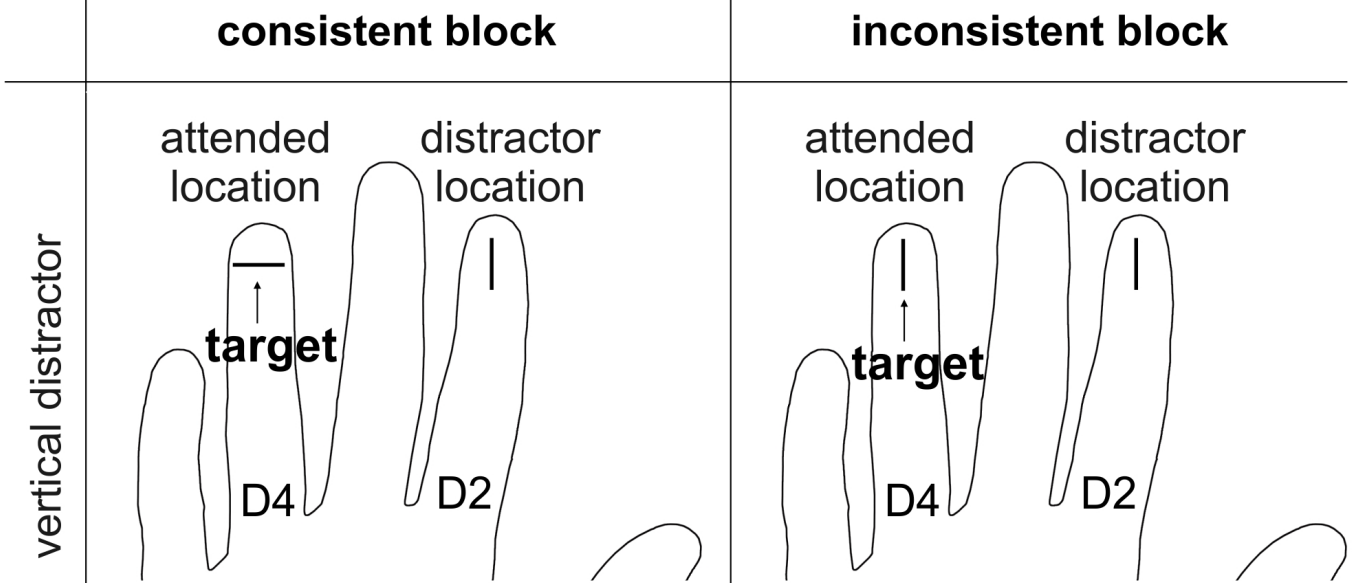

inconsistent block

\section{consistent block}

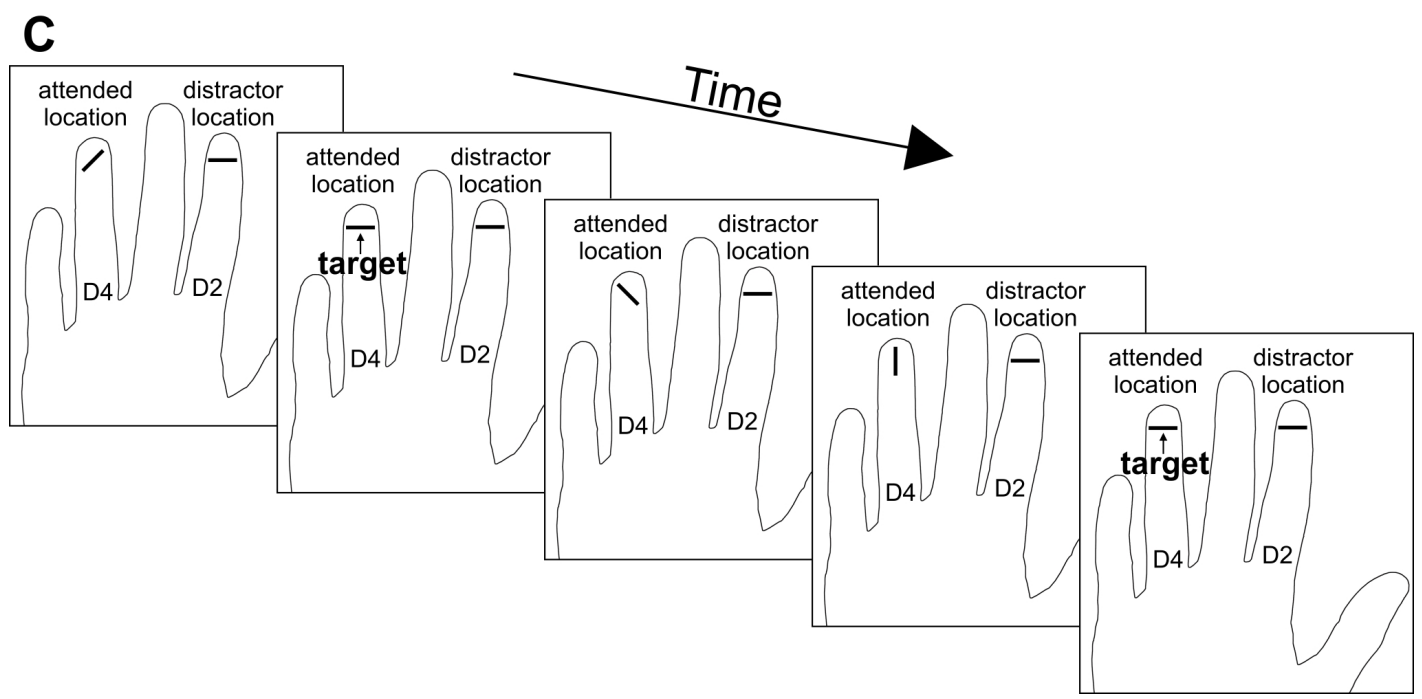


During the response period (4s after each stimulation period), subjects reported whether their count had been lower or higher than (or equal to) 5 , using the left or right foot pedal, respectively. Depending on whether the distractor orientation (vertical or horizontal, continuously presented at D2) and the attended orientation (vertical or horizontal, cued at D4 and then presented there with 25\% probability per presented orientation) in a block differed or not, the block was called inconsistent or consistent (Fig. 2B).

Subjects were familiarized with and trained in the attentional paradigm after the second session (one to three training runs) and before both attentional sessions (one training run).

\section{Preprocessing and co-registration}

Initial data analysis was carried out mainly using BrainVoyager QX 2.4 (Goebel et al., 2006, Brain Innovation, Maastricht, The Netherlands). Each subject's high-quality structural image (first session) was corrected for intensity inhomogeneities and, using rigid body transformations, transferred into ACPC space (i.e. translated and rotated to obtain axial slice orientation parallel to the plane through anterior and posterior commissures), using sinc interpolation. The second session's standard structural image of the subject was then registered ( 6 degrees of freedom, sinc interpolation) to the described ACPC image (creating a standard-tohigh-quality-ACPC matrix). All functional runs of the second to fourth session were motion-corrected in k-space (online Siemens software) and in image space (BrainVoyager) as well as temporally

Figure 2. Positioning and paradigm. (A) Run design. Each run consists of a baseline period followed by 16 blocks each consisting of periods of cue presentation, stimulation, response, and rest. (B) Block types. During the cueing period, a vertical or horizontal orientation is presented solely at D4 and is to be attended there throughout the block. During the stimulation period, D4 receives stimulation of random orientation, whereas D2 receives either only vertical or only horizontal stimulation. If the orientations attended at D4 and presented at D2 (dis-)agree, the block is referred to as (in-)consistent. (C) Exemplary cutout of a consistent-block timecourse with horizontal orientation: While D2 stimulation is horizontal throughout the block, random orientations are presented at D4; only horizontal orientations are to be attended and should be counted. high-pass filtered as implemented as default in BrainVoyager. Importantly, all runs of a subject were registered to the first functional localizer of the second session (using trilinear/sinc interpolation) to achieve optimal coregistration between runs. For each subject, this functional localizer was then registered $(6$ degrees of freedom) to the second session's 
individual structural image (using the header information and additional manual optimization). The obtained registration matrix was concatenated with the standardto-high-quality-ACPC matrix and then applied to all runs of the subject (using trilinear interpolation), allowing for identical projection onto the subject's anatomy. In this step, fMRI images were interpolated from $2 \times 2 \times 4 \mathrm{~mm}^{3}$ resolution to $2 \mathrm{~mm}$ isotropic resolution. The applied registration approach allowed for precise coregistration both between functional runs and between functional and structural data.

\section{ROIs, digit somatotopy, and digit anisotropy}

Four regions of interest (ROls: "Left SI, D2"; "Left SI, D4"; "Left SII", "Right SII") were automatically defined for each subject separately, taking into account the Jülich atlas as well as the subject's anatomy and 5-digit functional localizer (see Fig. 3).

From the two second-session functional localizer runs, the individual fingerrepresentation map was obtained by calculating the main effect of the finger's predictor (being convoluted with a two-gamma function, Friston et al., 1998) in a global general linear model (GLM) analysis (fixed-effects analysis across the two runs). Voxels with higher $\mathrm{t}$-value than the one corresponding to a false discovery rate of $\mathrm{q}(\mathrm{FDR})=0.05$ were considered significant. Further on, a conjunction map was calculated for these 5 main effects, reflecting voxels activated for all 5 fingers.

In order to use the Jülich atlas (Eickhoff et al., 2005), FSL (FMRIB Software Library, Smith et al., 2004) was used to define anatomical regions of interest (ROIs) which were then imported into BrainVoyager. In FSL, out of the areas available for the standard brain (two-millimeter isotropic Montreal Neurological Institute (MNI)) in the Jülich atlas (FSL Anatomy Toolbox, version 1.8), the regions of the left primary somatosensory cortex (left BA 3a, 3b, 1, and 2) as well as those regions of both parietal opercula which are presumable involved in somatosensation (left and right OP1 and OP4, Eickhoff et al., 2006b) were chosen at a level of $30 \%$ probability, i.e. including the volume where the respective region was identified in at least 3 out of the 10 explored subjects. This large volume was chosen in order to allow for individual anatomical deviations. Then, the following steps were carried out for all subjects. First, their high-quality anatomical image was loaded in FSL and nonlinearly registered (using FNIRT, FMRIB's nonlinear image registration tool) to the 
standard brain, generating a warp matrix. Then, the inverse warp matrix was applied to all above-defined anatomical standard-space ROls, leading to ROls registered to the individuals' high-quality anatomical image.

To achieve ACPC-registered ROIs, the ACPC high-quality image was exported from BrainVoyager into FSL. Using FLIRT (FMRIB's Linear Image Registration Tool, Jenkinson and Smith, 2001), the FSL-generated high-quality image was registered to the BrainVoyager-generated high-quality ACPC image and the respective matrix was then applied to the generated ROls. Now, the ROls for the left SI (BA 3a, BA 3b, BA 1, and BA2) as well as the ones for left and right SII (respective OP1 and OP4) were pooled, flipped into BrainVoyager orientation, imported into it, and defined as individual anatomical ROIs.

Combining the information from these anatomical ROls and the functional localizer, for each ROI separately the coordinates of each voxel along with its t-values for each individual digit map as well as the one for the conjunction map were exported into MATLAB. There, anatomical-functional ROls were defined as follows. For right and left SII (see Fig. 3A), all voxels (within the respective anatomical ROI) significant in the conjunction map were assessed and among them, the statistically most active voxel was determined. "Left SII" and "Right SII" were then defined as this peak voxel along with its 6 immediate neighbors. For the attentional analysis, two SI ROIs were defined in a three-step procedure (see Fig. 3B), one for the index and one for the ring finger representation. For the definition of "Left SI, D4", first, within the anatomically defined ROI the significant voxels of the D4-map were identified. Out of these, all voxels also significant in the conjunction map were excluded in a second step. Out of the remaining voxels, the peak voxel along with its 6 neighbors was then defined as the final ROI "Left SI, D4". Analogously, "Left SI, D2" was defined. These definitions were based on the assumption that finger representations in $\mathrm{SI}$ should not comprise with areas where all 5 fingers are represented (if observed,

Figure 3. ROI-generation process. (A) Generation of "Left SII". The FSL-imported Left-SII Jülich-ROI (firstrow image) was intersected with the localizer all-digit conjunction map (second-row image); peak voxel and neighbors were then defined as "Left SIl" (black cross in third-row image). (B) Generation of "Left SI, D4". The FSL-imported Left-SI Jülich-ROI (first-row image) was intersected with the individual localizer D4-map (second-row image); then, the localizer all-digit conjunction map (third-row image) was subtracted. "Left SI, D4" (black cross in forth-row image) was then defined as the peak voxel and its neighbors within the remaining map. $A=$ anterior, $I=$ inferior, $L=$ lateral, $M=$ medial, $\mathrm{P}=$ posterior, $\mathrm{S}=$ superior. 
A ROI generation: Left SII
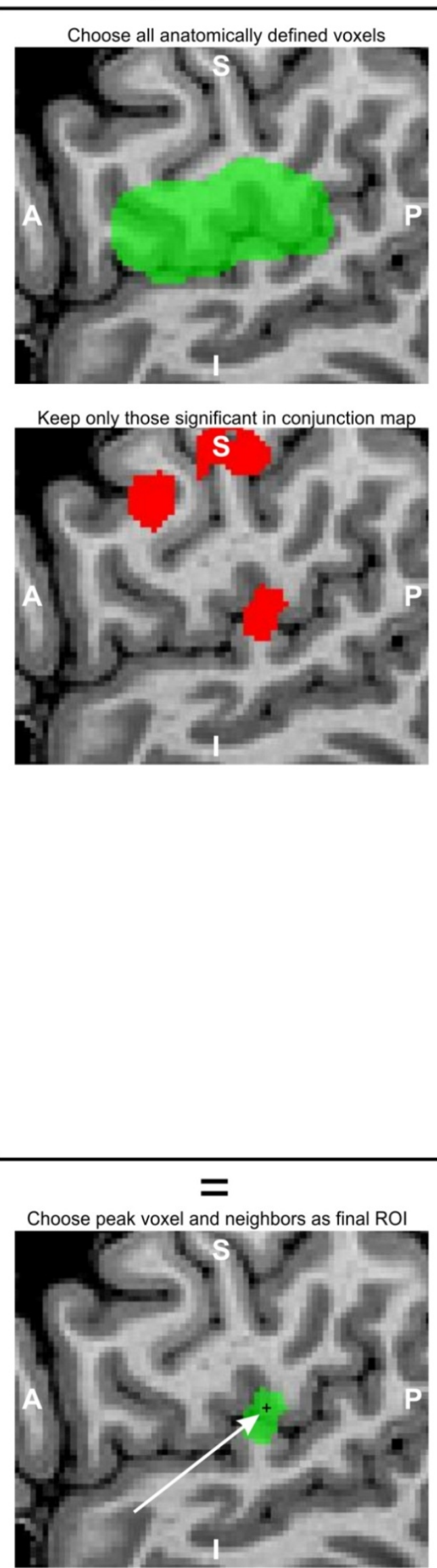

B ROI generation: Left SI, D4

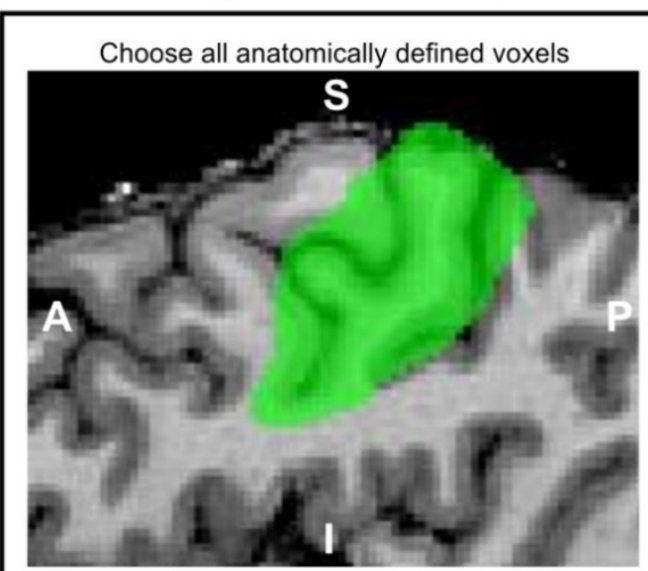

Keep only those significant in D4-map

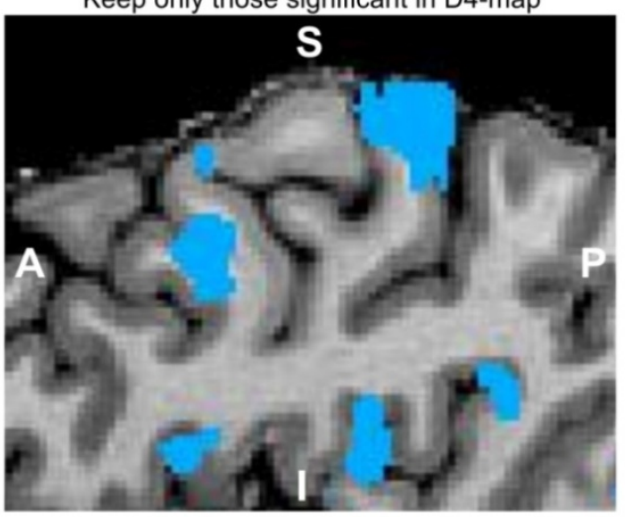

Exclude voxels significant in conjunction map

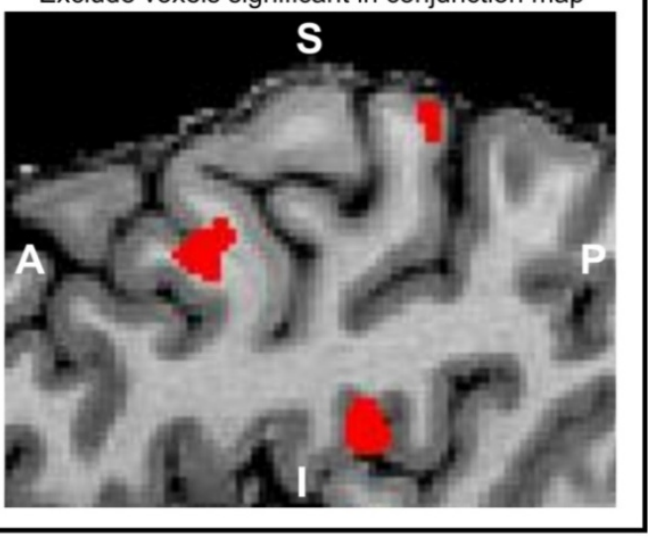

$=$

Choose peak voxel and neighbors as final ROI

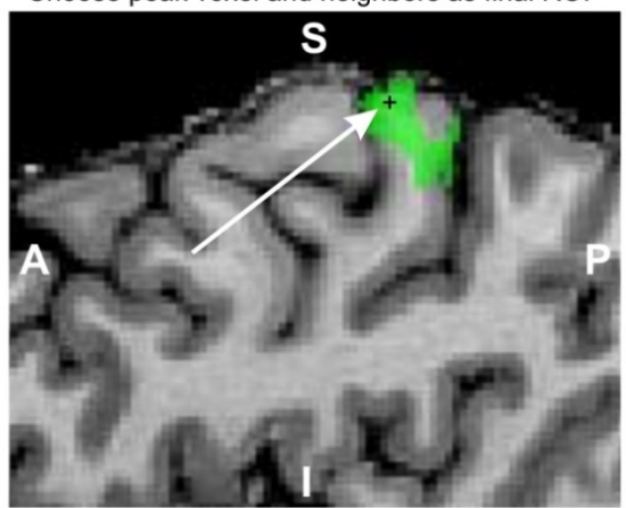


these rather are vessels, at least in Brodmann areas (BAs) 3b and 1; Schweisfurth et al., submitted), whereas in SIl overlapping or even co-located finger representations are expected, at least at the resolution used here (Gelnar et al., 1998; Ruben et al., 2001). The peak-voxel approach was chosen because taking into account all voxels did not allow for sufficient separability between conditions and between patterns in the two SI ROIs (very similar time courses are then observed for these; data shown in Suppl. Fig. S1). For all subjects, the 4 newly-defined ROls were imported back into BrainVoyager and used for all further analysis.

To show the suitability of the ROI-selection routine, left-SI ROls were additionally determined for the remaining digits, analogously to "Left SI, D2" and "Left SI, D4". That allowed for exploring whether the digit ROls showed the expected SI-somatotopy from little finger to thumb (e.g. Penfield and Rasmussen, 1950; Schweizer et al., 2008). For each ROI, the percent BOLD signal change of the functional localizer scans was calculated ${ }^{1}$ for each subject and each digit, mainly to compare the activation between the two SI ROls and between the two SII ROls (using two-tailed paired t-tests, Bonferroni-corrected for the two tests).

\section{Attentional analysis: Psychophysics}

For each subject, the average success, failure, and ignore rates across runs were calculated (number of correct / wrong / no response blocks divided by number of blocks, respectively).

In a second analysis step, the failure rate was determined (taking into account only blocks with given response) separately for each of the 4 attentional conditions (attention on vertical / horizontal orientation at D4 paired with vertical / horizontal distractor at D2) and separately for blocks where more than 4 (high target quantity) / less than 5 (low target quantity) targets (i.e. attended orientations) were presented at D4. These failure rates were compared in IBM SPSS (Statistical Product and Service Solutions) Statistics with a three-way repeated measures analysis of variance (RM ANOVA) with factors "attended orientation", "distractor orientation", and "target quantity". Significant interactions were broken down by two-way RM ANOVA and

${ }^{1}$ For that calculation, the BrainVoyager 2.6 VOI GLM tool included in the "Volumen-of-Interest Analysis" GUI was used, as we could show that the formerly-used BrainVoyager 2.4 tool revealed different results for specific analysis procedures that ought to deliver the same results. 
remaining interactions by further simple-effects analysis (i.e. paired two-tailed t-tests, corrected for in total 4 post-hoc tests).

\section{Attentional analysis: fMRI}

For the attentional runs (third and fourth sessions), fMRI analysis was restricted to the above-defined regions of interest (ROIs), as at most small differences were expected between conditions and too much would be lost due to multiple-comparison correction and inter-subject variability in a whole-brain group analysis (Johansen-Berg et al., 2000).

For each of these ROls, event-related average (ERA) analysis was performed in BrainVoyager $2.6^{2}$ combined with MATLAB, assessing whether the fMRI signal differed between the 4 combinations of attended orientation and distractor orientation. For each ROI, the ERA was calculated for each condition for each run and then averaged across runs for each subject. For each condition, the average percent BOLD signal change (similar to Castelo-Branco et al., 2009) was then calculated for each subject, taking into account the 6 final images (so-called analysis time-window) acquired during stimulation to consider the BOLD latency (shift by one image) and to prevent analysis of the final image, because close to the end of a stimulation block subjects might already have counted sufficient (more than 4) target orientations to decide that they should press the right button. Hence, the final image might have been subject to lower degree of concentration as well as motor preparation for the foot response.

Pooling across conditions for each $\mathrm{ROI}$, it was tested whether the average BOLD response in the analysis time-window differed from zero (two-tailed onesample t-test, corrected for the $4 \mathrm{RO} / \mathrm{s})$. We also explored whether the activation level differed between the two SI or the two SII ROls (using paired two-tailed t-tests, Bonferroni-corrected for the two tests).

\footnotetext{
${ }^{2}$ More precisely, the BrainVoyager 2.6 event-related average (ERA) tool included in the "Volumen-ofInterest Analysis" GUI was used. However, we detected that individual assessment of ERAs (by choosing only the subject's specific ROls and hence calculating the ROI time courses for only one subject at a time) led to different time courses than choosing all ROls at a time; the latter procedure was originally suggested by the developers. Upon our request, the BrainVoyager team could reproduce the finding and, in agreement with our own analysis (comparing several analysis features in BrainVoyager), recommended to use our individual-assessment approach, which now has been used for analysis here.
} 
2.4 Exploration of feature-based somatosensory modulation of responses to orientations: A human fMRI study

To explore potential differences between the 4 attentional conditions, their average percent BOLD signal changes were compared by two-way RM ANOVA (corrected with 4 for the tests in 4 ROls), assessing the effects of "attended orientation", "distractor orientation", and their interaction. 


\section{Results}

Subjects participated in a 4-session fMRI experiment devoted to the exploration of tactile feature-based attention at the cortical level. While attending to tactilely presented targets of a certain orientation at the ring finger (where random orientations were presented), they received stimulation with either the attended or the perpendicular orientation at the index finger. Subjects presented with appropriate psychophysical performance that turned out to depend on feature-based conditions. In the automatically-determined SI and SII ROIs, pronounced BOLD responses to stimulation could be observed. For the SI ROI associated with the ignored index finger, a higher signal was found if subjects attended to vertical compared to horizontal targets at the ring finger, independent of the distractor orientation presented at the index finger.

\section{ROIs, digit somatotopy, and digit anisotropy}

The 4 regions of interest used for attentional analysis (ROIs: "Left SI, D2", "Left SI, D4", "Left SII", "Right SII") as well as the additional ROls for localizer analysis (ROIs: "Left SI, D1", "Left SI, D3", "Left SI, D5") were automatically defined for each subject separately, taking into account the Jülich atlas, the subject's anatomy and the subject's 5-digit functional localizer.

Visual inspection showed that the postcentral sulcus was entirely included in the individual anatomical ROIs (see Fig. 3B), indicating that no SI activation should have been missed. For the SII ROIs, the same conclusion was drawn by visual comparison with the respective ROIs in the standard brain in FSL.

Assessing the peak-value coordinates of the 5 digit ROIs in SI (see Suppl. Fig. S2, panel A-C), a lateral-to-medial, anterior-to-posterior, and inferior-to-superior digit succession from thumb to little finger was observed. Also, the strength of the BOLD signal decreased from D1 to D5 (see Suppl. Fig. S2D). For the attentional ROls, the mean ROI sizes before peaking as well as the percent BOLD signal change of the final $\mathrm{ROI}$ (consisting of peak voxel and neighbors) in the respective localizer map are stated in Table 1. Significantly higher ROI activation (percent BOLD signal change) was observed in this functional localizer for D2 stimulation compared to D4 stimulation (paired two-tailed t-test, mean distance in beta 
value $=0.71$, standard error $=0.26, \mathrm{t}(12)=2.7, \mathrm{p}=0.019$ ). Also, higher activation was observed in the left compared to right SII ROI (mean distance in conjunctionmap beta value $=0.51$, standard error $=0.09, \mathrm{t}(12)=5.9, \mathrm{p}<0.001$ ) .

All further $\mathrm{fMRI}$ analyses performed in this manuscript were restricted to the above-defined 4 attentional ROls consisting of the peak voxel and its 6 neighbors.

\section{Attentional analysis: Psychophysics}

All subjects claimed to understand the task. Pooling across attentional conditions, success, failure, and ignore rates of $80 \pm 8 \%$ (mean \pm standard deviation), $19 \pm 8 \%$, and $1 \pm 1 \%$ were observed, showing that the target difficulty was appropriate and that subjects almost always reacted in the required time window. This high level of performance was expected, as all subjects had taken part in a previous purely psychophysical experiment (Schweisfurth et al., in preparation) where a response was required upon detection of horizontal or vertical targets and hence were well-trained for orientation sensing at their fingertips.

The failure rates for different conditions are listed in Table 2 (see also Fig. 4). The three-way RM ANOVA (see Table 3A) revealed a higher failure rate for blocks with high compared to low target quantity, showing that subjects - as expected - more commonly missed a target than mistook a non-target orientation as target. Also, a higher failure rate was observed for attention to horizontal compared to vertical orientation. As the three-way interaction was significant, separate two-way RM ANOVAs were calculated for the two target quantities.
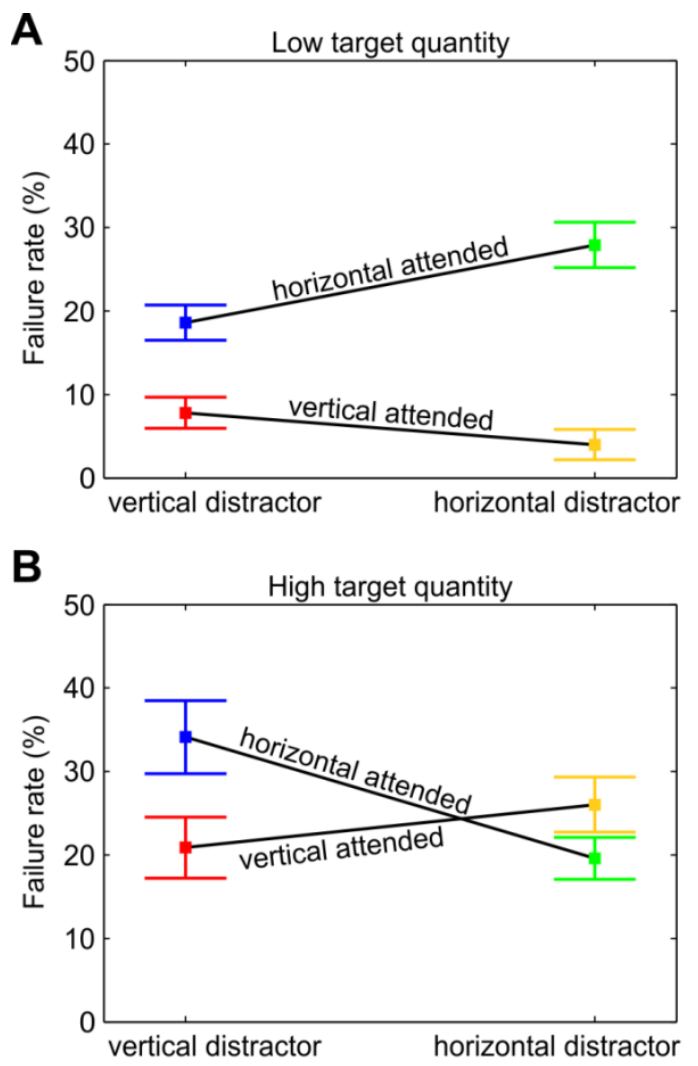

Figure 4. Failure rate. For each of the 4 attentional conditions (vertical/ horizontal attended $x$ vertical / horizontal distractor), the average failure rate (mean \pm repeated measures standard error) is depicted, separately for blocks where less than 5 (low target quantity, panel A) / more than 4 (high target quantity, panel B) target orientations were presented. 
Table 1. ROI information. For the 4 defined ROIs, the size in voxels (mean \pm standard error) before peaking as well as the percent BOLD signal change (mean \pm standard error) within the final $\mathrm{ROI}$ in the respective localizer map is stated.

\begin{tabular}{lll} 
ROI & Size before peaking (in voxels) & $\%$ BOLD signal change \\
\hline Left SI, D4 & $2770 \pm 632$ & $1.5 \pm 0.1$ (D4-map) \\
Left SI, D2 & $4555 \pm 494$ & $2.2 \pm 0.2$ (D2-map) \\
Left SII & $2770 \pm 623$ & $1.4 \pm 0.1$ (D1-to-D5 conjunction map) \\
Right SII & $1066 \pm 405$ & $0.9 \pm 0.1$ (D1-to-D5 conjunction map)
\end{tabular}

Table 2. Failure rate per attentional condition. The failure rate (mean \pm repeated-measures standard error (SEM)) is listed for each attentional condition and target quantity.

\begin{tabular}{lllrll} 
Target quantity & Attended (D4) orientation & Distractor (D2) orientation & mean & \pm & SEM \\
\hline low & vertical & vertical & 7.8 & \pm & 1.8 \\
low & vertical & horizontal & 4.0 & \pm & 1.2 \\
low & horizontal & vertical & 18.6 & \pm & 3.5 \\
low & horizontal & horizontal & 27.9 & \pm & 4.4 \\
high & vertical & vertical & 20.9 & \pm & 4.9 \\
high & vertical & horizontal & 26.0 & \pm & 5.1 \\
high & horizontal & vertical & 34.1 & \pm & 6.7 \\
high & horizontal & horizontal & 19.6 & \pm & 3.8 \\
& & & & \\
\hline
\end{tabular}

Table 3. Failure rates: Statistical evaluation. The repeated-measures ANOVA results are listed, including F-value and $p$-value for each main effect and the interaction as well as $t$-values and p-values for the post-hoc tests. (A) Three-way RM ANOVA. (B) Two-way RM ANOVA for low target quantity. (C) Two-way RM ANOVA for high target quantity.

\begin{tabular}{|c|c|c|}
\hline A Three-way RM ANOVA $\left({ }^{*} p \leq 0.05\right)$ & $F(1,12)$ & p-value \\
\hline Target quantity & 6.9 & $0.022^{*}$ \\
\hline Attended orientation & 28.9 & $<0.001^{*}$ \\
\hline Distractor orientation & $<1$ & 0.638 \\
\hline Target quantity $\mathrm{x}$ Attended orientation & 3.5 & 0.087 \\
\hline Target quantity $\mathrm{x}$ Distractor orientation & 3.2 & 0.099 \\
\hline Attended orientation $\times$ Distractor orientation & $<1$ & 0.466 \\
\hline Target quantity $\mathrm{x}$ Attended orientation $\mathrm{x}$ Distractor orientation & 11.4 & $0.005^{*}$ \\
\hline B Two-way RM ANOVA, low target quantity ${ }^{* *} p \leq 0.025$ ) & $F(1,12)$ & p-value \\
\hline Attended orientation & 27.3 & $<0.001^{* *}$ \\
\hline Distractor orientation & 3.5 & 0.088 \\
\hline Attended orientation $\mathrm{x}$ Distractor orientation & 6.2 & 0.028 \\
\hline Post-hoc tests $\left.{ }^{\star * \star} \mathrm{p} \leq 0.013\right)$ & $t(12)$ & $\mathrm{p}$-value \\
\hline Vertical attended: Vertical versus horizontal distractor & 2.0 & 0.068 \\
\hline Horizontal attended: Vertical versus horizontal distractor & -2.4 & 0.031 \\
\hline C Two-way RM ANOVA, high target quantity $\left({ }^{* *} p \leq 0.025\right)$ & $F(1,12)$ & $\mathrm{p}$-value \\
\hline Attended orientation & $<1$ & 0.506 \\
\hline Distractor orientation & 1.5 & 0.243 \\
\hline Attended orientation $x$ Distractor orientation & 6.7 & $0.024^{* *}$ \\
\hline Post-hoc tests $\left({ }^{\star \star *} p \leq 0.013\right)$ & $t(12)$ & $p$-value \\
\hline Vertical attended: Vertical vers & -1.0 & 0.346 \\
\hline Horizontal attended: Vertical versus horizontal dist & 2.6 & 0.022 \\
\hline
\end{tabular}


For low target quantity (see Table 3B), far higher failure rates were found for horizontal versus vertical attended orientation. Also, a (due to Bonferroni-correction only nearly significant) interaction between "attended orientation" and "distractor orientation" was observed (see Fig. 4A). Breaking this interaction further down, upon attention to the vertical orientation, a trend for higher failure rates was observed if the distractor orientation was also vertical rather than horizontal. A similar trend was seen for attention to the horizontal orientation, where higher failure rates were observed if the distractor orientation was horizontal as well, compared to if it was vertical. Differently phrased, in low-quantity blocks we found trends for higher failure rates in consistent compared to inconsistent blocks for both attended orientations. This interpretation complies with statistics, as a significant interaction in our specific design is equivalent to a main effect of consistency between attended orientation and distractor orientation ${ }^{3}$.

For high target quantity (see Table $3 \mathrm{C}$ ), no main effect but a significant interaction was observed, here reflecting lower failure rates for consistent compared to inconsistent blocks (see Fig. 4B). Breaking down this interaction, only for attention to horizontal a trend for lower failure rates for consistent compared to inconsistent blocks was found.

\section{Attentional analysis: fMRI}

In the ERA analysis, for each ROI (peak voxel along with its neighbors) and attentional condition, a pronounced, similar-shaped (between conditions) BOLD response was observed in the expected time window (Fig. 6A). For the SII ROls, a second increase in BOLD response was observed around the ninth image, hence most probably related to the subjects' foot reaction.

Averaging the response in the defined analysis time-window across time and attentional conditions, the pronounced BOLD activation was statistically confirmed for all ROIs $(\mathrm{t}(12)>3, \mathrm{p}<0.005$ for each ROI). Between ROls, significantly higher activation was observed in "Left SII" compared to "Right SII" $(\mathrm{t}(12)=3.5, \mathrm{p}=0.004)$,

\footnotetext{
${ }^{3}$ As our RM ANOVA design is 2 (horizontal / vertical attended) $\times 2$ (horizontal / vertical distractor), the interaction is basically calculated by comparing the average between vertical attended / vertical distractor and horizontal attended / horizontal distractor with the average of horizontal attended / vertical distractor and vertical attended / horizontal distractor in a paired two-tailed t-test. Hence, a significant interaction in this design is equivalent to a main effect of consistency between attended orientation and distractor orientation.
} 
whereas no significant difference was observed between the two ROls in SI $(t(12)=2.1, p=0.055)$, in contrast to the functional localizer.

Comparing the average BOLD response (within the analysis time-window) between the 4 attentional conditions (Table 4), for the spatially unattended ROI "Left SI, D2" a significantly stronger signal was observed for vertical-attended compared to horizontal-attended blocks (main effect "attended orientation", $F(1,12)=8.6, p=0.012$, see Fig. 5). For all other ROls, no significant differences were observable between conditions (see Fig. $6 \mathrm{~B}$ and Table 5).

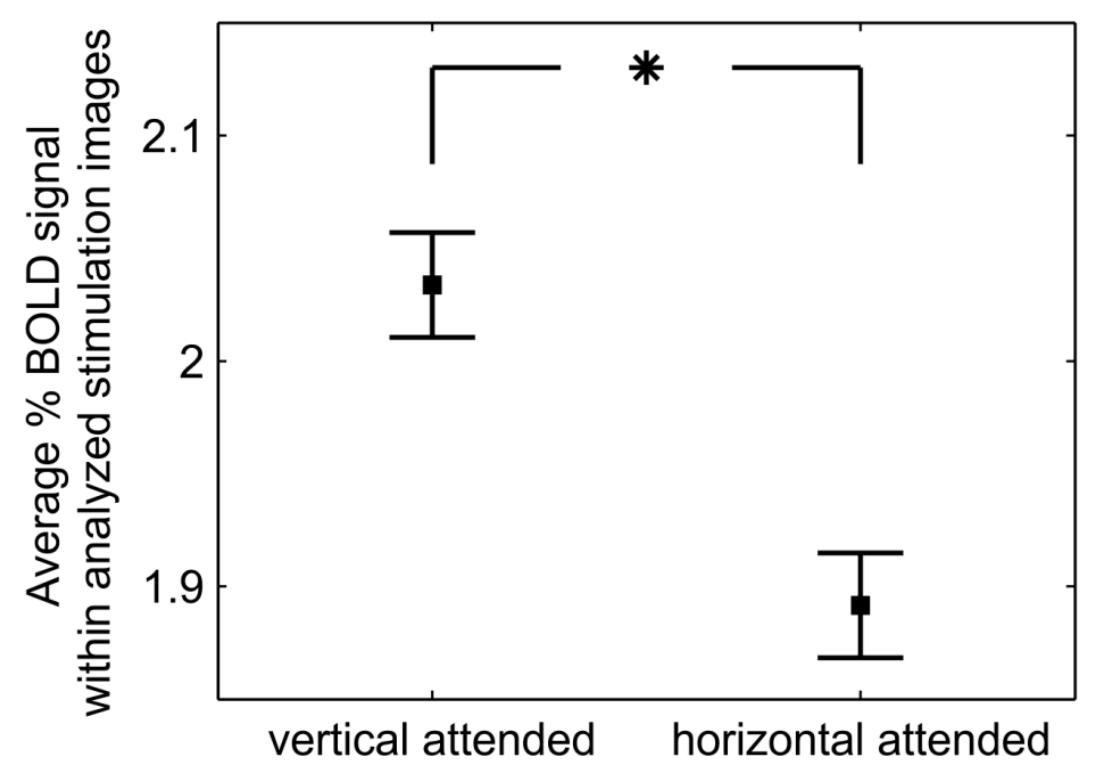

Figure 5. Event-related average analysis of the main effect of "attended orientation" explored in "Left SI, D2", showing higher activation when vertical is attended at D4 compared to when horizontal is attended there. The average BOLD-signal change (mean \pm repeated measures standard error) within the chosen analysis timewindow is shown for vertical- and horizontal-attended blocks, pooling across distractor orientations. ${ }^{*} p \leq 0.013$ 


\subsection{Exploration of feature-based somatosensory modulation of responses to orientations: A human $\mathrm{fMRI}$ study}

Figure 6. Event-related average analysis. (A) The time course of the BOLD-signal change (mean \pm repeated measures standard error) is depicted for each of the 4 attentional conditions (red $=$ vertical attended / vertical distractor, blue $=$ horizontal attended $/$ vertical distractor, yellow $=$ vertical attended $/$ horizontal distractor, green $=$ horizontal attended $/$ horizontal distractor). Baseline (BL), cue (C), stimulation ( $(\mathrm{S})$, and response $(\mathrm{R})$ period are shown. The chosen analysis time-window is framed. (B) The average BOLD-signal change (mean \pm repeated measures standard error) within the chosen analysis time-window is shown for each of the 4 attentional conditions (vertical / horizontal attended $x$ vertical / horizontal distractor).

A

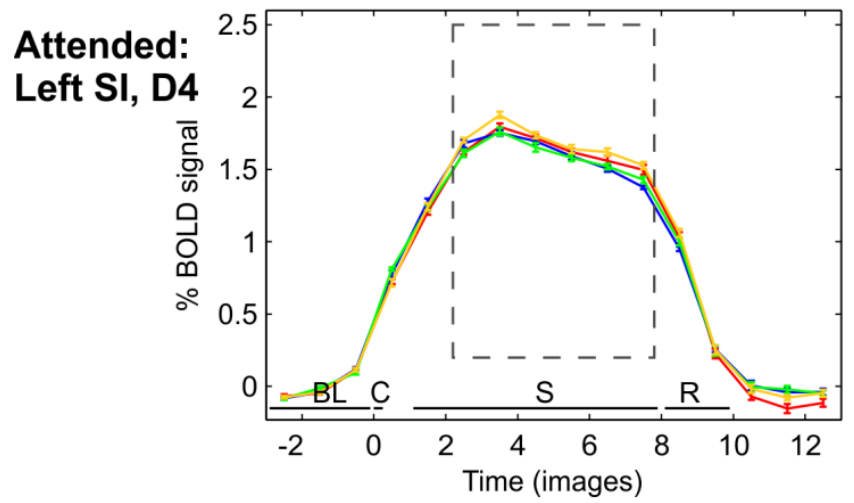

B
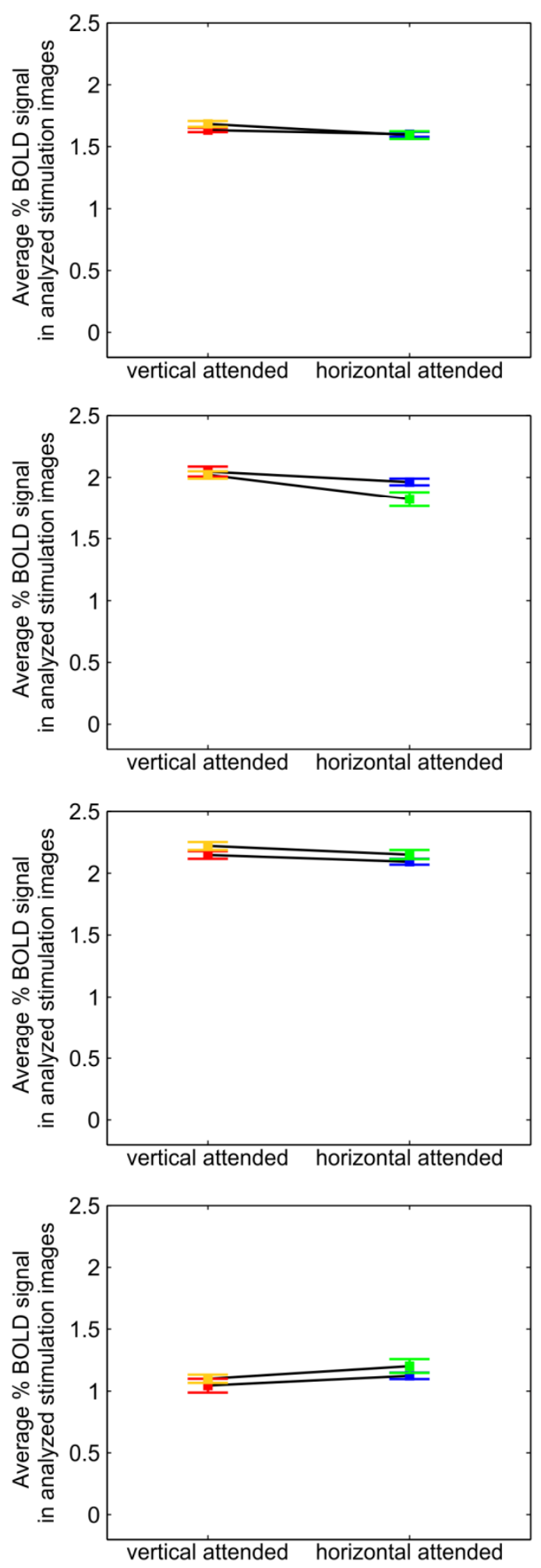
Table 4. BOLD activation per ROI and attentional condition. For each ROI, the average \% BOLD signal change (mean \pm within-ROI repeated-measures standard error) is listed for each attentional condition.

\begin{tabular}{|c|c|c|c|c|}
\hline$\underline{\mathrm{ROI}}$ & Attended (D4) orientation & Distractor (D2) orientation & mean & \pm standard error \\
\hline \multirow[t]{4}{*}{ Left SI, D4 } & vertical & vertical & 1.63 & \pm 0.02 \\
\hline & horizontal & vertical & 1.60 & \pm 0.02 \\
\hline & vertical & horizontal & 1.68 & \pm 0.02 \\
\hline & horizontal & horizontal & 1.59 & \pm 0.03 \\
\hline \multirow[t]{4}{*}{ Left SI, D2 } & vertical & vertical & 2.05 & \pm 0.04 \\
\hline & horizontal & vertical & 1.96 & \pm 0.03 \\
\hline & vertical & horizontal & 2.02 & \pm 0.03 \\
\hline & horizontal & horizontal & 1.82 & \pm 0.06 \\
\hline \multirow[t]{4}{*}{ Left SII } & vertical & vertical & 2.15 & \pm 0.03 \\
\hline & horizontal & vertical & 2.09 & \pm 0.03 \\
\hline & vertical & horizontal & 2.22 & \pm 0.03 \\
\hline & horizontal & horizontal & 2.15 & \pm 0.04 \\
\hline \multirow[t]{4}{*}{ Right SII } & vertical & vertical & 1.04 & \pm 0.06 \\
\hline & horizontal & vertical & 1.12 & \pm 0.03 \\
\hline & vertical & horizontal & 1.10 & \pm 0.03 \\
\hline & horizontal & horizontal & 1.20 & \pm 0.06 \\
\hline
\end{tabular}

Table 5. BOLD activation per ROI: Statistical evaluation. For each ROI, the repeated-measures ANOVA results are listed, including F-value and $p$-value for each main effect and the interaction. ${ }^{*} p \leq 0.013$

\begin{tabular}{lrr} 
Left SI, D4 & $\mathrm{F}(1,12)$ & $\mathrm{p}$-value \\
\hline Attended orientation & 3.3 & 0.095 \\
Distractor orientation & $<1$ & 0.363 \\
Attended orientation x Distractor orientation & 1.1 & 0.305 \\
Left SI, D2 & $\mathrm{F}(1,12)$ & $\mathrm{p}$-value \\
\hline Attended orientation & 8.6 & $0.012^{*}$ \\
Distractor orientation & 2.8 & 0.122 \\
Attended orientation x Distractor orientation & 1.5 & 0.240 \\
Left SII & $\mathrm{F}(1,12)$ & $\mathrm{p}$-value \\
\hline Attended orientation & 2.0 & 0.184 \\
Distractor orientation & 3.1 & 0.104 \\
Attended orientation x Distractor orientation & $<1$ & 0.814 \\
Right SII & & \\
\hline Attended orientation & $\mathrm{F}(1,12)$ & $\mathrm{p}$-value \\
Distractor orientation & 1.6 & 0.234 \\
Attended orientation x Distractor orientation & 2.3 & 0.151 \\
& $<1$ & 0.763
\end{tabular}




\section{Discussion}

In the present study, cortical correlates of tactile global feature-based attention were explored, using a paradigm in which subjects had to attend to a specific orientation at one location (where random orientations were presented) while being stimulated with the same (consistent) or with the perpendicular orientation at another location. On the behavioral level, a modulation of the failure rate could be observed for consistent versus inconsistent blocks, indicating that subjects allocated their feature-based attention to the to-be-attended feature. However, the fMRI data did not allow for localization of an effect of global feature-based attention.

\section{ROIs, digit somatotopy, and digit anisotropy}

The developed ROI-selection process proved to be a successful method with the advantage of automaticity and hence objectivity. This was concluded, as the 5 digit SI ROls showed the expected lateral-to-medial, anterior-to-posterior, and inferior-to-superior succession from thumb to little finger (see Suppl. Fig. 2, panel A-C). Further indications were the pronounced BOLD responses seen in all attentional ROls during attentional runs.

A significantly stronger signal was observed in the D2 ROI upon D2 stimulation compared to the D4 ROI upon D4 stimulation in the functional localizer. Although not statistically assessed here (due to lacking relevance for the question of interest of the current study), the signal observed in the localizer generally seemed to decrease from D1 to D5 stimulation (each assessed in the respective ROI, see Suppl. Fig. 2D). This finding has to our knowledge not been reported before and will be subject of an upcoming manuscript. It is in agreement with psychophysical reports of increasing grating-orientation discriminationthresholds from D2 to D4 (Vega-Bermudez et al., 2001), potentially related to a more relevant role of D2 for everyday tactile exploration (Vega-Bermudez et al., 2001; Schweisfurth et al., 2011; Schweisfurth et al., submitted).

\section{Spatial attention}

Although spatial attention could not explicitly be explored with our design, we found indications that subjects indeed focused onto the ring finger. The first and 
strongest hint is the high performance of subjects in the psychophysical task. On the cortical level, the difference in activation (percent BOLD signal change) between "Left SI, D2" and "Left SI, D4" was much lower (and not any more significant) in the attentional measurements compared to the localizer session, due to an increase in D4-ROI activation along with a decrease in D2-ROI activation, probably upon spatial attention to D4 (compare Table 1 and 4). However, this comparison between localizer-run and attentional-run activation levels should be considered with caution, as the designs (sequentially-blocked versus blocked, one-digit versus multiple-digit stimulation, $32 \mathrm{~Hz}$ versus $10 \mathrm{~Hz}$ ) strongly differed between these measurements.

\section{Feature-based attention}

While the psychophysical measures depended on the consistency between attended feature and distractor feature, so far we could not detect an fMRI effect of feature-based attention in the sense suggested by the feature-similarity gain model (Treue and Martinez-Trujillo, 1999).

Psychophysics. Behavioral data revealed that the failure rate depended on the consistency between attended feature and distractor feature (see Fig. 4), which could be seen both in those blocks where less than 5 target orientations were shown (low target-quantity blocks) and in those where more than 4 target orientations were shown (high target-quantity blocks). In low target-quantity blocks, the failure rate can roughly be interpreted as false-alarm rate, as a wrong button press indicates that the subject counted one or more targets than actually presented. In high target-quantity blocks, in contrast, the failure rate can roughly be understood as miss rate, as a wrong button press points to the subject having missed at least one target. In low target-quantity blocks, subjects presented with a trend for higher false-alarm rates during consistent blocks (where the orientations attended at D4 and presented at D2 were the same) compared to inconsistent blocks; this most probably resulted from occasional counting of an orientation-consistent D2-distractor as target, despite its presence at the irrelevant finger. Along the same lines, the miss rate was lower for consistent compared to inconsistent blocks for high target-quantity blocks; most probably, subjects occasionally counted orientation-consistent D2-distractors as targets and thereby were more likely to correctly judge that more than 4 targets had been presented. This effect does not point to an effect of feature-based attention in 
the sense suggested by the feature-similarity gain model; it rather shows that subjects were not able to entirely focus their attention onto the to-be-attended location.

$f M R I$. The expected cortical effects of global feature-based attention could not be detected. Our expectations resulted from similarly designed visual fMRI (Saenz et al., 2002) and tactile ERP (Forster and Eimer, 2004) experiments. In the cited visual study, subjects had to spatially attend to a spot where two opposite motion directions were simultaneously presented; more precisely, they should focus on just one of the motion directions and detect related speed changes. At the same time, at a distant distractor location subjects were stimulated by just one of these two motion directions. A higher fMRI signal was found in the cortical representations (in different hierarchical areas) of the distractor location, if the distantly attended and the locally presented motion directions were the same compared to if they differed, pointing to a global effect of feature-based attention present already in early visual areas. Similarly, the tactile feature-based attention study (Forster and Eimer, 2004) found that attention directed to one out of two intensities led to an enhancement of a negative EEG component (N140) followed by a sustained negativity for intensitymatched compared to intensity-unmatched stimuli. Similarly, attention to one out of two stimulation frequencies resulted in early enhanced negativity (80 - 140 ms after stimulus onset, only observed for attending to the lower frequency) for frequencymatched compared to frequency-unmatched stimuli, again followed by a sustained negativity. Most importantly, these effects were observed not only at the attended and behaviorally-relevant location but also at an unattended location at the other hand, giving a first piece of evidence for the existence of global feature-based attention in touch. Having these previous inter- and intra-modal findings in mind, we expected to find analogous response patterns (sketched in Suppl. Fig. S3, ignoring potential processing differences between horizontal and vertical orientation that might influence the response patterns): For the distractor location ("Left SI, D2"), an interaction was expected (see Suppl. Fig. S3B), due to a stronger signal for distantly attending to the same compared to distantly attending to the perpendicular orientation, for each distractor orientation. However, the observed pattern did not depend on the attended-orientation / distractor-orientation consistency (Fig. 5 and 6, Table 5). At the attended location (see Suppl. Fig. S3A), we did not expect a change 
due to feature-based attention, which indeed was in agreement with our observations (Fig. 5, Table 5). For the second somatosensory cortex, we hypothesized a higher signal for consistent compared to inconsistent blocks (see Suppl. Fig. S3, panel C and D), similar as for the distractor location; this pattern could not be confirmed (Fig. 5, Table 5). If present, feature-based modulations are also visible by calculating the attentional modulation similar as done in the visual study by Saenz and colleagues (2002); in our case, it did not reveal positive signal modulation (see Suppl. Fig. S4). At the current stage, we do not have a final explanation why we did not find effects of feature-based attention in the described classical sense in SI or SII. Looking at this fMRI study alone, one could argue that attention to orientations for some reason does not lead to these feature-based attentional effects in early somatosensory areas, as also no such effects could be observed for the higher frequency in the Forster and Eimer study (2004). However, a previous purely psychophysical study by our own group (Schweisfurth et al., in preparation) strongly points to the existence of global feature-based attentional effects at the behavioral level for exactly the features used here. Apart from the stimulus dimension, there is another important difference between our present study and the one by Forster and Eimer (2004). In their study, only one feature was presented per trial, either at the attended or unattended location and either with attended or unattended feature. Subjects should only react to targets (which were of $680 \mathrm{~ms}$ duration, compared to $340 \mathrm{~ms}$ pulses in normal trials) at the attended location having the attended feature. Hence, there was no severe pressure to suppress pulses at the other location even when it had the attended feature, as pulses were already presented for $340 \mathrm{~ms}$ when the difference between behaviorally-relevant (correct combination of attended location and feature) and irrelevant targets was determinable. Also, the observed false-alarm rate was very low in comparison to our own previous tactile feature-based reaction-time study $(<1 \%$ versus $>7 \%$ in Schweisfurth et al., in preparation), indicating enough processing time for the presented stimulus. In our case, however, D4 and D2 orientations were simultaneously presented and subjects hence ought to have actively ignored the index finger. Speculatively, such an active ignoring of a location (imaginable as the antipode of spatial attention) might be stronger in consistent compared to inconsistent blocks and hence have a larger suppressive effect, which 

to orientations: A human fMRI study

might compensate for the expected signal-increasing feature-based effect, such that the activation level remains similar between consistent and inconsistent blocks.

\section{Orientation anisotropy}

A lower false-alarm rate and a higher BOLD signal at the ignored finger were observed when subjects attended to the vertical orientation compared to when they attended to the horizontal orientation, suggesting not only an anisotropy between vertical and horizontal orientation but also a distant cortical effect of attention to one orientation compared to another.

Psychophysics. Comparing between attended orientations, a higher performance was found for attention to vertical compared to horizontal for low targetquantity blocks, hence indicating that subjects were less likely to mistake D2-distractors (of any orientation) as targets when attending to the vertical compared to when attending to the horizontal orientation. This suggests an anisotropic processing or perception of orientations in our experiment. We hypothesize that this is due to everyday haptic experiences: While striping across a natural object, at least one of its edges usually simultaneously touches several fingertips (moving relatively to them along the proximal-to-distal axis) with horizontal or oblique orientation; for the vertical orientation, this only happens if the object itself comprises several parallel edges. Hence, it might be better possible to ignore the D2 stimulation in vertical-attended compared to horizontal-attended blocks. Better performance for attention to the vertical compared to the horizontal orientation is also supported by the observation of faster reaction times for vertical compared to horizontal targets in our previous reaction-time study using exactly the same features (Schweisfurth et al., in preparation). The question of orientation anisotropy is still under debate in the psychophysical literature; an overview has been given in our just cited psychophysical paper.

fMRI. In interesting analogy to the psychophysical data, at the distractor location ("Left SI, D2") a significantly stronger signal was observed for attending (at D4) to the vertical compared to the horizontal orientation. This attentional effect suggests different ways of SI processing in our experiment depending on which of the two orientations is attended. A potential but very speculative explanation for the finding is the following: Spatially attending to D4 should suppress the D2 signal 
compared to if D2 was attended (to our knowledge so far not rigorously tested; for related effects, see Discussion section on spatial attention and Eimer and Forster, 2003; Johansen-Berg et al., 2000). Now, whenever the horizontal orientation is attended at D4, it is harder for the subject to ignore D2, as already discussed in the psychophysics paragraph. A cortical compensation mechanism for this difficulty could be to suppress the index finger slightly stronger for horizontal-attended compared to vertical-attended blocks. Obviously, one could also conversely hypothesize that attention to the vertical orientation at D4 increases the D2 signal (compared to attention to horizontal at D4) for certain reasons; however, we currently do not see any neuroscientific reason for this possibility, rendering it less likely to us. Yet, for shedding more light into this issue, more experiments are needed to clarify the SI correlates of spatial attention varying between digits as well as possible crosstalk between digits and possible anisotropy between digits and orientations. One such supplementary pilot study (see Suppl. Fig. S5 for methodological details and preliminary results) has already been run but so far did not yield any significant results or trends that would be suitable for explanation of the effects observed here. Earlier studies on monkey SI found either a trend for less neurons with preferred orientation 'vertical' than with other preferred orientations (DiCarlo and Johnson, 2000) or could not find any tendency for an orientation being overrepresented (Bensmaia et al., 2008a). In analogy to the latter, we also did not observe any signal difference between vertical and horizontal distractors.

\section{Technical considerations}

On the technical side, we could exclude that an error in the programmed MWorks / FSL / BrainVoyager / MATLAB analysis routine was responsible for the findings of the present study, as a control experiment ${ }^{4}$ was run in three subjects as sanity check for the analysis procedure. In that experiment, the 4 conditions (vertical / horizontal attended at D4 combined with same/ different distractor presented at D2) were replaced by strong / weak stimulation to only D2 / only D4,

\footnotetext{
${ }^{4}$ The main reason for running this experiment was that -at that point in time- the analysis routine revealed highly significant but highly unexpected results for all ROls. As the sanity check showed that the individual-subject analysis worked well, we investigated the BrainVoyager 2.4 and 2.6 eventrelated average (ERA) tools used for across-subjects analysis and found an inconsistency there, which finally turned out to be responsible for the unexpected previous results.
} 
with the cue being substituted by a weak pulse to both digits. Thus, only the specific stimulation was changed, with the overall design staying the same. Hence, we could use exactly the same analysis routine as for the attentional runs, allowing for critical testing of the latter. With these modifications, we expected for each SI digit ROI a higher signal if the ROI-defining digit was stimulated. Also, a higher signal should be observable upon strong compared to weak stimulation in the stimulated digit's ROI. If these expectations were met, we could then conclude that the individual-subject analysis routine should be correct. Indeed, the expected cortical responses were mostly met in both "Left SI, D2" and "Left SI, D4" of individual subjects (see Suppl. Fig. 6): A pronounced BOLD activation was observed for strong stimulation of D2 and D4 in the respective digit's ROI, while only weak or no activation was found in the respective weak condition. If the other digit (D2 for "Left SI, D4" and D4 for "Left SI, D2") was weakly or strongly stimulated, much less activation was seen. Hence, it was concluded that the individual-subject analysis was correct. With successful circumvention of an inconsistency (described in the Methods section) in the BrainVoyager-calculated event-related averages (ERA) used for across-subjects analysis, the analysis routine should at the present stage be entirely correct.

A serious statistical argument for not finding an expected result (as in the present study an fMRI correlate of feature-based attention in the classical sense) always is that the number of subjects might have been too low. But we included 13 subjects in our study, which is far more than included in the analogous visual study (3 subjects, Saenz et al., 2002) and in the same range as in the tactile featurebased attention study (12 subjects; Forster and Eimer, 2004). However, the number of averages per subject may have been too low. In our case, the event-related average was calculated from 40 repetitions per condition, whereas the cited visual fMRI and somatosensory ERP studies achieved 72 and 400 repetitions, respectively. To get an impression of whether the number of repetitions might have critically influenced the result, we looked at the two sessions separately (see Suppl. Fig. S7 for an example). While the average size and time course was nicely reproducible in each ROI, we indeed observed small but critical intra-condition differences between sessions, pointing to the fact that 20 repetitions per condition would not have been enough to get reproducible patterns between conditions. However, it is hard to say 
whether our 40 repetitions were already good enough to compensate for random between-block variations.

In the future, the differences between attentional conditions should not only be explored for $\mathrm{SI}$ in its entirety but separately for BAs 3b, 1, and 2. At the moment, the registration between the Jülich atlas and the FSL standard brain is not good enough for this purpose; however, it will be improved in the next release of the FSL Anatomy Toolbox (personal communication with Simon Eickhoff). Then, area-specific analysis of the present data should be possible and will be carried out.

\section{Conclusion and outlook}

In summary, this paper reports the first fMRI study (and one of the first studies in general) devoted to the exploration of tactile feature-based attention. So far, no cortical effect of feature-based attention could be detected in the primary or secondary somatosensory cortices. Psychophysical and cortical alterations were observed depending on which orientation was attended at D4, reflecting a global, orientation-dependent attentional effect, pointing to an anisotropy in the processing or perception of orientations. The present study has raised several important issues that should be addressed by further research in the field of tactile spatial attention, tactile orientation anisotropy, and most importantly tactile feature-based attention. 
2.4 Exploration of feature-based somatosensory modulation of responses to orientations: A human fMRI study

\section{Acknowledgements}

The current work was supported by the International Max Planck Research School MSc/PhD/MD-PhD Neuroscience Program (to M.A.S.) of the Göttingen Graduate School of Neurosciences and Molecular Biosciences (GGNB) in Göttingen, Germany, and the graduate school Neurosenses (to M.A.S.) in Göttingen and Oldenburg, Germany. Further on, we thank Dr. Tibor Auer for help with respect to the FSL-ROI analysis and Benjamin Beeck for suggesting the sanity-check experiment. 


\section{Supplementary Figures}

Supplementary Figure S1. Event-related average analysis taking into account ROIs before peaking. (A) The time course of the BOLD-signal change (mean \pm repeated measures standard error) is depicted for each of the 4 attentional conditions ( $r e d=$ vertical attended / vertical distractor, blue $=$ horizontal attended / vertical distractor, yellow $=$ vertical attended / horizontal distractor, green $=$ horizontal attended / horizontal distractor). Baseline (BL), cue (C), stimulation, and response (R) period are shown. The chosen analysis time-window is framed. (B) The average BOLDsignal change (mean \pm repeated measures standard error) within the chosen analysis time-window is shown for each of the 4 attentional conditions (vertical / horizontal attended $\mathrm{x}$ vertical / horizontal distractor). No differences could be observed between conditions for any non-peaked ROI by repeated-measures ANOVA (all p-values $>0.1$ )

A

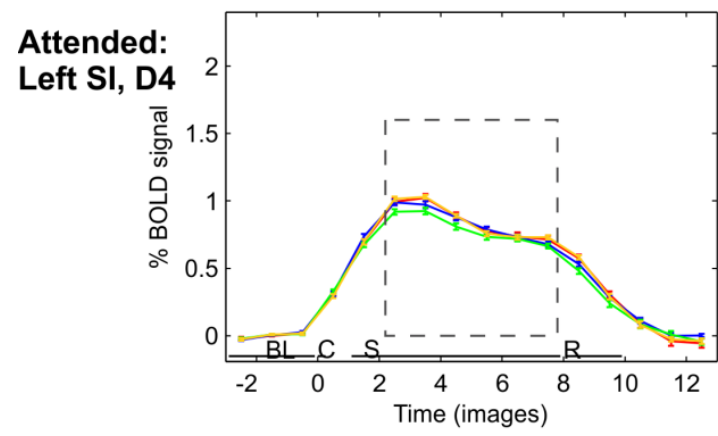

Distractor:

Left SI, D2

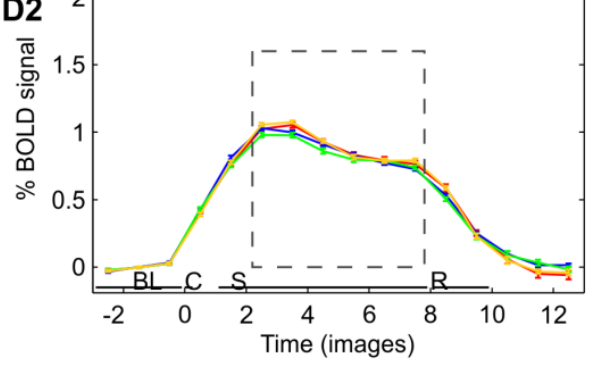

Left SII
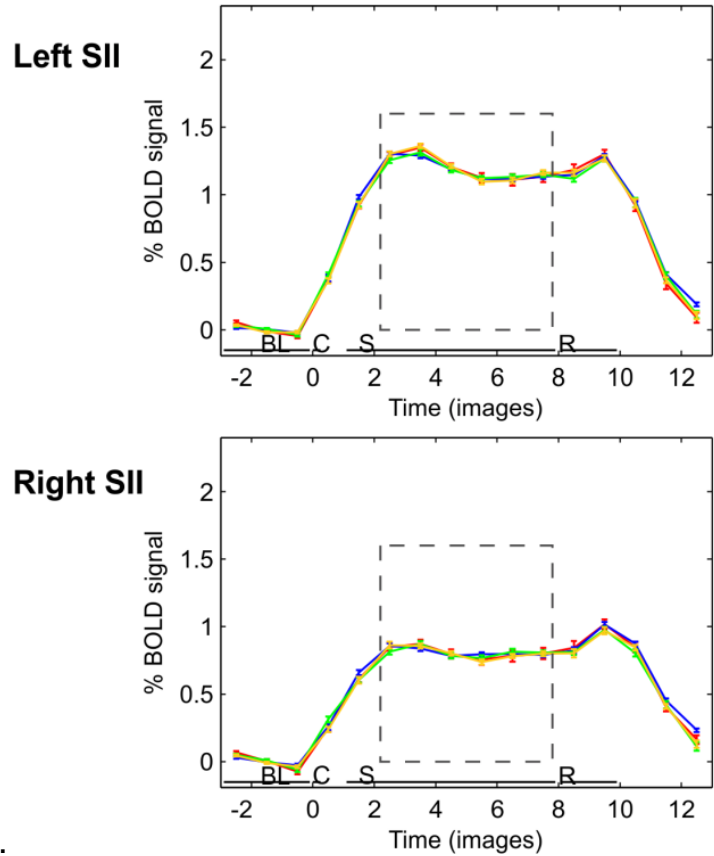

B
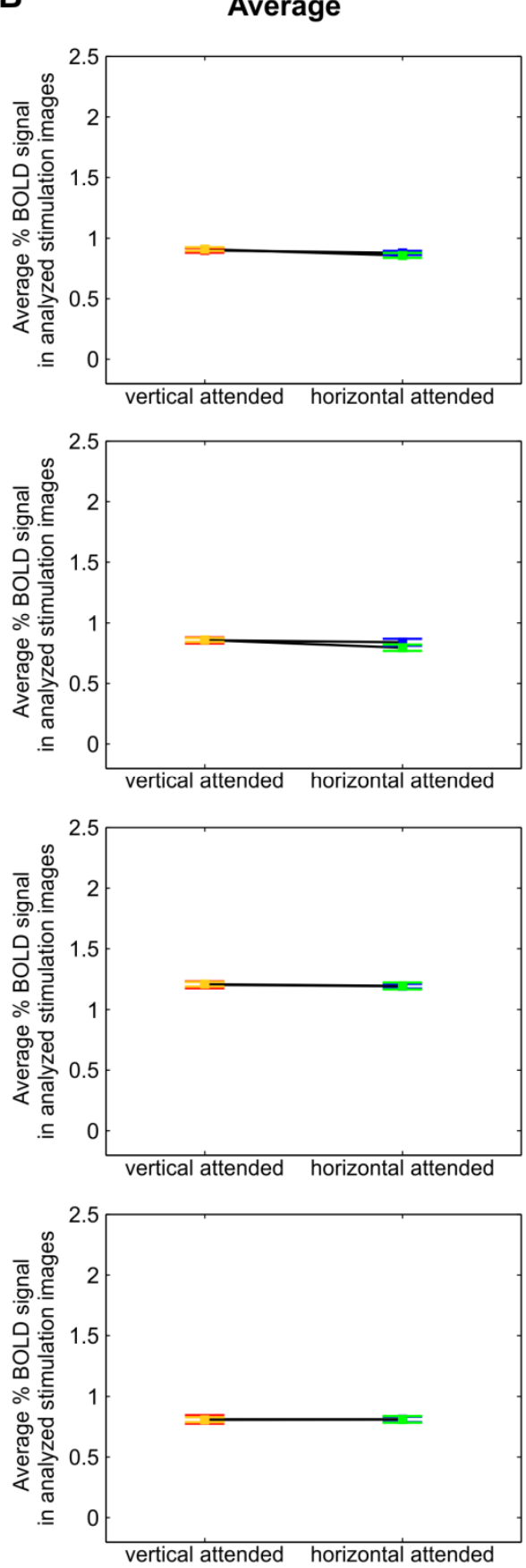


\subsection{Exploration of feature-based somatosensory modulation of responses}

to orientations: A human fMRI study

Supplementary Figure S2. SI somatotopy observed in localizer. Panel A-C: For each digit (thumb $=1$ to little finger $=5$ ), the average peak-voxel ACPC coordinate (mean \pm repeated-measures standard error) is stated for each axis. (A) $x$-coordinates, running from medial (low values) to lateral (high values). (B) y-coordinates, running from anterior (low values) to posterior (high values). (C) z-coordinates, running from superior (low values) to inferior (high values). (D) The average \% BOLD signal (mean \pm repeatedmeasures standard error) is presented for each digit, assessed in the respective digit's ROI.
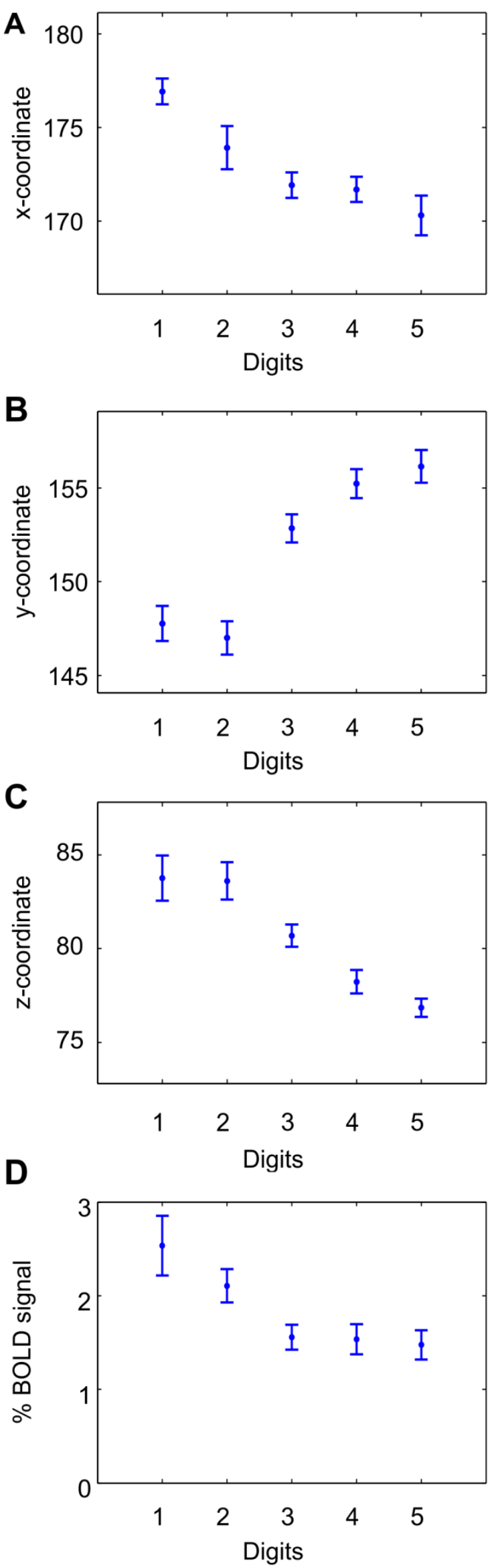
Supplementary Figure S3. Feature-based expectation scheme. This figure shows what we expected to find, if global feature-based attention in the classical sense exists within the primary or secondary somatosensory cortex. For the distractor location (panel B, "Left SI, D2") as well as for the secondary areas (panel $C$ and D), a stronger signal should be observed for the consistent compared to the inconsistent conditions, detectable by a significant interaction between attended orientation at D4 and presented orientation at D2 (see footnote 3). For the attended location (panel A, "Left SI, D4"), we did not except significant differences between conditions, as the presented feature randomly varied here.
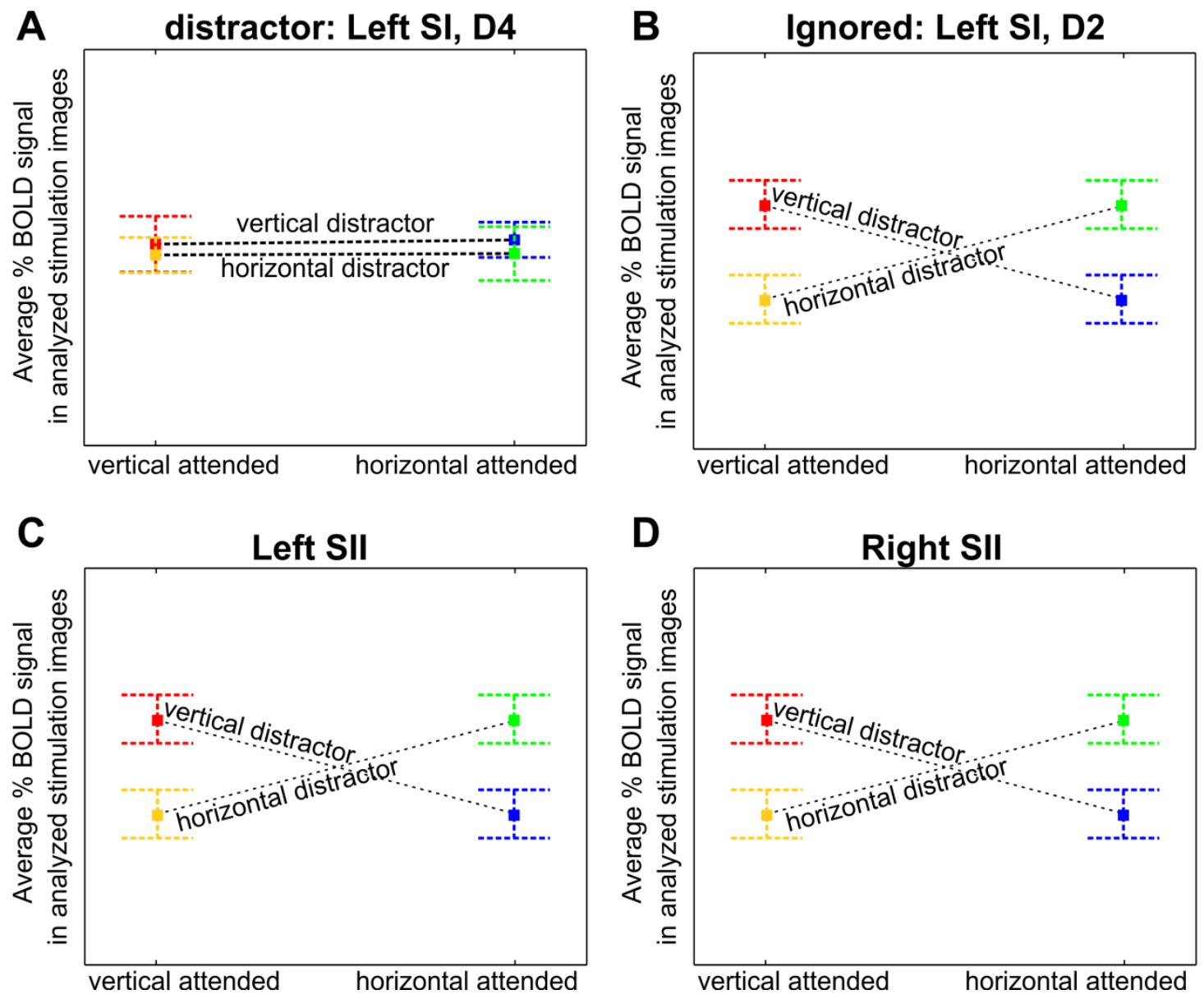


\subsection{Exploration of feature-based somatosensory modulation of responses to orientations: A human fMRI study}

Supplementary Figure S4. Feature-based attentional modulation. For better comparison of our study with the visual feature-based fMRI study by Saenz et al. (2002), we calculated the relative attentional modulation in each ROI. Therefore, the time courses for vertical and horizontal distractors were averaged, separately for consistent and inconsistent conditions. Then, the attentional modulation was based on the average percent signal change in the chosen analysis time-window and calculated by Attentional modulation $=100 \% \times \frac{\text { consistent }- \text { inconsistent }}{\text { consistent }}$, such that $0 \%$ would reflect no attentional modulation upon feature-based attention and 100\% an attentional modulation as strong as complete removing of the stimulus. In our data, no significant modulation could be observed, as seen in this figure where the attentional modulation (mean \pm standard error) is depicted for each ROI.

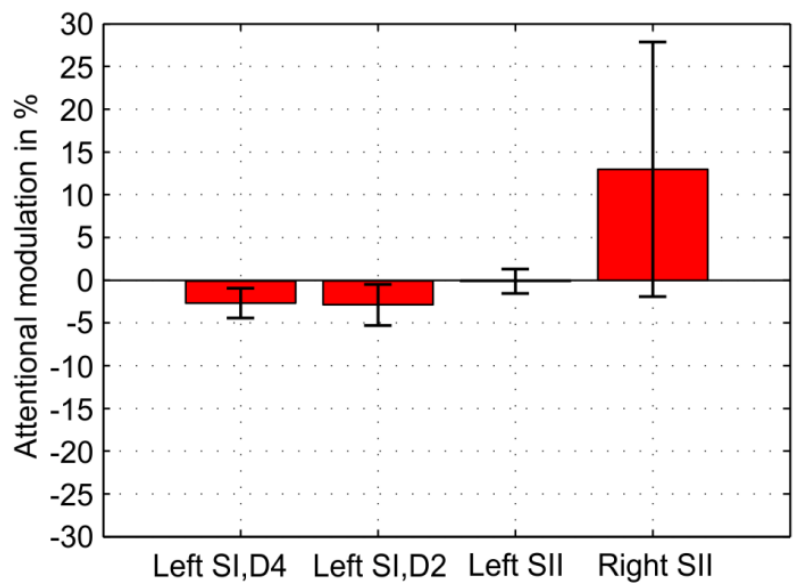


Supplementary Figure S5. Crosstalk experiment - methods and preliminary results. To explore the crosstalk between the digits, a supplementary experiment was run in 4 subjects (4 runs per subject). Within a similar paradigm as for this study's attentional runs, in each block subjects received simultaneous stimulation to D2 and D4. From block to block, they were asked to alternate their spatial attention between the two fingers. At the attended finger, a cross (generated by raising all pins for the vertical and horizontal orientation) was presented and at random time points substituted by one-dot targets, which were to be counted. The unattended finger received either vertical or horizontal stimulation throughout the block. That design allows to test whether signal differences in the unattended finger's ROI are observable between vertical and horizontal presentation and whether such differences are also present for the attended finger, i.e. whether come kind of orientation crosstalk can be shown between fingers. Due to the low sample size, only first observations can be reported here. For "Left SI, D2", we found no differences between the attend-in / attend-out and between the vertical / horizontal conditions. For "Left SI, D4", again no crosstalk effect was observable; however, there was a trend for lower signal for presentation of the vertical compared to the horizontal orientation at the unattended ring ringer. For none of the ROls, clear effects of spatial attention were visible. As no clear trends could be deduced from this supplementary experiment, it could not be used for explanation of the main study's results.

Time course

Left SI, D4

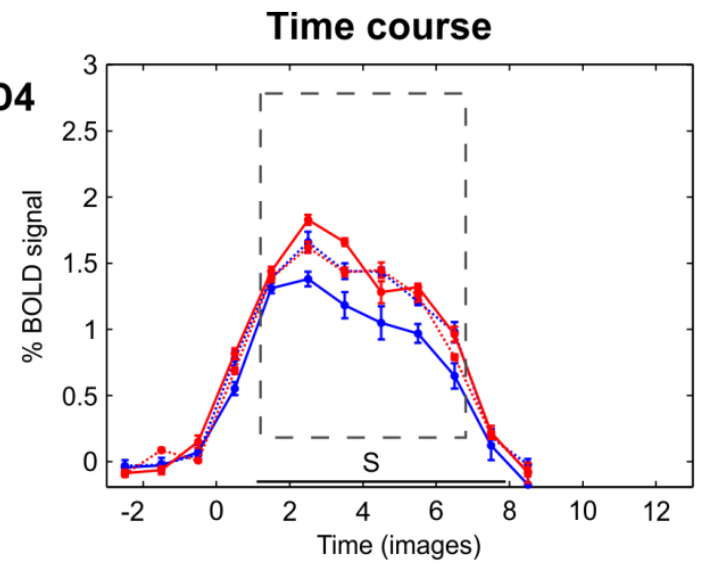

Left SI, D2

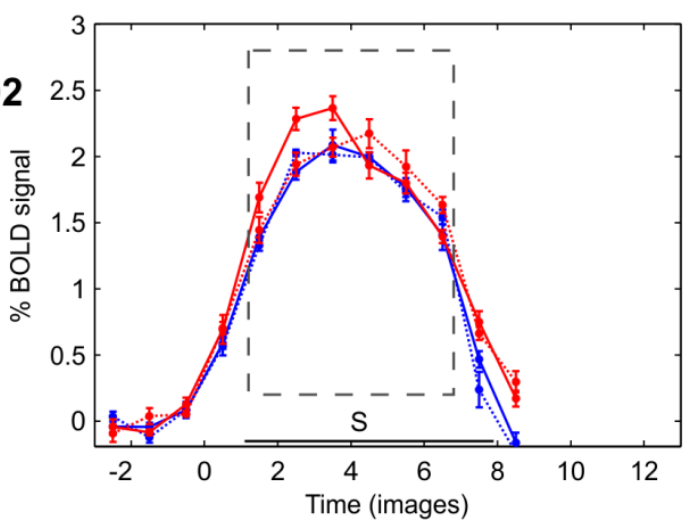

Average
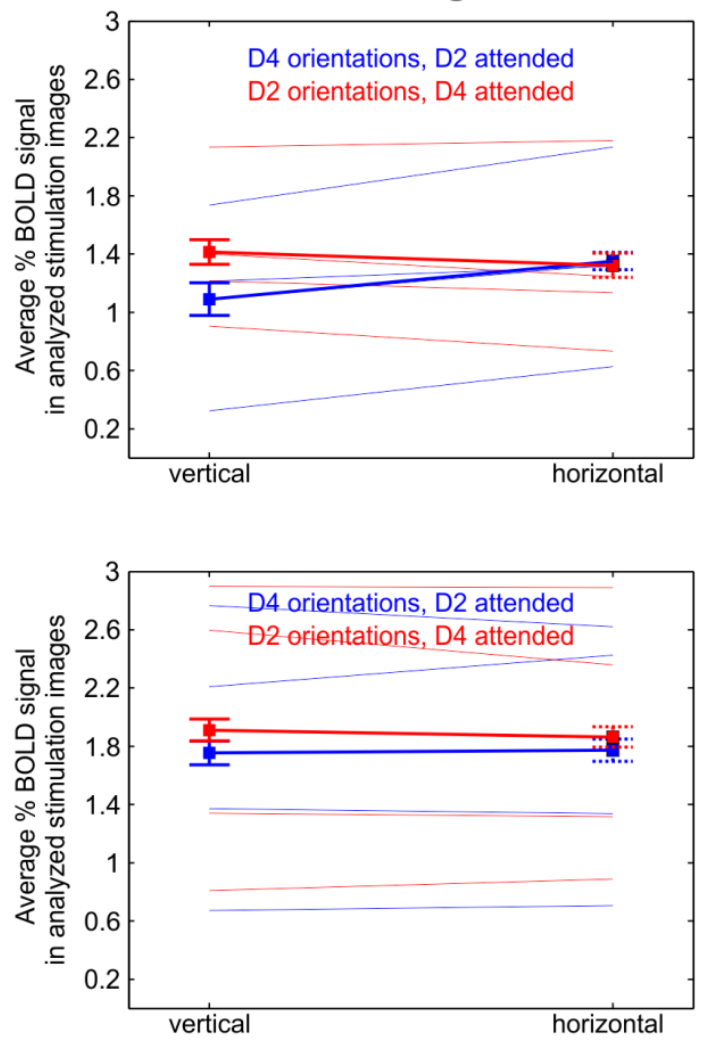


\subsection{Exploration of feature-based somatosensory modulation of responses}

to orientations: A human fMRI study

Supplementary Figure S6. Sanity-check experiment. For three subjects (each presented in one row), the average percent BOLD signal in the analysis time-window across two runs (for subject V2 only one run) is plotted for each stimulation condition (strong / weak stimulation of D2 / D4). As strong signal, all 17 pins (for $\mathrm{V} 1$, only the 5 vertical-generating pins) were raised to $1.5 \mathrm{~mm}$ (measured without pressure from above), while for the weak signal only the central pin was raised to $0.75 \mathrm{~mm}$ (without pressure).

Left SI, D4

V1

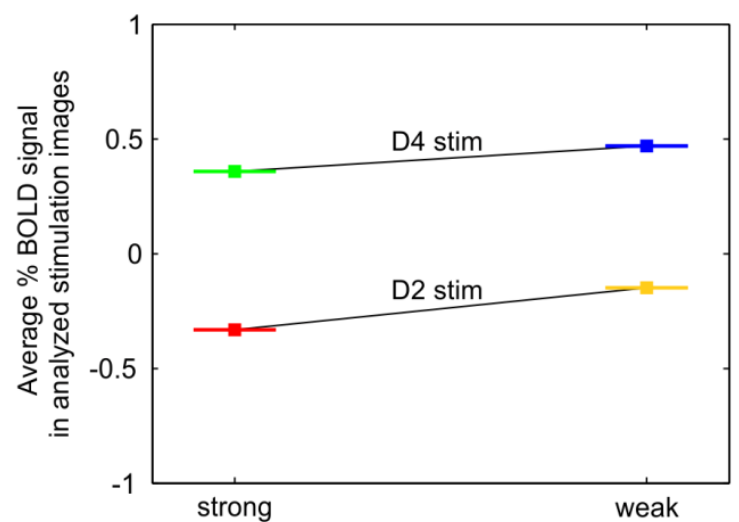

V2

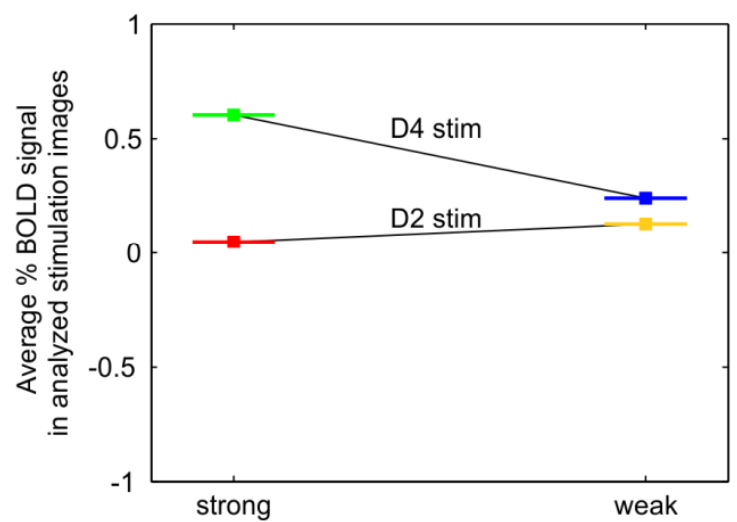

V8

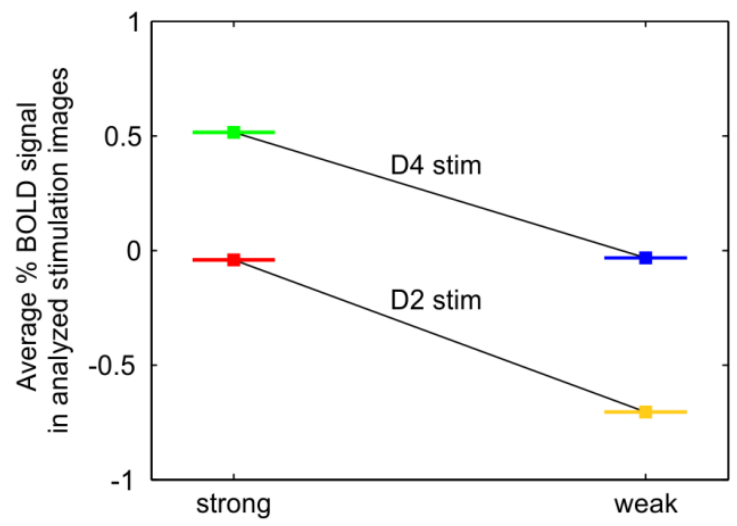

Left SI, D2
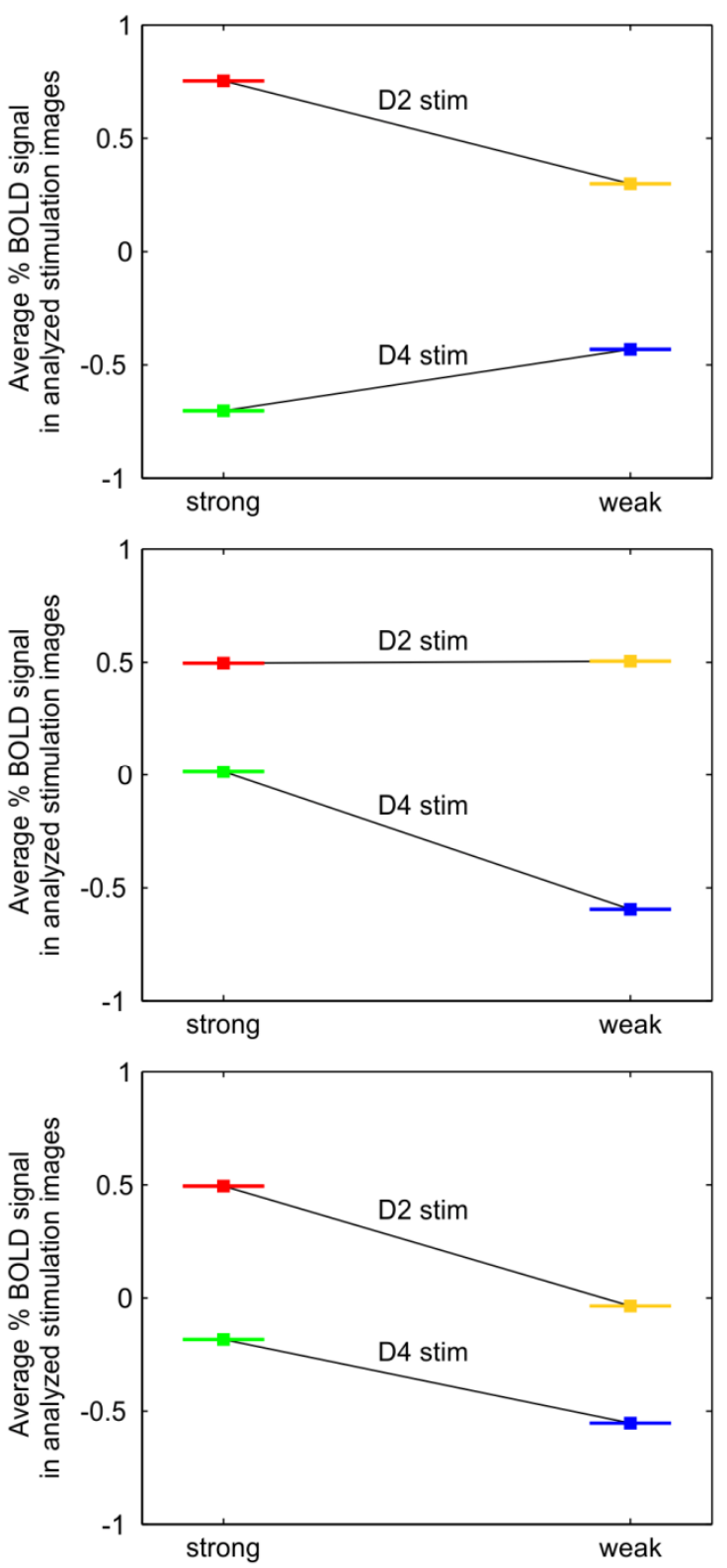
Supplementary Figure S7. Inter-session variations of "Left SI, D2". Analogous to Fig. 6, the time course of the BOLD-signal change (upper row) as well as the average BOLD-signal change (lower row) are depicted for each of the 4 attentional conditions (red = vertical attended / vertical distractor, blue $=$ horizontal attended / vertical distractor, yellow $=$ vertical attended / horizontal distractor, green $=$ horizontal attended $/$ horizontal distractor). The left and right columns illustrate the results for Session 1 / Session 2. While the general activation level is very similar across sessions, the average analysis (with RM-ANOVA results stated within the images) points to differing trends (being far from significance, though, as only $p$-values $\leq 0.006$ would be significant due to Bonferroni correction (4 ROls $\times 2$ sessions). These results indicate that combining at least two sessions is required for reliable analysis and that even more sessions per subjects might have been preferable.

Time course session 1

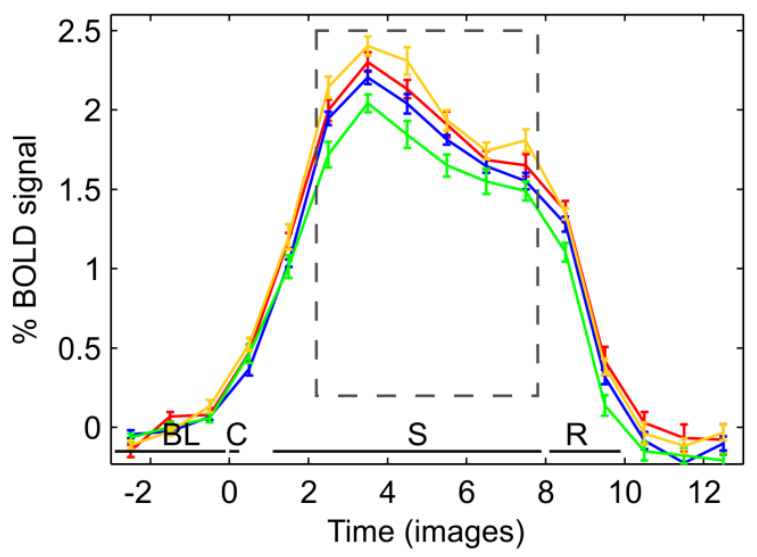

Average session 1

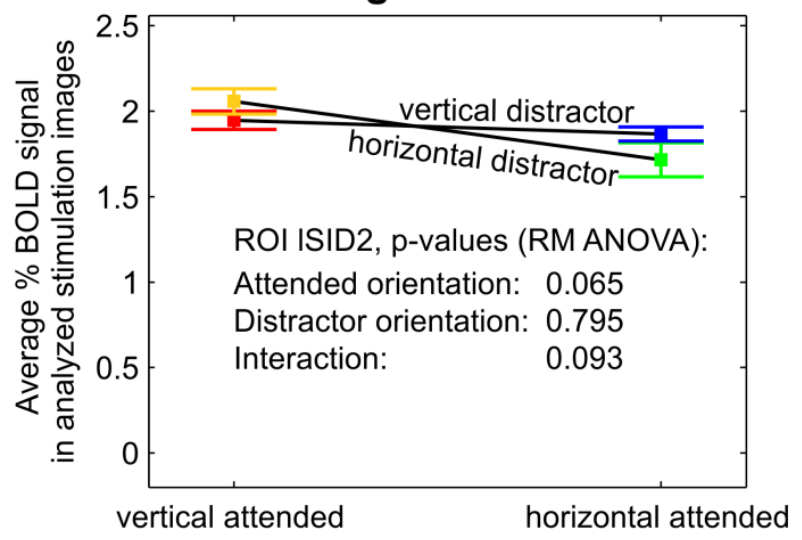

Time course session 2

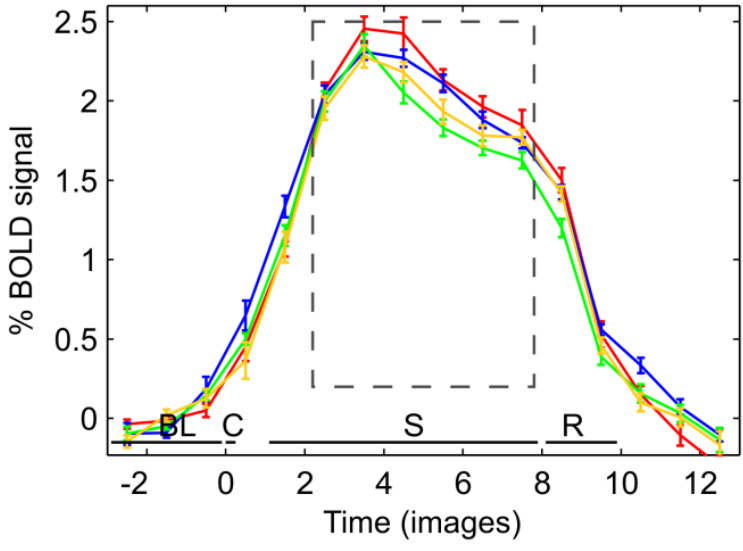

Average session 2

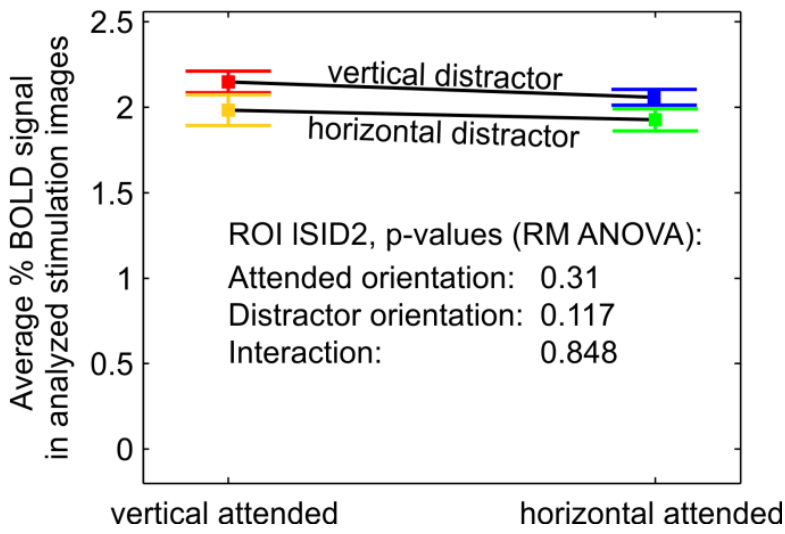




\subsection{Exploration of feature-based somatosensory modulation of responses}

to orientations: A human fMRI study

\section{References}

Bensmaia SJ, Denchev PV, Dammann III JF, Craig JC, Hsiao SS (2008a) The representation of stimulus orientation in the early stages of somatosensory processing. Journal of Neuroscience 28:776-786.

Bensmaia SJ, Hsiao SS, Denchev PV, Killebrew JH, Craig JC (2008b) The tactile perception of stimulus orientation. Somatosensory and Motor Research 25: 49-59.

Burton H, Abend NS, MacLeod AMK, Sinclair RJ, Snyder AZ, Raichle ME (1999) Tactile attention tasks enhance activation in somatosensory regions of parietal cortex: A positron emission tomography study. Cerebral Cortex 9: 662-674.

Burton H, Sinclair RJ, McLaren DG (2008) Cortical network for vibrotactile attention: A fMRI study. Human Brain Mapping 29: 207-221.

Castelo-Branco M, Kozak LR, Formisano E, Teixeira J, Xavier J, Goebel R (2009) The type of featural attention differentially modulates hMT+ responses to illusory motion after effects. Journal of Neurophysiology 102: 3016-3025.

DiCarlo JJ, Johnson KO (2000) Spatial and temporal structure of receptive fields in primate somatosensory area $3 \mathrm{~b}$ : effects of stimulus scanning direction and orientation. The Journal of Neuroscience 20: 495-510.

Eickhoff SB, Schleicher A, Zilles K, Amunts K (2006a) The Human Parietal Operculum. I. Cytoarchitectonic Mapping of Subdivisions. Cerebral Cortex 16: 254-267.

Eickhoff SB, Amunts K, Mohlberg H, Zilles K (2006b) The Human Parietal Operculum. II. Stereotaxic Maps and Correlation with Functional Imaging Results. Cerebral Cortex 16:268-279.

Eimer M, Forster B (2003) The spatial distribution of attentional selectivity in touch: evidence from somatosensory ERP components. Clinical Neurophysiology 114: 1298-1306.

Fitzgerald PJ, Lane JW, Thakur PH, Hsiao SS (2006) Receptive field properties of the macaque second somatosensory cortex: Representation of orientation on different finger pads. Journal of Neuroscience 26: 6473-6484.

Forster B, Eimer M (2004) The attentional selection of spatial and non-spatial attributes in touch: ERP evidence for parallel and independent processes. Biological Psychology 66: 1-20.

Friston KJ, Fletcher P, Josephs O, Holmes A, Rugg MD, Turner R (1998) Event-related fMRI: Characterizing differential responses. Neurolmage 7: 30-40.

Galazky I, Schütze H, Noesselt T, Hopf J-M, Heinze H-J, Schoenfeld MA (2009) Attention to somatosensory events is directly linked to the preparation for action. Journal of the Neurological Sciences 279:93-98.

Gelnar PA, Krauss BR, Szeverenyi NM, Apkarian AV (1998) Fingertip representation in the human somatosensory cortex: an fMRI study. Neurolmage 7:261-283.

Goebel R, Esposito F, Formisano E (2006) Analysis of functional image analysis contest (FIAC) data with brainvoyager QX: From single-subject to cortically aligned group general linear model analysis and self-organizing group independent component analysis. Human Brain Mapping 27: 392-401.

Hämäläinen H, Hiltunen J, Titievskaja I (2000) fMRI activations of SI and SII cortices during tactile stimulation depend on attention. NeuroReport 11:1673-1676.

Hoechstetter K, Rupp A, Meinck H-M, Weckesser D, Bornfleth H, Stippich C, Berg P, Scherg M (2000) Magnetic source imaging of tactile input shows task-independent attention effects in SII. NeuroReport 11:2461-2465. 
Hsiao SS, Lane J, Fitzgerald P (2002) Representation of orientation in the somatosensory system. Behavioural Brain Research 135:93-103.

Jenkinson M, Smith SM (2001) A global optimisation method for robust affine registration of brain images. Medical Image Analysis 5:143-156.

Johansen-Berg H, Christensen V, Woolrich M, Matthews PM (2000) Attention to touch modulates activity in both primary and secondary somatosensory areas. NeuroReport 11:1237-1241.

Johnson KO, Hsiao SS (1992) Neural mechanisms of tactual form and texture perception. Annual Review of Neuroscience 15:227-250.

Khalsa PS, Friedman RM, Srinivasan MA, Lamotte RH (1998) Encoding of shape and orientation of objects indented into the monkey fingerpad by populations of slowly and rapidly adapting mechanoreceptors. Journal of Neurophysiology 79:3238-3251.

Liu T, Larsson J, Carrasco M (2007) Feature-based attention modulates orientation-selective responses in human visual cortex. Neuron 55: 313-323.

Martinez-Trujillo JC, Treue S (2004) Feature-based attention increases the selectivity of population responses in primate visual cortex. Current Biology 14: 744-751.

Maunsell JHR, Cook EP (2002) The role of attention in visual processing. Philosophical Transactions of the Royal Society of London Series B: Biological Sciences 357: 1063-1072.

Meyer E, Ferguson SSG, Zatorre RJ, Alivisatos B, Marrett S, Evans AC, Hakim AM (1991) Attention modulates somatosensory cerebral blood flow response to vibrotactile stimulation as measured by positron emission tomography. Annals of Neurology 29: 440-443.

Mima T, Nagamine T, Nakamura K, Shibasaki H (1998) Attention modulates both primary and second somatosensory cortical activities in humans: A magnetoencephalographic study. Journal of Neurophysiology 80: 2215-2221.

Nelson AJ, Staines WR, Graham SJ, Mcllroy WE (2004) Activation in SI and SII; the influence of vibrotactile amplitude during passive and task-relevant stimulation. Cognitive Brain Research 19:174-184.

Oldfield RC (1971) The assessment and analysis of handedness: The Edinburgh inventory. Neuropsychologia 9: 97-113.

Penfield W, Rasmussen T (1950) The cerebral cortex of man: A clinical study of localization of function. New York: Macmillan.

Pons TP, Garraghty PE, Friedman DP, Mishkin M (1987) Physiological evidence for serial processing in somatosensory cortex. Science 237: 417-420.

Rossi AF, Paradiso MA (1995) Feature-specific effects of selective visual attention. Vision Research 35: 621-634.

Ruben J, Krause T, Taskin B, Blankenburg F, Moosmann M, Villringer A (2006) Subarea-specific Suppressive Interaction in the BOLD Responses to Simultaneous Finger Stimulation in Human Primary Somatosensory Cortex: Evidence for Increasing Rostral-to-caudal Convergence. Cerebral Cortex 16: 819-826.

Saenz M, Buracas GT, Boynton GM (2002) Global effects of feature-based attention in human visual cortex. Nature Neuroscience 5: 631-632.

Saenz M, Buracas GT, Boynton GM (2003) Global feature-based attention for motion and color. Vision Research 43: 629-637. 


\subsection{Exploration of feature-based somatosensory modulation of responses to orientations: A human fMRI study}

Schweisfurth MA, Schweizer R, Frahm J (2011) Functional MRI indicates consistent intra-digit topographic maps in the little but not the index finger within the human primary somatosensory cortex. Neurolmage 56: 2138-2143.

Schweisfurth MA, Katzner S, Schweizer R, Treue S (in preparation) Feature-based attentional modulation of orientation perception in somatosensation.

Schweisfurth MA, Frahm J, Schweizer R (submitted) Functional MRI reveals individual variations in the complete map of human digit phalanges in the primary somatosensory cortex.

Schweizer R, Voit D, Frahm J (2008) Finger representations in human primary somatosensory cortex as revealed by high-resolution functional MRI of tactile stimulation. Neurolmage 42: 28-35.

Serences JT, Boynton GM (2007) Feature-based attentional modulations in the absence of direct visual stimulation. Neuron 55: 301-312.

Sinclair R, Kuo J, Burton H (2000) Effects on discrimination performance of selective attention to tactile features. Somatosensory \& Motor Research 17: 145-157.

Smith SM, Jenkinson M, Woolrich MW, Beckmann CF, Behrens TEJ, Johansen-Berg H, Bannister PR, De Luca M, Drobnjak I, Flitney DE, Niazy RK, Saunders J, Vickers J, Zhang Y, De Stefano N, Brady JM, Matthews PM (2004) Advances in functional and structural MR image analysis and implementation as FSL. Neurolmage 23: S208-S219.

Staines WR, Graham SJ, Black SE, Mcllroy WE (2002) Task-relevant modulation of contralateral and ipsilateral primary somatosensory cortex and the role of a prefrontal-cortical sensory gating system. Neurolmage 15: 190-199.

Sterr A, Shen S, Zaman A, Roberts N, Szameitat A (2007) Activation of SI is modulated by attention: a random effects fMRI study using mechanical stimuli. NeuroReport 18: 607-611.

Stoppel CM, Boehler CN, Strumpf H, Heinze H-J, Noesselt T, Hopf J-M, Schoenfeld MA (2011) Feature-based attention modulates direction-selective hemodynamic activity within human MT. Human Brain Mapping 32: 2183-2192.

Treue S, Trujillo JCM (1999) Feature-based attention influences motion processing gain in macaque visual cortex. Nature 399: 575-579.

Vega-Bermudez F, Johnson KO (2001) Differences in spatial acuity between digits. Neurology 56: 1389-1391.

Yousry TA, Schmid UD, Alkadhi H, Schmidt D, Peraud A, Buettner A, Winkler P (1997) Localization of the motor hand area to a knob on the precentral gyrus. A new landmark. Brain 120: 141-157. 


\section{General discussion}

In this final section, the findings of the four studies reported in this thesis are summarized and briefly discussed in a larger context. Also, further research questions emerging from this thesis are described.

In the first part of this dissertation, the cortical representations of the dominant hand's digit area in the primary somatosensory cortex were explored through highresolution functional magnetic resonance imaging (fMRI). The results led to novel insights about their topological arrangement in Brodmann area (BA) 3b.

In Study 1 focusing on the most distal and most proximal digit parts (tip and base) of index (D2) and little (D5) finger, unexpected results were found in the layout of the map, which nevertheless could be reproduced in Study 2. This second study mapped all phalanges of all digits of the dominant hand in individual subjects and resulted in complete individual-subject digit-area maps obtained within single imaging sessions.

Across digits, the expected medial-to-lateral fingertip succession along the central sulcus from little-finger to thumb representations could be confirmed (e.g. Penfield and Boldrey, 1937; Schweizer et al., 2008). For the first time, this succession could also be demonstrated for the middle and proximal phalanges, suggesting that the digits in their entirety are represented from medial to lateral in BA 3b, as observed in non-human primates (e.g. Kaas et al., 1979).

The within-digit analysis revealed unexpected results, as no consistent intradigit maps across subjects were observed for several digits. In fact, consistent intradigit somatotopy was only found for the little finger and to some degree for the ring finger. In the thumb, middle finger, and index finger, neither a consistent direction was observed along which the digit's phalanges were represented nor were they found to be ordered from tip ( $p 1$ ) to proximal phalanx (p3) along any subject-specific axis. Our results stand in contrast to studies in anesthetized monkeys, in which ordered rostral-to-caudal p1-to-p3 representations were reported for BA 3b along with a mirror-reversed succession in BA 1 (Merzenich et al., 1978; Kaas et al., 1979; Nelson et al., 1980). In contrast, such a clear pattern could also not be shown for awake monkeys (Iwamura et al., 1983a,b). In awake monkeys, neurons responding 
to different phalanges were found to be partly mixed in BA 3b (Iwamura et al., 1983a): Most neurons responding to medial- and proximal-phalanx stimulation were found in the posterior part of $B A 3 b$, but neurons responding to the fingertips were distributed across the digit area. In BA 1, neurons coding for different intra-digit phalanges were completely mixed (Iwamura et al., 1983b) and hence mirror-imaged representations between BA $3 \mathrm{~b}$ and 1 could not be observed. These results are in reasonable agreement with our own observations.

Two earlier human fMRI studies reported across-subjects consistent intra-digit somatotopy with mirror-reversal for individual digits, one for the index finger (Sanchez-Panchuelo et al., 2012) and another for the middle finger (Blankenburg et al., 2003). However, Sanchez-Panchuelo and coworkers (2012) did not use any quantification method for exploration of the map, but employed manual delineation to define lines of mirror-reversal in the 4 out of 6 subjects for whom they reported intradigit somatotopy with mirror-reversal. For the Blankenburg et al. (2003) study exploring the middle finger, not only a low spatial resolution was used, but also could our own statistical re-calculation of their data not reproduce their significant results for the individually-based analysis. In contrast to these studies of a single digit, Study 2 of this thesis for the first time reported a complete mapping of all digits in individual subjects, paving the way for further individually-based studies on this issue. It remains controversial whether ordered and across-subjects consistent intradigit maps with mirror-reversals between $B A 3 b$ and 1 are identifiable with $f M R I$ for all digits in BA $3 b$ of individual awake humans.

As explanation for the low degree of across-subjects consistency in the somatosensory representations found here, we hypothesize that the individual hand and digit use has a strong influence on the individual-subject topography of cortical maps and hence on the presence or absence of across-subjects consistency. This conjecture is in line with suggestions by Merzenich and coworkers (1987), who also found topographic variations across subjects in the individual digit-area maps when studying a large number of anesthetized monkeys. Whether the cortical representation of the phalanges depends on individual life-long hand and digit use should be tested in future studies. It would, for example, be important to test whether a higher degree of across-subjects consistency of intra-digit maps is found for the non-dominant hand, which is less involved in precise haptic exploration and should 
therefore be less influenced by the individual digit use. In addition, it would be fruitful to explore whether the intra-digit map of individual subjects has an influence on their behavioral perception.

Another open question in mapping the primary somatosensory cortex is how to delineate Brodmann areas $3 \mathrm{a}, 3 \mathrm{~b}, 1$, and 2 in individual human subjects. In vision, the "retinotopic mapping" fMRI approach (Sereno et al., 1994, 1995) is used to define the borders between visual areas V1 to V4 along the mirror-reversal sites of one dimension (polar angle) of the visual-field representation. For the 4 out of 6 subjects for whom Sanchez-Panchuelo and co-workers (2012) observed intra-digit maps with mirror-reversals, the authors similarly defined the borders of BAs $3 \mathrm{~b}$ and 1 in the index-finger area at these mirror reversals. The results of their remaining 2 subjects did not support the presence of an ordered representation with mirrorreversals and hence did not allow for area delineation. Because the results reported in this thesis reveal individual, non-consistent intra-digit maps for several digits, these maps could not be used to delineate the BAs of the primary somatosensory cortex for the attentional Study 4 reported in the second part of this thesis. Instead, an alternative approach was developed to define the primary somatosensory cortex, taking into account anatomical atlas information as well as individual anatomical and functional information. This approach will be expanded as soon as the respective FSL toolbox allows for the recently announced sufficiently good registration of individual BAs.

The second part of this dissertation revealed insights into tactile feature-based attention, exploring it in a psychophysical Study 3 and an fMRI Study 4 using tactilely-presented orientation as relevant stimulus dimension.

Exploiting a reaction-time paradigm, Study 3 contains the first report of psychophysical correlates of tactile feature-based attention. Faster reaction times to feature-attended targets were found, not only at the spatially-attended spot for which the feature cue was informative, but also at unattended locations without feature information. Additionally, processing or perception anisotropy was found between orientations, as subjects reacted faster to the vertical compared to the horizontal target orientation. 
For Study 4, we developed an fMRI-adapted design for exploration of tactile feature-based attention in the primary and secondary somatosensory cortices. While keeping as many parameters as possible comparable to Study 3 , the design was built analogously to the fMRI study by Saenz and co-workers (2002) who reported global feature-based attention in vision. The integrated psychophysical accuracy task reproduced the processing or perception anisotropy between vertical and horizontal orientation, suggesting that the two psychophysical experiments were indeed testing a similar concept. Using fMRI, feature-based attention and anisotropy were explored at the cortical level. In prediction by the feature-similarity gain model (Treue and Martinez-Trujillo, 1999) and in analogy to the visual fMRI study by Saenz and coworkers (2002), one would expect the classical effects of global feature-based attention, i.e. a higher BOLD signal to the feature presented at the distractor location if the feature attended at the attended location is the same as the presented feature compared to a situation where the features differ. Such a classical effect of global feature-based attention could not be observed in our study neither in SI nor in SII. This is in contrast to the only published study on this issue (Forster and Eimer, 2004), which found early ERP correlates of global tactile feature-based attention for the stimulus dimensions of intensity and - only partly - frequency. In our study, a different attentional effect to features was shown: The BOLD signal at the distractor location was found to differ depending on which orientation was attended at the attended location. This finding could be related to the psychophysically-observed processing or perception anisotropy and demonstrates a far-reaching attentional effect due to attention to specific features.

Several reasons may be responsible for not observing the classical effects of feature-based attention in this first fMRI study, as discussed in detail within Study 4. Due to the persuading psychophysical results, the present fMRI results should not be taken as an indication for the non-existence of feature-based attentional correlates in SI and SII. Instead, the fMRI experiment is considered to be very fruitful in paving the way for a large number of further relevant studies. If adhering to the stimulus dimension of orientation, it seems crucial to devote more research to the exploration of tactile anisotropy in orientation processing or perception. This would not only increase the body of knowledge for this controversial topic, but might also ensure that feature-based effects are not obscured by anisotropic processing or perception. 
Behavioral and cortical correlates of feature-based attention should also be explored for other tactile stimulus dimensions important for everyday haptics, such as roughness, motion direction, and frequency. Together, such work is expected to build up a more complete picture of feature-based attention in the somatosensory system.

In conclusion, this dissertation addressed several important and controversial issues concerning the human somatosensory system, mainly focusing on digit-area somatotopy and feature-based attention. It revealed novel insights into both topics and paves the way for subsequent research. This dissertation advances critical issues in somatosensory research and emphasizes that more attention should be devoted to the rather special sense of touch. 


\section{Bibliography}

Amaral DG (2000) 18. The functional organization of perception and motion. In: Principles of Neural Science., 4th Edition Edition (Kandel ES, JH; Jessell, TM; Siegelbaum, SA; Hudspeth, AJ, ed), pp 337-348. New York: McGraw-Hill.

Blankenburg F, Ruben J, Meyer R, Schwiemann J, Villringer A (2003) Evidence for a rostral-to-caudal somatotopic organization in human primary somatosensory cortex with mirror-reversal in areas $3 b$ and 1. Cerebral Cortex 13: 987-993.

Braun C, Haug M, Wiech K, Birbaumer N, Elbert T, Roberts LE (2002) Functional organization of primary somatosensory cortex depends on the focus of attention. Neurolmage 17: 1451-1458.

Brodmann K (1909) Vergleichende Lokalisationslehre der Grosshirnrinde. Leipzig(DE): Barth.

Buchner H, Richrath P, Grünholz J, Noppeney U, Waberski TD, Gobbele R, Willmes K, Treede RD (2000) Differential effects of pain and spatial attention on digit representation in the human primary somatosensory cortex. NeuroReport 11: 1289-1293.

Burton H, Abend NS, MacLeod AMK, Sinclair RJ, Snyder AZ, Raichle ME (1999) Tactile attention tasks enhance activation in somatosensory regions of parietal cortex: A positron emission tomography study. Cerebral Cortex 9: 662-674.

Burton H, Sinclair RJ, McLaren DG (2008) Cortical network for vibrotactile attention: A fMRI study. Human Brain Mapping 29: 207-221.

Chica AB, Sanabria D, Lupianez J, Spence C (2007) Comparing intramodal and crossmodal cuing in the endogenous orienting of spatial attention. Experimental Brain Research 179: 353-364.

Corbetta M, Miezin FM, Dobmeyer S, Shulman GL, Petersen SE (1990) Attentional modulation of neural processing of shape, color, and velocity in humans. Science (New York, NY) 248: 1556-1559.

Desmedt JE, Robertson D (1977) Differential enhancement of early and late components of the cerebral somatosensory evoked potentials during forced-paced cognitive tasks in man. The Journal of Physiology 271: 761-782.

Eimer M, Forster B (2003) The spatial distribution of attentional selectivity in touch: evidence from somatosensory ERP components. Clinical Neurophysiology 114: 1298-1306.

Eskenasy A-CC, Clarke S (2000) Hierarchy within human SI: supporting data from cytochrome oxidase, acetylcholinesterase and NADPH-diaphorase staining patterns. Somatosensory \& Motor Research 17: 123-132.

Forster B, Eimer M (2004) The attentional selection of spatial and non-spatial attributes in touch: ERP evidence for parallel and independent processes. Biological Psychology 66: 1-20.

Forster B, Eimer M (2005) Covert attention in touch: Behavioral and ERP evidence for costs and benefits. Psychophysiology 42: 171-179.

Gardner EP (1988) Somatosensory cortical mechanisms of feature detection in tactile and kinesthetic discrimination. Canadian Journal of Physiology and Pharmacology 66: 439-454.

Gardner EP, Kandel ER (2000) 23. Touch. In: Principles of Neural Science., 4th Edition Edition (Kandel ES, JH; Jessell, TM; Siegelbaum, SA; Hudspeth, AJ, ed), pp 498-529. New York: McGraw-Hill.

Gardner E, Martin J, Jessell T (2000) 22. The bodily senses. In: Principles of Neural Science. (Kandel ES, JH; Jessell, TM; Siegelbaum, SA; Hudspeth, AJ, ed), pp 430-449. New York: McGrawHill. 
Gardner EP (2010) Touch. In: Encyclopedia of Life Sciences. Chichester: John Wiley \& Sons, Ltd.

Gardner EP, Johnson K (2012) 23. Touch. In: Principles of Neural Science., 5th Edition Edition (Kandel ES, JH; Jessell, TM; Siegelbaum, SA; Hudspeth, AJ, ed), pp 498-529. New York: McGraw-Hill.

Garraghty PE, Florence SL, Kaas JH (1990) Ablations of areas 3a and 3b of monkey somatosensory cortex abolish cutaneous responsivity in area 1. Brain Research 528: 165-169.

Geyer S, Schleicher A, Zilles K (1999) Areas 3a, 3b, and 1 of Human Primary Somatosensory Cortex: 1. Microstructural Organization and Interindividual Variability. Neurolmage 10: 63-83.

Geyer S, Schormann T, Mohlberg H, Zilles K (2000) Areas 3a, 3b and 1 of Human primary Somatosensory cortex, 2. Spatial normalisation to standard anatomical space. Neurolmage 11: 684-696.

Gillmeister H, Adler J, Forster B (2010) Object-guided spatial attention in touch: Holding the same object with both hands delays attentional selection. Journal of Cognitive Neuroscience 22: 931-942.

Gillmeister H, Cantarella S, Gheorghiu Al, Adler J (2013) Object-guided spatial selection in touch without concurrent changes in the perceived location of the hands. Experimental Psychology 60: 64-70.

Hämäläinen H, Hiltunen J, Titievskaja I (2000) fMRI activations of SI and SII cortices during tactile stimulation depend on attention. NeuroReport 11: 1673-1676.

Hoechstetter K, Rupp A, Meinck H-M, Weckesser D, Bornfleth H, Stippich C, Berg P, Scherg M (2000) Magnetic source imaging of tactile input shows task-independent attention effects in SII. NeuroReport 11: 2461-2465.

Hsiao SS, O'Shaughnessy DM, Johnson KO (1993) Effects of selective attention on spatial form processing in monkey primary and secondary somatosensory cortex. Journal of Neurophysiology 70: 444-447.

Hyvärinen A, Oja E (2000) Independent component analysis: algorithms and applications. Neural Networks 13: 411-430.

Iwamura Y, Tanaka M, Sakamoto M, Hikosaka O (1983a) Functional subdivisions representing different finger regions in area 3 of the first somatosensory cortex of the conscious monkey. Experimental Brain Research 51: 315-326.

Iwamura Y, Tanaka M, Sakamoto M, Hikosaka O (1983b) Converging patterns of finger representation and complex response properties of neurons in area 1 of the first somatosensory cortex of the conscious monkey. Experimental Brain Research 51: 327-337.

Johansen-Berg H, Christensen V, Woolrich M, Matthews PM (2000) Attention to touch modulates activity in both primary and secondary somatosensory areas. NeuroReport 11: 1237-1241.

Johansson RS, Vallbo AB (1979) Tactile sensibility in the human hand: relative and absolute densities of four types of mechanoreceptive units in glabrous skin. The Journal of Physiology 286: 283-300.

Jones EG, Friedman DP (1982) Projection pattern of functional components of thalamic ventrobasal complex on monkey somatosensory cortex. Journal of Neurophysiology 48: 521-544.

Kaas JH, Nelson RJ, Sur M, Lin CS, Merzenich MM (1979) Multiple representations of the body within the primary somatosensory cortex of primates. Science 204: 521-523.

Martinez-Trujillo JC, Treue S (2004) Feature-based attention increases the selectivity of population responses in primate visual cortex. Current Biology 14: 744-751. 
Martuzzi R, van der Zwaag W, Farthouat J, Gruetter R, Blanke O (in press) Human finger somatotopy in areas $3 \mathrm{~b}, 1$, and 2: A $7 \mathrm{~T}$ fMRI study using a natural stimulus. Human Brain Mapping (in press).

Merzenich MM, Kaas JH, Sur M, Lin C-S (1978) Double representation of the body surface within cytoarchitectonic area $3 \mathrm{~b}$ and 1 in $\mathrm{SI}$ in the owl monkey (aotus trivirgatus). The Journal of Comparative Neurology 181: 41-73.

Merzenich MM, Nelson RJ, Kaas JH, Stryker MP, Jenkins WM, Zook JM, Cynader MS, Schoppmann A (1987) Variability in hand surface representations in areas $3 \mathrm{~b}$ and 1 in adult owl and squirrel monkeys. The Journal of Comparative Neurology 258: 281-296.

Mountcastle VB, LaMotte RH, Carli G (1972) Detection thresholds for stimuli in humans and monkeys: comparison with threshold events in mechanoreceptive afferent nerve fibers innervating the monkey hand. Journal of Neurophysiology 35: 122-136.

Mulckhuyse M, Theeuwes J (2010) Unconscious attentional orienting to exogenous cues: A review of the literature. Acta Psychologica 134: 299-309.

Nelson RJ, Sur M, Felleman DJ, Kaas JH (1980) Representations of the body surface in postcentral parietal cortex of Macaca fascicularis. The Journal of Comparative Neurology 192: 611-643.

Nelson AJ, Staines WR, Graham SJ, Mcllroy WE (2004) Activation in SI and SII; the influence of vibrotactile amplitude during passive and task-relevant stimulation. Cognitive Brain Research 19: 174-184.

Nelson AJ, Chen R (2008) Digit somatotopy within cortical areas of the postcentral gyrus in humans. Cerebral Cortex 18: 2341-2351.

Noppeney U, Waberski TD, Gobbele R, Buchner H (1999) Spatial attention modulates the cortical somatosensory representation of the digits in humans. NeuroReport 10: 3137-3141.

O'Craven KM, Downing PE, Kanwisher N (1999) fMRI evidence for objects as the units of attentional selection. Nature 401: 584-587.

Overduin SA, Servos P (2004) Distributed digit somatotopy in primary somatosensory cortex. Neurolmage 23: 462-472.

Penfield W, Boldrey E (1937) Somatic motor and sensory representation in the cerebral cortex of man as studied by electrical stimulation. Brain 60: 389-443.

Penfield W, Rasmussen T (1950) The cerebral cortex of man: A clinical study of localization of function. New York: Macmillan.

Pons TP, Garraghty PE, Friedman DP, Mishkin M (1987) Physiological evidence for serial processing in somatosensory cortex. Science 237: 417-420.

Posner MI (1978) Chronometric explorations of mind: Oxford, England: Lawrence Erlbaum.

Posner MI (1980) Orienting of attention. Quarterly Journal of Experimental Psychology 32: 3-25.

Rossi AF, Paradiso MA (1995) Feature-specific effects of selective visual attention. Vision Research 35: 621-634.

Saenz M, Buracas GT, Boynton GM (2002) Global effects of feature-based attention in human visual cortex. Nature Neuroscience 5: 631-632.

Saenz M, Buracas GT, Boynton GM (2003) Global feature-based attention for motion and color. Vision Research 43: 629-637. 
Sanchez-Panchuelo RM, Francis S, Bowtell R, Schluppeck D (2010) Mapping human somatosensory cortex in individual subjects with 7T functional MRI. Journal of Neurophysiology 103: 2544-2556.

Sanchez-Panchuelo RM, Besle J, Beckett A, Bowtell R, Schluppeck D, Francis S (2012) Within-digit functional parcellation of Brodmann areas of the human primary somatosensory cortex using functional magnetic resonance imaging at 7 Tesla. The Journal of Neuroscience 32: 15815-15822.

Sathian K, Burton H (1991) The role of spatially selective attention in the tactile perception of texture. Perception and Psychophysics 50: 237-248.

Schweisfurth MA, Schweizer R, Frahm J (2011) Functional MRI indicates consistent intra-digit topographic maps in the little but not the index finger within the human primary somatosensory cortex. Neurolmage 56: 2138-2143.

Schweizer R, Voit D, Frahm J (2008) Finger representations in human primary somatosensory cortex as revealed by high-resolution functional MRI of tactile stimulation. Neurolmage 42: 28-35.

Simons DJ, Chabris CF (1999) Gorillas in our midst: Sustained inattentional blindness for dynamic events. Perception 28: 1059-1074.

Sinclair R, Kuo J, Burton H (2000) Effects on discrimination performance of selective attention to tactile features. Somatosensory \& Motor Research 17: 145-157.

Spence C, Pavani F, Driver J (2000) Crossmodal links between vision and touch in covert endogenous spatial attention. Journal of Experimental Psychology: Human Perception and Performance 26: 1298-1319.

Sterr A, Shen S, Zaman A, Roberts N, Szameitat A (2007) Activation of SI is modulated by attention: a random effects fMRI study using mechanical stimuli. NeuroReport 18: 607-611.

Stoppel CM, Boehler CN, Strumpf H, Heinze H-J, Noesselt T, Hopf J-M, Schoenfeld MA (2011) Feature-based attention modulates direction-selective hemodynamic activity within human MT. Human Brain Mapping 32: 2183-2192.

Sur M, Merzenich MM, Kaas JH (1980) Magnification, receptive-field area, and "hypercolumn" size in areas $3 \mathrm{~b}$ and 1 of somatosensory cortex in owl monkeys. Journal of Neurophysiology 44: 295-311.

Treisman AM, Gelade G (1980) A feature-integration theory of attention. Cognitive Psychology 12: 97-136.

Treue S, Trujillo JCM (1999) Feature-based attention influences motion processing gain in macaque visual cortex. Nature 399: 575-579.

Treue S (2001) Neural correlates of attention in primate visual cortex. Trends in Neurosciences 24: 295-300.

Treue S (2003) Visual attention: the where, what, how and why of saliency. Current Opinion in Neurobiology 13: 428-432.

Treue S, Martinez-Trujillo JC (2007) Attending to features inside and outside the spotlight of attention. Neuron 55: 174-176.

van Ede F, de Lange FP, Maris E (2012) Attentional cues affect accuracy and reaction time via different cognitive and neural processes. The Journal of Neuroscience 32: 10408-10412.

Vogt C, Vogt O (1919) Allgemeinere Ergebnisse unserer Hirnforschung. Journal fuer Psychologie und Neurologie 25: 279-462. 
Bibliography 


\section{Abbreviations}

ACPC: $\quad$ anterior commissure - posterior commissure

ANOVA: analysis of variance

BA: $\quad$ Brodmann area

BOLD: $\quad$ blood oxygenation level dependent

COM: $\quad$ center of mass

D1: $\quad$ thumb

D2: $\quad$ index finger

D3: $\quad$ middle finger

D4: ring finger

D5: little finger

ERA: event-related average

ERP: $\quad$ event-related potential

EPI: $\quad$ echo-planar imaging

FDR: $\quad$ false discovery rate

FLASH: fast low angle shot

FLIRT: $\quad$ FMRIB's linear image registration tool

fMRI: functional magnetic resonance imaging

FNIRT: $\quad$ FMRIB's nonlinear image registration tool

FSL: $\quad$ FMRIB Software Library

GLM: $\quad$ general linear model

LATER: linear approach to threshold with ergodic rate 
MNI: $\quad$ Montreal Neurological Institute

MPRAGE: magnetization-prepared rapid gradient echo

MRI: $\quad$ magnetic resonance imaging

p1: $\quad$ first phalanx (fingertip)

p2: $\quad$ second phalanx

p3: $\quad$ third phalanx

p4: $\quad$ digit base (palmar skin over caput of metacarpal bone radial to digit)

RM ANOVA: repeated-measures analysis of variance

ROI: $\quad$ region of interest

RT: $\quad$ reaction time

SD: $\quad$ standard deviation

SEM: $\quad$ standard error of the mean

SI: $\quad$ primary somatosensory cortex

SII: $\quad$ secondary somatosensory cortex

TA: acquisition time

TE: $\quad$ echo time

TR: repetition time 


\section{Curriculum Vitae}

\section{Personal details}

\begin{tabular}{ll}
\hline Full name: & Meike Annika Schweisfurth \\
Place of birth: & Langenfeld (Rheinland), Germany \\
Date of birth: & $19^{\text {th }}$ of October 1984
\end{tabular}

Scientific Education

Since 2009

Graduate student within International Max Planck Research School (IMPRS) for Neurosciences within the Goettingen Graduate School for Neursciences, Biophysics, and Molecular Biosciences (GGNB), combinedly at

- Biomedizinische NMR Forschungs GmbH (BiomedNMR), Max Planck Institute for Biophysical Chemistry, Göttingen, Germany, and

- Cognitive Neuroscience Laboratory (CNL), German Primate Center, Göttingen, Germany

Advisors: Dr. Renate Schweizer

Prof. Dr. Jens Frahm

Prof. Dr. Stefan Treue

$2008-2009$

Neuroscience master education at Göttingen University in the IMPRS for Neurosciences

Examination: Grade "very good"

$2007-2008$

Master of Advanced Studies (former Certificate of Advanced Studies of Mathematics) at University of Cambridge, England

Examination: Grade "distinction"

$2004-2007$

Studies of mathematics and minor subject theoretical physics at Georg-August-Universität Göttingen, Germany Vordiplom: Grade "sehr gut" (very good)

$1997-2004$

Albert-Einstein-Gymnasium (Buchholz in der Nordheide) Abitur (Graduation): Grade "1.0” (very good) 


\section{Laboratory experience}

2009

Lab rotation projects within the IMPRS master program:

- "The role of CG14921 in Drosophila hearing", Dept. of Cellular Neurobiology, Georg-August-Universität Gottingen

Advisor: Prof. Dr. Martin Göpfert

- "Which frequencies can neurons track? Phase locking in mean and variance modulation", Nonlinear

Dynamics, Max Planck Institute for Dynamics and SelfOrganisation

Advisor: Prof. Dr. Fred Wolf

- "From the basics of NMR to studying imaginary hand movements using the game BRAIN PONG", Biomedizinische NMR Forschungs $\mathrm{GmbH}$, Max Planck Institute for Biophysical Chemistry Advisor: Prof. Dr. Jens Frahm

Teaching experience 2012

2012

$2010-2012$

2011

$2006-2007$
Two-months supervision of three lab rotation students of the Graduate Program Neurosciences, at BiomedNMR

Two-weeks supervision of a school student

Lecture on $\mathrm{fMRI}$ within the master education of the Graduate Program Neurosciences

Six-weeks supervision of a bachelor student, at CNL

Tutorial on Analytic Geometry and Linear Algebra at the Institute for Mathematics, Goettingen

Courses and workshops
2011
Summer School in Computational Sensory-Motor
Neuroscience, Kingston, Canada
2010
FSL and Freesurfer Course, Heidelberg, Germany
2010
MATLAB course, Göttingen, Germany
2010
Course in Transcranial Magnetic and Electrical Stimulation
(TMS / tDCS / tACS / tRNS), Göttingen, Germany
Psychophysics course, Göttingen, Germany 


\section{Conferences and contributions}

Planned for $2013 \quad$ Human Brain Mapping Conference, Seattle, USA

2012

Society for Neuroscience Meeting, New Orleans, USA Poster: $\quad$ Somatosensory feature-based attention observed in human reaction-time experiments.

2012

Human Brain Mapping Conference, Beijing, China Poster: $\quad$ Novel analysis approach to test for intra-digit somatotopy in $\mathrm{fMRI}$ data of human BA3b.

2011

Organization of and participation in Neurizons conference, Göttingen, Germany

Poster: $\quad$ Functional MRI indicates consistent intradigit topographic maps in the little but not the index finger within the human primary somatosensory cortex.

2011

Human Brain Mapping Conference, Québec, Canada

Poster: $\quad$ Functional MRI indicates consistent intradigit topographic maps in the little but not the index finger within the human primary somatosensory cortex.

Human Brain Mapping Conference, Barcelona, Spain

\section{Publications}

Submitted

2011
Meike A. Schweisfurth, Jens Frahm, Renate Schweizer Functional MRI reveals individual variations in the complete map of human digit phalanges in the primary somatosensory cortex.

Meike A. Schweisfurth, Renate Schweizer, Jens Frahm Functional MRI indicates consistent intra-digit topographic maps in the little but not the index finger within the human primary somatosensory cortex

Neurolmage (2011), volume 56, issue 4, pp. 2138-2143. 


\section{Scholarships}

\begin{tabular}{ll}
\hline $2009-2013$ & $\begin{array}{l}\text { Neurosenses Scholarship } \\
\text { Scholarship of the Excellence Foundation for the Promotion } \\
\text { of Max Planck Society }\end{array}$ \\
$2008-2009$ & DAAD-Scholarship (German Academic Exchange Service) \\
$2007-2008$ & Allianz-Scholarship \\
$2007-2008$ & Cambridge European Trust Scholarship \\
$2005-2009$ & Scholarship of Studienstiftung des deutschen Volkes \\
Since 2004 & Online-Scholarship at e-fellows.net
\end{tabular}

\section{Languages}

\section{German:}

English:

Spanish:

Latin:

French:

Italian:
Mother tongue

TOEFL-iBT (2007): 108/120 points, one-year stay in Cambridge (England)

Diploma Intermedio de Español in 2001, four month stay at Colegio Internacional Lope de Vega (Benidorm, Spain)

"Großes Latinum"

Four-year school education

Basic level, self-study

\section{Hobbies}

Travelling, choirs, guitar and piano, tennis, skiing 